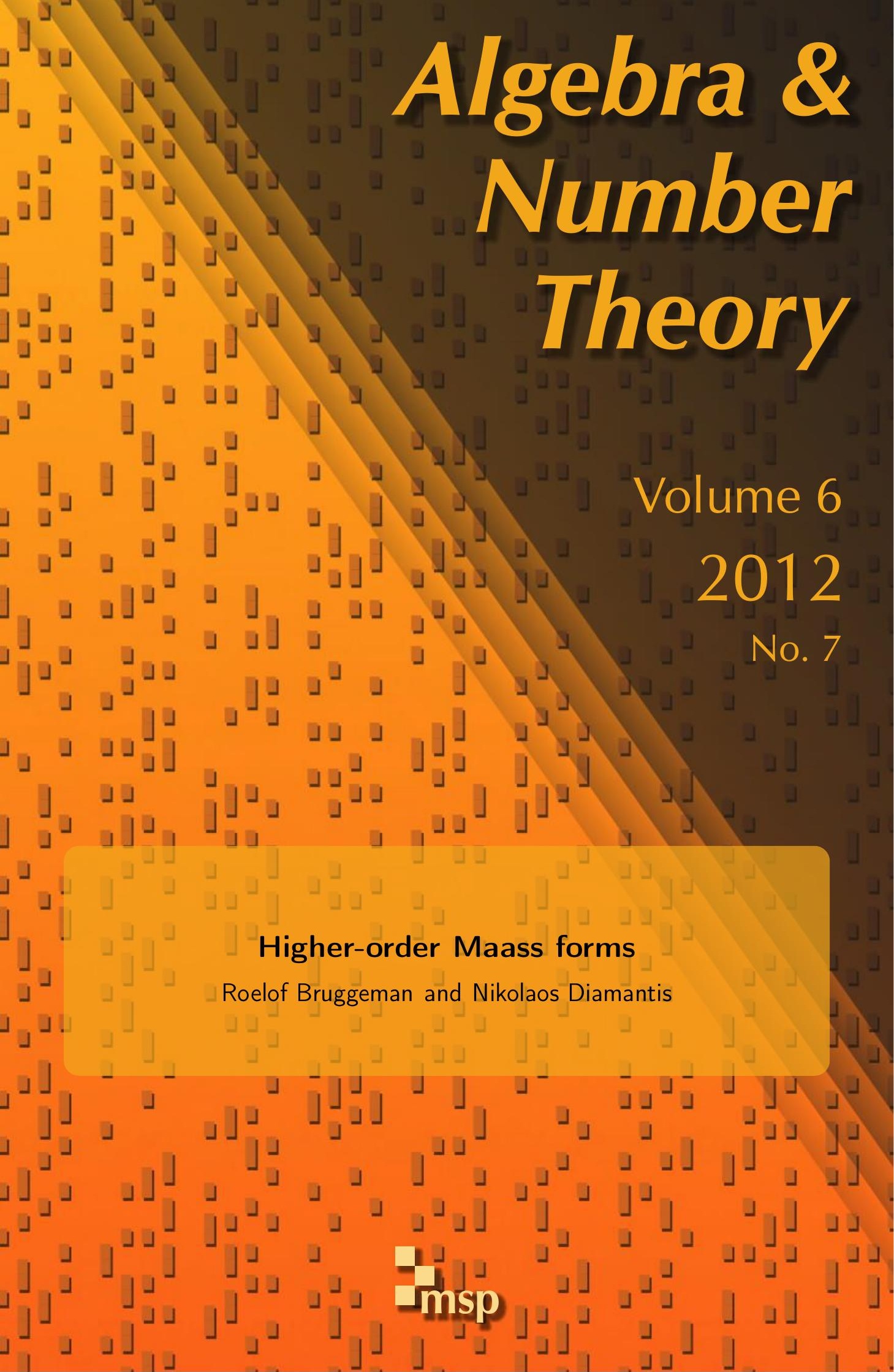




\title{
Higher-order Maass forms
}

\author{
Roelof Bruggeman and Nikolaos Diamantis
}

The spaces of Maass forms of even weight and of arbitrary order are studied. It is shown that, if we allow exponential growth at the cusps, these spaces are as large as algebraic restrictions allow. These results also apply to higher-order holomorphic forms of even weight.

\section{Introduction}

Occasionally, invariants of classical holomorphic modular forms can be studied effectively by means of generating functions that are nonanalytic. An example is the Eisenstein series modified with modular symbols. It is defined by

$$
E^{*}(z, s)=\sum_{\gamma \in \Gamma_{\infty} \backslash \Gamma_{0}(N)}\langle f, \gamma\rangle \operatorname{Im}(\gamma z)^{s},
$$

where $\Gamma_{\infty}$ is the subgroup of translations of the congruence group $\Gamma_{0}(N), f$ is a weight- 2 newform, and $\langle f, \gamma\rangle$ denotes its modular symbol $-2 \pi i \int_{\infty}^{\gamma \infty} f(w) d w$. The function $E^{*}(-, s)$ is not analytic, but rather an eigenfunction of the Laplace operator $-y^{2} \partial_{y}^{2}-y^{2} \partial_{x}^{2}$ with eigenvalue $s-s^{2}$. Its study has led to important results about modular symbols, such as the proof that the suitably normalized modular symbols follow the normal distribution [Petridis and Risager 2004]. A crucial feature of $E^{*}(-, s)$ is that it is not invariant under the action of $\Gamma_{0}(N)$, but instead it is $\Gamma_{0}(N)$-invariant of order 2 .

This function was one of the motivating examples for the systematic study of invariants of order $q$ for a $\Gamma$-module $V$ (over $\mathbb{C}$ ), that is, $v \in V$ satisfying

$$
v \mid\left(1-\gamma_{1}\right)\left(1-\gamma_{2}\right) \ldots\left(1-\gamma_{q}\right)=0
$$

for all $\gamma_{1}, \ldots, \gamma_{q} \in \Gamma$. The usual space of invariants $V^{\Gamma}$ consists of the invariants of order 1. Also, $E^{*}(-, s)$ is invariant of order 2 in terms of the right regular representation of $\Gamma_{0}(N)$ on the space of functions on $\mathfrak{H}$ that are eigenfunctions of the Laplacian.

MSC2010: primary 11F12; secondary $11 \mathrm{~F} 37,11 \mathrm{~F} 99,30 \mathrm{~F} 35$.

Keywords: higher-order automorphic forms, Maass forms. 
Although higher-order invariants have been classified in several cases and from various perspectives [Chinta et al. 2002; Diamantis and O'Sullivan 2008; Diamantis and Sim 2008; Deitmar 2008; 2009], the real-analytic case to which the important function $E^{*}(-, s)$ belongs has not been fully addressed up to now. This is perhaps not surprising, given that such functions can contain very rich and complex information, as the example of $E^{*}(-, s)$ shows. The resolution of the problem of classification of higher-order Maass forms is the subject of the present paper. This task includes various aspects that are often automatic in the classification of higher-order invariants of other spaces, and it requires new techniques from the theory of families of automorphic forms and perturbation theory. The most important of these aspects, all of independent interest, are:

Firstly, while in the setting of [Diamantis and O'Sullivan 2008] the "size" of the space of higher-order invariants is expressed by its (finite) dimension, in the present case a different concept is required because the relevant spaces are, in general, infinitely dimensional. This concept is maximal perturbability. Specifically, let $V^{\Gamma, q}$ be the space of invariants of order $q$ of a general group $\Gamma$ and a $\Gamma$-module $V$. If $\Gamma$ is finitely generated, there is an exact sequence

$$
0 \longrightarrow V^{\Gamma, q} \longrightarrow V^{\Gamma, q+1} \longrightarrow\left(V^{\Gamma}\right)^{n(\Gamma, q)},
$$

where the natural number $n(\Gamma, q)$ is determined by the structure of $\Gamma$. If for every $q \geq 1$ the map in (1-3) is surjective, we call the $\Gamma$-module $V$ maximally perturbable. Our choice of the word "perturbable" stems from the fact that derivatives of families of automorphic form can lead to higher-order automorphic forms; see Section 4C3. A derivative of order $q$ leads to an invariant $v$ of order $q+1$ with the special property that there is an invariant $w \in V^{\Gamma}$ such that for all choices of $\gamma_{1}, \ldots, \gamma_{q} \in \Gamma$, there is $\mu\left(\gamma_{1}, \ldots, \gamma_{q}\right) \in \mathbb{C}$ with

$$
v \mid\left(\gamma_{1}-1\right) \ldots\left(\gamma_{q}-1\right)=\mu\left(\gamma_{1}, \ldots, \gamma_{q}\right) w .
$$

We call $v$ a perturbation of $w$. On the other hand, the existence of perturbations in which $\mu\left(\gamma_{1}, \ldots, \gamma_{q}\right)$ is not symmetric in the $\gamma_{j}$ implies that not all perturbations come from differentiation of families.

Secondly, the choice of the space $V$ is a nontrivial matter. The obvious choice of functions with polynomial growth at the cusps leads to modules that are not maximally perturbable; we have to allow exponential growth. See Theorems 4.3 and 4.2.

Finally, Fourier expansions are not straightforward extensions of their classical counterparts either, because of the lack of usual invariance. Indeed, Section 7 is devoted to the development of a higher-order version of the theory of Fourier terms and expansions.

Our results imply that if $V$ is maximally perturbable, an invariant in $V^{\Gamma}$ has 
"many" perturbations. At the same time, the complexity of the constructions partly explains why it is surprisingly difficult, even in simple situations, to explicitly construct perturbations of a given invariant. In Section 4C we investigate this for the simplest example of an automorphic form that one can think of, the constant function 1. We succeed in giving a basis for all holomorphic perturbations and for all harmonic perturbations of 1 up to order 3 .

As in the classical case, the general Maass setting discussed so far includes holomorphic higher-order invariants. Important examples of the latter emerge from problems in the theory of classical modular forms: in [Goldfeld 1995] and [Diamantis 1999], certain "period integrals" are associated to derivatives of $L$ functions of weight- 2 cusp forms in a way analogous to the link between values of $L$-functions and modular integrals [Manin 1972]. Specifically, let $f$ be a newform of weight 2 for $\Gamma_{0}(N)$, and let $L_{f}(s)$ be its $L$-function. If $L_{f}(1)=0$, then $L_{f}^{\prime}(1)$ can be written as a linear combination of integrals of the form

$$
\int_{0}^{\gamma(0)} f(z) u(z) d z, \quad \gamma \in \Gamma_{0}(N),
$$

plus some "lower-order terms". Here $u(z):=\log \eta(z)+\log \eta(N z)$, where $\eta$ is the Dedekind eta function. The differential $f(z) u(z) d z$ is not $\Gamma_{0}(N)$-invariant. It does satisfy a transformation law which is reminiscent of (1-2) with $q=2$, but is not quite $\Gamma_{0}(N)$-invariant, as it has an additional term. If it were invariant, the value of the derivative at 1 would be expressed as the value of the actual $L$-function of second-order $\Gamma_{0}(N)$ at 1 . That could be advantageous for the study of $L_{f}^{\prime}(1)$ in terms of the outstanding conjectures (Beilinson, Birch-Swinnerton-Dyer, etc.), especially since there is now evidence that a motivic structure underlies higher-order forms (see [Diamantis and Sreekantan 2006; Sreekantan 2009]).

Here we show that it is indeed possible to obtain a second-order $\Gamma_{0}(N)$-invariant function from $u(z)$, provided we move to a different domain. This domain is the universal covering group $\tilde{G}$, defined in detail in Section $5 \mathrm{~A}$. It was convenient and more general to carry out the entire study (that is, of both Maass and holomorphic higher-order invariants) on $\tilde{G}$.

The main theorems of the paper (Theorems 6.5 and 6.8) classify the spaces into which we incorporate the above two important examples $\left(E^{*}(-, s)\right.$ and $\left.u(z)\right)$, in the sense that we show that these spaces are as large as they can a priori be.

\section{Structure of the paper}

In Section 3, we first discuss higher-order invariants for general groups and modules. Here we define the property of maximal perturbability.

In Section 4, Maass forms on $\mathfrak{H}$ (both general and holomorphic) are defined, and the first two main theorems of the paper (4.2 and 4.3) are stated. Since 
$\operatorname{hom}\left(\Gamma_{\bmod }, \mathbb{C}\right)=\{0\}$, there are no higher-order invariants for the full modular group $\Gamma_{\bmod }=\mathrm{PSL}_{2}(\mathbb{Z})$. For examples, we go to a subgroup of order 6 and show that some well-known functions lead to higher-order invariants. To get all perturbations of 1 up to order 3, we have to look also at less known functions.

In Section 5, the universal covering group $\tilde{G}$ is introduced, and basic facts about $\tilde{G}$ are given. Section 6 starts with the interpretation of $\log \eta$ as a second-order form on the universal covering group $\tilde{G}$ for the inverse image $\tilde{\Gamma}_{\text {mod }}$ of the modular group in $\tilde{G}$. We define Maass forms on the universal covering group in Section 6, and, in Theorems 6.5 and 6.8, we state the counterparts of Theorems 4.2 and 4.3 for forms on the universal covering group. The section concludes with concrete examples of low-order forms for the discrete subgroup $\tilde{\Gamma}_{\text {mod }}$ of $\tilde{G}$.

Section 7 is of independent interest. A theory of Fourier expansions for higherorder forms is developed. Working on the universal covering group, we have to handle invariants for the commutative group generated by a parabolic element and the center of $\tilde{G}$. So all perturbations we meet are commutative.

The proof of Theorems 6.5 and 6.8 is the content of Section 8. We start with the maximal perturbability of the space of all functions on $\tilde{G}$. Step by step, we impose more and more analytic restrictions, such as smoothness, growth behavior at cusps, and the behavior under certain differential operators. For each step, we show that maximal perturbability is preserved. For several steps, this involves induction with respect to the order. At the end, we complete the proof of our main results by an application of spectral theory.

\section{Higher-order invariants}

In this section, we discuss higher-order invariants in general and then specialize their study to discrete cofinite subgroups $\Gamma \subset \mathrm{PSL}_{2}(\mathbb{R})$. We introduce the concept of a maximally perturbable $\Gamma$-module to make precise the statement that there are as many higher-order invariants of a given type as one can expect.

3A. Higher-order invariants on general groups. The concept of higher-order invariant functions on the upper half-plane is a special case of the concept of higher-order invariants for any group $\Gamma$ and any $\Gamma$-module $V$. We work with right $\Gamma$-modules, and write the action as $v \mapsto v \mid \gamma$. It should be clear from the context when we refer to this general meaning of $\mid$ and when to the more narrow meaning given in the Introduction. We define the higher-order invariants inductively:

$$
\begin{gathered}
V^{\Gamma, 1}=V^{\Gamma}=\{v \in V: v \mid \gamma=v \text { for all } \gamma \in \Gamma\}, \\
V^{\Gamma, q+1}=\left\{v \in V: v \mid(\gamma-1) \in V^{\Gamma, q} \text { for all } \gamma \in \Gamma\right\} .
\end{gathered}
$$

We set $V^{\Gamma, 0}=\{0\}$.

Now let $\Gamma$ be finitely generated and let $I$ be the augmentation ideal in the group 
ring $\mathbb{C}[\Gamma]$, generated by $\gamma-1$ with $\gamma \in \Gamma \backslash\{1\}$. A fundamental role in this paper will be played by the map

$$
\mathrm{m}_{q}: V^{\Gamma, q+1} \rightarrow \operatorname{hom}_{\mathbb{C}[\Gamma]}\left(I^{q+1} \backslash I^{q}, V^{\Gamma}\right) .
$$

To define it, we first quote from [Deitmar 2009] (before Proposition 1.2):

$$
V^{\Gamma, q} \cong \operatorname{hom}_{\mathbb{C}[\Gamma]}\left(I^{q} \backslash \mathbb{C}[\Gamma], V\right) .
$$

The isomorphism is induced by the map $\varphi \mapsto \varphi(1)$ from $\operatorname{hom}_{\mathbb{C}[\Gamma]}(\mathbb{C}[\Gamma], V)$ to $V$. Next, we note that $I^{q+1} \backslash I^{q}$ is generated by

$$
I^{q+1}+\left(\gamma_{1}-1\right) \ldots\left(\gamma_{q}-1\right),
$$

with $\gamma_{i} \in \Gamma$. To each $v \in V^{\Gamma, q+1}$ we associate the map on $I^{q+1} \backslash I^{q}$ sending this element to $v \mid\left(\gamma_{1}-1\right) \ldots\left(\gamma_{q}-1\right)$. This map is well-defined because

$$
v \mid\left(\gamma_{1}-1\right) \ldots\left(\gamma_{q+1}-1\right)=0 .
$$

In this way, we obtain a map $\mathrm{m}_{q}$ from $V^{\Gamma, q+1}$ to

$$
\operatorname{hom}_{\mathbb{C}[\Gamma]}\left(I^{q+1} \backslash I^{q}, V\right) \cong \operatorname{hom}_{\mathbb{C}[\Gamma]}\left(I^{q+1} \backslash I^{q}, V^{\Gamma}\right)
$$

(since the action induced on $I^{q+1} \backslash I^{q}$ by the operation of $\Gamma$ is trivial). It is easy to see that the kernel of $\mathrm{m}_{q}$ is $V^{\Gamma, q}$, and thus we obtain the exact sequence

$$
0 \longrightarrow V^{\Gamma, q} \longrightarrow V^{\Gamma, q+1} \stackrel{\mathrm{m}_{q}}{\longrightarrow} \operatorname{hom}_{\mathbb{C}[\Gamma]}\left(I^{q+1} \backslash I^{q}, V\right) .
$$

The map $\mathrm{m}_{q}$ may or may not be surjective, and we will interpret the phrase "as large as possible" as surjectivity of $\mathrm{m}_{q}$ for all $q \in \mathbb{N}$.

Definition 3.1. Let $\Gamma$ be a finitely generated group. We will call a $\Gamma$-module $V$ maximally perturbable if the linear map $\mathrm{m}_{q}: V^{\Gamma, q+1} \rightarrow \operatorname{hom}_{\mathbb{C}[\Gamma]}\left(I^{q+1} \backslash I^{q}, V^{\Gamma}\right)$ is surjective for all $q \geq 1$.

A reformulation of this definition, which is occasionally easier to use, uses the finite dimension

$$
n(\Gamma, q):=\operatorname{dim}_{\mathbb{C}}\left(I^{q+1} \backslash I^{q}\right) .
$$

$V$ is maximally perturbable if and only if $V^{\Gamma, q+1} / V^{\Gamma, q} \cong\left(V^{\Gamma}\right)^{n(\Gamma, q)}$ for all $q \in \mathbb{N}$.

The numbers $n(\Gamma, q)$ are determined by the algebraic structure of the group $\Gamma$, and increase quickly with $q$ for many discrete $\Gamma \subset \mathrm{PSL}_{2}(\mathbb{R})$. For a maximally perturbable $\Gamma$-module with a nonzero space $V^{\Gamma}$ of invariants, the sizes of the spaces of higher-order invariants $V^{\Gamma, q}$ also increase quickly, restricted only by the exact sequence in (3-3).

In [Diamantis and Sim 2008], higher-order cusp forms of weight $k$ for a discrete group $\Gamma$ are considered in the space of holomorphic functions on $\mathfrak{H}$ with exponential decay at the cusps that moreover are invariant under the parabolic 
transformations. The dimensions of these spaces are computed and generally turn out to be strictly smaller than $n(\Gamma, q)$ times the dimension of the spaces of invariants. So the corresponding $\Gamma$-module is not maximally perturbable.

The map $\varphi \mapsto\left(\left(\gamma_{1}, \ldots, \gamma_{1}\right) \mapsto \varphi \mid\left(\gamma_{1}-1\right) \ldots\left(\gamma_{q}-1\right)\right)$ induces an isomorphism $\operatorname{hom}_{\mathbb{C}[\Gamma]}\left(I^{q+1} \backslash I^{q}, V^{\Gamma}\right) \cong \operatorname{Mult}^{q}\left(\Gamma, V^{\Gamma}\right)$, where $\operatorname{Mult}^{q}\left(\Gamma, V^{\Gamma}\right)$ is the space of maps $\Gamma^{q} \rightarrow V^{\Gamma}$ inducing group homomorphisms $\Gamma \rightarrow \mathbb{C}$ on each of their coordinates. For a finitely generated group $\Gamma$, $\operatorname{Mult}^{q}\left(\Gamma, V^{\Gamma}\right) \cong \operatorname{Mult}^{q}(\Gamma, \mathbb{C}) \otimes_{\mathbb{C}} V^{\Gamma}$, where $\operatorname{Mult}^{q}(\Gamma, \mathbb{C})$ is the $q$-th tensor power of $\operatorname{hom}(\Gamma, \mathbb{C})$. This description suggests that it may be useful to consider the following special higher-order invariants:

Definition 3.2. Let $q \in \mathbb{N}$. For any group $\Gamma$ and any $\Gamma$-module $V$, we call $f \in$ $V^{\Gamma, q}$ a perturbation of $\varphi \in V^{\Gamma}$ if there exists $\mu_{f} \in \operatorname{Mult}^{q}(\Gamma, \mathbb{C})$ such that for all $\gamma_{1}, \ldots, \gamma_{q} \in \Gamma$

$$
f \mid\left(\gamma_{1}-1\right) \ldots\left(\gamma_{q}-1\right)=\mu_{f}\left(\gamma_{1}, \ldots, \gamma_{q}\right) \varphi .
$$

We call a perturbation commutative if $\mu_{f}$ is invariant under all permutations of its arguments. If not, we call it noncommutative.

3B. Canonical generators. In this section, we recall the "canonical generators" of cofinite discrete subgroups of $\mathrm{PSL}_{2}(\mathbb{R})$, and use them to show that certain modules are maximally perturbable.

Let $\Gamma \subset \mathrm{PSL}_{2}(\mathbb{R})$ be a cofinite discrete group of motions in the upper half-plane $\mathfrak{H}$. A system of canonical generators for $\Gamma$ consists of:

- Parabolic generators $P_{1}, \ldots, P_{n_{\mathrm{par}}}$, each conjugate in $\mathrm{PSL}_{2}(\mathbb{R})$ to $\pm\left(\begin{array}{ll}1 & 1 \\ 0 & 1\end{array}\right)$. We shall assume that $\Gamma$ has cusps: $n_{\text {par }} \geq 1$.

- Elliptic generators $E_{1}, \ldots, E_{n_{\mathrm{ell}}}$, with $n_{\mathrm{ell}} \geq 0$. Each $E_{j}$ is conjugate to $\pm\left(\begin{array}{c}\cos \left(\pi / v_{j}\right) \sin \left(\pi / v_{j}\right) \\ -\sin \left(\pi / v_{j}\right) \cos \left(\pi / v_{j}\right)\end{array}\right)$ in $\mathrm{PSL}_{2}(\mathbb{R})$ for some $v_{j} \geq 2$.

- Hyperbolic generators $H_{1}, \ldots, H_{2 g}$, with $g \geq 0$, each conjugate in $\operatorname{PSL}_{2}(\mathbb{R})$ to the image $\pm\left(\begin{array}{cc}t & 0 \\ 0 & t^{-1}\end{array}\right), t>1$, of a diagonal matrix.

See, for example, [Lehner 1964, Chapter VII.4, p. 241] or [Petersson 1948, §3]. The relations are given by the condition that each $E_{j}^{v_{j}}$ equals Id for $j=1, \ldots, n_{\mathrm{ell}}$, and one large relation

$$
P_{1} \ldots P_{n_{\mathrm{par}}} E_{1} \ldots E_{n_{\mathrm{ell}}}\left[H_{1}, H_{2}\right] \ldots\left[H_{2 g-1} H_{2 g}\right]=\mathrm{Id} .
$$

The choice of canonical generators is not unique, but the numbers $n_{\mathrm{par}}, n_{\mathrm{ell}}$ and $g$, and the elliptic orders $v_{1}, \ldots, v_{n_{\mathrm{ell}}}$, are uniquely determined by $\Gamma$.

Each group homomorphism $\Gamma \rightarrow \mathbb{C}$ vanishes on the $E_{j}$, and is determined by its values on $H_{1}, \ldots, H_{2 g}, P_{1}, \ldots, P_{n_{\text {par }}-1}$; hence, since $\Gamma$ has cusps,

$$
\operatorname{dim} \operatorname{hom}(\Gamma, \mathbb{C})=n_{\mathrm{par}}-1+2 g .
$$


We put $t(\Gamma)=n_{\text {par }}+2 g$ and denote $P_{1}$ by $A_{1}, \ldots, P_{n_{\mathrm{par}}-1}$ by $A_{n_{\mathrm{par}}-1}, H_{1}$ by $A_{n_{\mathrm{par}}}, \ldots, H_{2 g}$ by $A_{t(\Gamma)-1}$. The group $\Gamma$ is generated by $E_{1}, \ldots, E_{n_{\mathrm{ell}}}$ and $A_{1}, \ldots, A_{t(\Gamma)-1}$.

For the modular group, we have $n_{\mathrm{par}}=1, P_{1}= \pm\left(\begin{array}{ll}1 & 1 \\ 0 & 1\end{array}\right), n_{\mathrm{ell}}=2, E_{1}= \pm\left(\begin{array}{rr}1 & 1 \\ -1 & 0\end{array}\right)$, $E_{2}= \pm S:= \pm\left(\begin{array}{rr}0 & -1 \\ 1 & 0\end{array}\right), g=0$, and hence $\operatorname{hom}\left(\Gamma_{\bmod }, \mathbb{C}\right)=\{0\}$ and $t\left(\Gamma_{\bmod }\right)=1$.

In the sequel, we will need a basis for $I^{q+1} \backslash I^{q}$. Arguing as in Lemma 2.1 in [Deitmar 2009], we can deduce that the elements

$$
\boldsymbol{b}(\boldsymbol{i})=\left(A_{i(1)}-1\right) \ldots\left(A_{\boldsymbol{i}(q)}-1\right),
$$

where $\boldsymbol{i}$ runs over all $(t(\Gamma)-1)$-tuples of elements of $\{1, \ldots, t(\Gamma)-1\}$, form a basis of $I^{q+1} \backslash I^{q}$. We do not give a proof here, since it follows from the more general result in Proposition 5.1.

\section{Maass forms}

We turn to spaces of functions on the upper half-plane that contain the classical holomorphic automorphic forms and the more general Maass forms. The first main results of this paper are stated in Theorems 4.2 and 4.3. In Section 4C, we give some explicit examples of higher-order Maass forms.

4A. General Maass forms. Let $\Gamma$ be a cofinite discrete subgroup $\Gamma$ of the group $G=\mathrm{PSL}_{2}(\mathbb{R})$. For each cusp $\kappa$, we choose $g_{\kappa} \in \mathrm{PSL}_{2}(\mathbb{R})$ such that

$$
\kappa=g_{\kappa} \infty \quad \text { and } \quad g_{\kappa}^{-1} \Gamma_{\kappa} g_{\kappa}=\left\{ \pm\left(\begin{array}{ll}
1 & n \\
0 & 1
\end{array}\right): n \in \mathbb{Z}\right\} .
$$

Here, $\Gamma_{\kappa}$ is the set of elements of $\Gamma$ fixing $\kappa$. The elements $g_{\kappa}$ are determined up to right multiplication by elements $\pm\left(\begin{array}{cc}a & b \\ 0 & a^{-1}\end{array}\right) \in G$. We choose the $g_{\kappa}$ for cusps in the same $\Gamma$-orbit so that $g_{\gamma \kappa} \in \gamma g_{\kappa} \Gamma_{\infty}$.

We further consider a generalization of the action $\mid$ considered in the last section. For a fixed $k$ and for a $f: \mathfrak{H} \rightarrow \mathbb{C}$, we set

$$
\left.f\right|_{k}\left(\begin{array}{ll}
a & b \\
c & d
\end{array}\right)(z)=(c z+d)^{-k} f\left(\frac{a z+b}{c z+d}\right) .
$$

We finally set

$$
L_{k}=-y^{2} \partial_{x}^{2}-y^{2} \partial_{y}^{2}+i k y \partial_{x}-k y \partial_{y}+\frac{k}{2}\left(1-\frac{k}{2}\right) .
$$

With this notation, we have:

Definition 4.1. Let $k \in 2 \mathbb{Z}$ and $\lambda \in \mathbb{C}$.

i) $\mathcal{M}_{k}(\Gamma, \lambda)$ denotes the space of smooth functions $f: \mathfrak{H} \rightarrow \mathbb{C}$ such that $L_{k} f=\lambda f$ and for which there is some $a \in \mathbb{R}$ such that

$$
f\left(g_{\kappa}(x+i y)\right)=\mathrm{O}\left(y^{a}\right), \quad(y \rightarrow \infty)
$$


uniformly for $x$ in compact sets in $\mathbb{R}$, for all cusps $\kappa$ of $\Gamma$.

ii) $\mathscr{E}_{k}(\Gamma, \lambda)$ denotes the space of smooth functions $f$ such that $L_{k} f=\lambda f$ and for which there is some $a \in \mathbb{R}$ such that

$$
f\left(g_{\kappa}(x+i y)\right)=\mathrm{O}\left(e^{a y}\right), \quad(y \rightarrow \infty)
$$

uniformly for $x$ in compact sets in $\mathbb{R}$, for all cusps $\kappa$ of $\Gamma$.

iii) We denote the invariants in these spaces by

$$
E_{k}(\Gamma, \lambda):=\mathscr{E}_{k}(\Gamma, \lambda)^{\Gamma} \quad \text { and } \quad M_{k}(\Gamma, \lambda):=M_{k}(\Gamma, \lambda)^{\Gamma} .
$$

We call the elements of $E_{k}(\Gamma, \lambda)$ (resp. $M_{k}(\Gamma, \lambda)$ ) Maass forms of polynomial (resp. exponential) growth of weight $k$ and eigenvalue $\lambda \in \mathbb{C}$ for $\Gamma$.

Remarks. i) Since $L_{k}$ is elliptic, all its eigenfunctions are automatically realanalytic. (See, for example, [Lang 1975, §5 of Appendix A4], and the references therein.) If $f$ is holomorphic, then it is an eigenfunction of $L_{k}$ with eigenvalue $(k / 2)(1-k / 2)$.

ii) The space $M_{k}(\Gamma, \lambda)$ is known to have finite dimension. The space $E_{k}(\Gamma, \lambda)$ has, for groups $\Gamma$ with cusps, infinite dimension. The subspace of $E_{k}(\Gamma, \lambda)$ corresponding to a fixed value of $a$ in the bound $\mathrm{O}\left(e^{a y}\right)$ has finite dimension. (The first statement is due to Maass. See [Maass 1983, p. 190, Theorem 28] for the case that $\Gamma \subset \mathrm{PSL}_{2}(\mathbb{Z})$. The three statements hold for all $\Gamma$. See, for instance, the discussion in Section 9.5 of [Bruggeman 1994].)

iii) In an alternative definition, suitable for functions not necessarily holomorphic, one replaces the Maass forms $f$ as defined above by $h(z)=y^{k / 2} f(z)$. Then invariance under (4-2) becomes invariance under the action

$$
\left.f\right|_{k}\left(\begin{array}{ll}
a & b \\
c & d
\end{array}\right)(z)=e^{-i k \arg (c z+d)} f\left(\frac{a z+b}{c z+d}\right),
$$

and the eigenproperty in terms of the Laplacian

$$
\left(-y^{2} \partial_{x}^{2}-y^{2} \partial_{y}^{2}+i k y \partial_{x}\right) h=\lambda h .
$$

The formulation of the growth conditions remains unchanged. Now antiholomorphic automorphic forms $a(z)$ of weight $k$ give Maass forms $h(z)=y^{k / 2} a(z)$ of weight $-k$.

Our main result for general Maass forms on $\mathfrak{H}$ is:

Theorem 4.2. Let $\Gamma$ be a cofinite discrete group of motions in $\mathfrak{H}$ with cusps. Then the $\Gamma$-module $\mathscr{E}_{k}(\Gamma, \lambda)$ is maximally perturbable for each $k \in 2 \mathbb{Z}$ and each $\lambda \in \mathbb{C}$. In the course of the proof in Section 8, we will see that even if we start with Maass forms with polynomial growth, the construction of higher-order invariants will lead us to functions that have exponential growth. 
4B. Holomorphic automorphic forms. For even $k$, the space $\mathscr{E}_{k}\left(\Gamma, \lambda_{k}\right)$, with $\lambda_{k}=$ $(k / 2)(1-k / 2)$, contains the subspace $\mathscr{E}_{k}$ hol $\left(\Gamma, \lambda_{k}\right)$, where the condition $L_{k} f=\lambda_{k} f$ is replaced by the stronger condition that $f$ is holomorphic. In the alternative definition, condition (4-8) is replaced by the condition that $z \mapsto y^{-k / 2} f(z)$ is holomorphic. The space $\mathscr{E}_{k}$ hol $\left(\Gamma, \lambda_{k}\right)$ is a $\Gamma$-submodule of $\mathscr{E}_{k}\left(\Gamma, \lambda_{k}\right)$. We also have the $\Gamma$-submodule $\mathcal{M}_{k}^{\mathrm{hol}}\left(\Gamma, \lambda_{k}\right)=\mathcal{M}_{k}\left(\Gamma, \lambda_{k}\right) \cap \mathscr{E}_{k}^{\mathrm{hol}}\left(\Gamma, \lambda_{k}\right)$ of $\mathcal{M}_{k}\left(\Gamma, \lambda_{k}\right)$.

The space $\mu_{k}^{\text {hol }}\left(\Gamma, \lambda_{k}\right)^{\Gamma}$ is the usual space of entire weight- $k$ automorphic forms for $\Gamma$, and $\mathscr{E}_{k} \mathrm{hol}\left(\Gamma, \lambda_{k}\right)^{\Gamma}$ is the space of meromorphic automorphic forms with singularities only at cusps. Sometimes, as in [Bruinier et al. 2008], the elements of $\mathscr{E}_{k}$ hol $\left(\Gamma, \lambda_{k}\right)^{\Gamma}$ are called weakly holomorphic. There the elements of $\mathscr{E}_{k}\left(\Gamma, \lambda_{k}\right)^{\Gamma}$ are called harmonic weak Maass forms. We prefer to use the term harmonic for Maass forms in $\mathscr{E}_{k}(\Gamma, 0)^{\Gamma}$. (Note that $\lambda_{k} \neq 0$ for $k \neq 0,2$.)

Our main result for holomorphic automorphic forms on $\mathfrak{H}$ is:

Theorem 4.3. Let $\Gamma$ be a cofinite discrete group of motions in $\mathfrak{H}$ with cusps. Then $\mathscr{E}_{k}$ hol $\left(\Gamma, k / 2-k^{2} / 4\right)$ is maximally perturbable for each $k \in 2 \mathbb{Z}$.

4C. Examples of harmonic and holomorphic forms of orders 2 and 3. According to Theorems 4.2 and 4.3, there are plenty of examples of higher-order Maass forms for cofinite groups with cusps for which $\operatorname{dim}_{\mathbb{C}} \operatorname{hom}(\Gamma, \mathbb{C}) \geq 1$. (See the discussion following Definition 3.1.) It is, however, not very easy to exhibit explicit examples.

For the modular group $\Gamma_{\text {mod }}=\operatorname{PSL}_{2}(\mathbb{Z})$, the space $\operatorname{hom}\left(\Gamma_{\text {mod }}, \mathbb{C}\right)$ is zero. Hence, it does not accept higher-order invariants. For the commutator subgroup $\Gamma_{\text {com }}=$ $\left[\Gamma_{\text {mod }}, \Gamma_{\text {mod }}\right]$, we will employ three different approaches to exhibit full sets of perturbations of 1 (as defined in Definition 3.2) of orders 2 and 3. A reader only interested in the existence of higher-order forms may prefer to skip this subsection.

4C1. Holomorphic perturbation of 1. In [Lehner 1964, Chapter XI, §3E, p. 362], one finds various facts concerning $\Gamma_{\text {com. }}$. It is freely generated by $D= \pm\left(\begin{array}{ll}2 & 1 \\ 1 & 1\end{array}\right)$ and $C= \pm\left(\begin{array}{rr}2 & -1 \\ -1 & 1\end{array}\right)$. It has no elliptic elements, and one cuspidal orbit $\Gamma_{\text {com }} \infty=\mathbb{P}_{\mathbb{Q}}^{1}$. The group $\left(\Gamma_{\text {com }}\right)_{\infty}$ fixing $\infty$ is generated by $\pm\left(\begin{array}{ll}1 & 6 \\ 0 & 1\end{array}\right)$. We have $t\left(\Gamma_{\text {com }}\right)=3$.

The space of holomorphic cusp forms of weight 2 has dimension $g=1$. We use the basis element $\eta^{4}$ (power of the Dedekind eta function). The map

$$
H(z)=-2 \pi i \int_{\infty}^{z} \eta(\tau)^{4} d \tau=-6 e^{\pi i z / 3}+\mathrm{O}\left(e^{7 \pi i z / 3}\right)
$$

induces an embedding of $\Gamma_{\text {com }} \backslash \mathfrak{H}$ into an elliptic curve, which can be described as $\mathbb{C} / \Lambda$, with

$$
\Lambda=\varpi \mathbb{Z}[\rho], \quad \varpi=\pi^{1 / 2} \Gamma\left(\frac{1}{6}\right) /\left(6 \sqrt{3} \Gamma\left(\frac{2}{3}\right)\right), \quad \rho=e^{\pi i / 3} .
$$


(See computations in [Bruggeman 1994, §15.2-3].) The map $H$ maps $\mathfrak{H}$ onto $\mathbb{C} \backslash \Lambda$, and satisfies for $\gamma \in \Gamma_{\text {com }}$

$$
H(\gamma z)=H(z)+\lambda(\gamma), \quad \lambda(\gamma)=-2 \pi i \int_{\infty}^{\gamma \infty} \eta(\tau)^{4} d \tau,
$$

where $\lambda(C)=\rho \varpi$ and $\lambda(D)=\bar{\rho} \varpi$. So the lattice $\Lambda$ is the image of $\lambda: \Gamma_{\text {com }} \rightarrow \mathbb{C}$, and $\operatorname{hom}\left(\Gamma_{\text {com }}, \mathbb{C}\right)=\operatorname{Mult}^{1}\left(\Gamma_{\text {com }}, \mathbb{C}\right)$ has $\lambda, \bar{\lambda}$ as a basis. We note that the kernel $\operatorname{ker}(\lambda)$ is a subgroup with infinite index in $\Gamma_{\text {com }}$; it is in fact the commutator subgroup of $\Gamma_{\text {com }}$. The element $\pm\left(\begin{array}{ll}1 & 6 \\ 0 & 1\end{array}\right)$ generating the subgroup of $\Gamma_{\text {com }}$ fixing $\infty$ is in $\operatorname{ker}(\lambda)$. Since $\operatorname{ker}(\lambda)$ has no elliptic elements, composition with $H$ gives a bijection from the holomorphic functions on $\mathbb{C} \backslash \Lambda$ to the holomorphic $\operatorname{ker}(\lambda)$-invariant functions on $\mathfrak{H}$.

Clearly, $H$ is a holomorphic second-order perturbation of 1 with linear form $\lambda$. It is also a harmonic perturbation of 1 , that is, a perturbation which is harmonic as a function. By conjugation, we obtain the antiholomorphic harmonic perturbation of 1 with linear form $\bar{\lambda}$.

According to Theorem 4.3, there should also be a holomorphic second-order perturbation of 1 with a linear form that is linearly independent of $\lambda$. Here we can use the Weierstrass zeta function

$$
\zeta(u ; \Lambda)=\frac{1}{u}+\sum_{\omega \in \Lambda}^{\prime}\left(\frac{1}{u-\omega}+\frac{1}{\omega}+\frac{u}{\omega^{2}}\right) .
$$

See, for example, [Koecher and Krieg 1998, Chapter I, §6]. It is holomorphic on $\mathbb{C} \backslash \Lambda$ and satisfies $\zeta(u+\omega ; \Lambda)=\zeta(u ; \Lambda)+\mathrm{h}(\omega)$ for all $\omega \in \Lambda$, where $\mathrm{h} \in \operatorname{hom}(\Lambda, \mathbb{C})$ is linearly independent of $\omega \mapsto \omega$. (The classical notation for $h$ is $\eta$. We write $h$ to avoid confusion with the Dedekind eta function.) Pulling back this zeta function to $\mathfrak{H}$, we get a second-order holomorphic perturbation of 1 :

$$
W(z)=\zeta(H(z) ; \Lambda),
$$

with the linear form $\gamma \mapsto h(\lambda(\gamma))$. The Laurent expansion of the Weierstrass zeta function at 0 starts with $\zeta(u ; \Lambda)=u^{-1}+\mathrm{O}\left(u^{3}\right)$. Hence, $W$ has a Fourier expansion at $\infty$ starting with

$$
W(z)=-\frac{1}{6} e^{-\pi i z / 3}+\mathrm{O}\left(e^{\pi i z}\right) .
$$

This shows that $W$ has exponential growth at the cusps.

We may carry this out also for holomorphic forms of order 3 , to obtain the following commutative perturbations of 1 of order 3 :

\begin{tabular}{|c|c|c|c|}
\hline$f$ & $H(z)^{2}$ & $H(z) W(z)$ & $W(z)^{2}$ \\
$\mu_{f}$ & $2 \lambda \otimes \lambda$ & $\lambda \otimes(\mathrm{h} \circ \lambda)+(\mathrm{h} \circ \lambda) \otimes \lambda$ & $2(\mathrm{~h} \circ \lambda) \otimes(\mathrm{h} \circ \lambda)$ \\
\hline
\end{tabular}


We know that there also exist noncommutative holomorphic perturbations of order 3. To find an explicit example, we have to work on $\mathfrak{H}$, since the group $\Lambda$ acting on $\mathbb{C}$ is abelian.

The closed holomorphic 1-forms

$$
\omega=-2 \pi i \eta(\tau)^{4} d \tau \quad \text { and } \quad \omega_{1}=-2 \pi i W(\tau) \eta(\tau)^{4} d \tau
$$

on $\mathfrak{H}$ transform as follows under $\Gamma_{\text {com }}$ :

$$
\omega\left|\gamma=\omega, \quad \omega_{1}\right| \gamma=\omega_{1}+\mathrm{h}(\lambda(\gamma)) \omega .
$$

For an arbitrary base point $z_{0} \in \mathfrak{H}$, we put

$$
K(z)=\int_{z_{0}}^{z} \omega_{1}
$$

This defines a holomorphic function on $\mathfrak{H}$ that satisfies, for $\gamma \in \Gamma_{\text {com }}$,

$$
K \mid(\gamma-1)(z)=\int_{z}^{\gamma z} \omega_{1}
$$

and hence for $\gamma, \delta \in \Gamma_{\mathrm{com}}$,

$$
\begin{aligned}
K \mid(\gamma-1)(\delta-1)(z) & =\left(\int_{\gamma z}^{\gamma \delta z}-\int_{z}^{\delta z}\right) \omega_{1}=\int_{z}^{\delta z} \omega_{1} \mid \gamma-\int_{z}^{\delta z} \omega_{1} \\
& =\mathrm{h}(\lambda(\gamma)) \int_{z}^{\delta z} \omega=\mathrm{h}(\lambda(\gamma)) \lambda(\delta) .
\end{aligned}
$$

Thus, we have a holomorphic third-order noncommutative perturbation $K$ of 1 with nonsymmetric multilinear form $(h \circ \lambda) \otimes \lambda$. Since holomorphic forms are harmonic in weight zero, these perturbations are also harmonic perturbations of 1 .

4C2. Iterated integrals. The construction of the third-order form $K$ in (4-17) is closely related to the iterated integrals used in [Diamantis and Sreekantan 2006] to prove maximal perturbability of spaces of smooth functions.

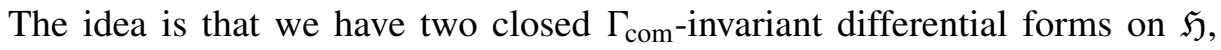
$d H(z)=\omega=-2 \pi i \eta(z)^{4} d z$ and

$$
\omega_{0}=d W(z)=-\wp(H(z)) d(H(z)),
$$


where $\wp(u ; \Lambda)=-(d / d u) \zeta(u ; \Lambda)$ is the Weierstrass $\wp$-function. If $t \mapsto z(t)$, $0 \leq t \leq 1$ is a path in $\mathfrak{H}$ from $z_{0}$ to $z_{1}$, then

$$
\begin{aligned}
\int_{t_{2}=0}^{1} \int_{t_{1}=0}^{t_{2}} \omega_{0}\left(z\left(t_{1}\right)\right) \omega\left(z\left(t_{2}\right)\right) & =\int_{t_{2}=0}^{1}\left(W\left(z\left(t_{2}\right)\right)-W\left(z_{0}\right)\right) d H\left(z\left(t_{2}\right)\right) \\
& =-2 \pi i \int_{t=0}^{1} W(z(t)) \eta(z(t))^{4} z^{\prime}(t) d t \\
& =K\left(z_{1}\right)-W\left(z_{0}\right)\left(H\left(z_{1}\right)-H\left(z_{0}\right)\right)
\end{aligned}
$$

depends only on $z_{0}$ and $z_{1}$, not on the actual path. For a fixed base point $z_{0}$, the holomorphic function $z_{1} \mapsto W\left(z_{0}\right)\left(H\left(z_{1}\right)-H\left(z_{0}\right)\right)$ is invariant of order 2. So up to lower-order terms, the invariant $K$ is given by an iterated integral, as in (3) of [Diamantis and Sreekantan 2006]; see also [Chen 1971].

4C3. Differentiation of families. We start by considering a general finitely generated group $\Gamma$ acting on a space $X$. We will use the notation $f \mid \gamma(x)=f(\gamma x)$ for the action induced on functions defined on $X$. We consider a family of characters of $\Gamma$ of the form $\chi_{r}(\gamma)=e^{i r \cdot \alpha(\gamma)}$, where $r \cdot \alpha(\gamma)=r_{1} \alpha_{1}(\gamma)+\cdots+r_{n} \alpha_{n}(\gamma)$ for $\alpha_{1}, \ldots, \alpha_{n} \in \operatorname{hom}(\Gamma, \mathbb{R})$ and $r$ varying over an open set $U$ in $\mathbb{R}^{n}$. In this way, $\chi_{r}$ is a family of unitary characters.

We consider a $C^{\infty}$ family $r \mapsto f_{r}$ on a neighborhood $U \subset \mathbb{R}^{n}$ of 0 of functions $X \rightarrow \mathbb{C}$ that satisfy

$$
f_{r}(\gamma x)=\chi_{r}(\gamma) f_{r}(x), \quad(\gamma \in \Gamma) .
$$

We assume that $\chi_{0}$ is the trivial character and that $f_{0}$ is a $\Gamma$-invariant function $f$.

We now set $h(x)=\left.\partial_{r_{j}} f_{r}(x)\right|_{r=0}$, for one of the coordinates of $r$. The transformation behavior gives $h(\gamma x)=i \alpha_{j}(\gamma) f(x)+h(x)$, or, rewritten,

$$
h \mid \gamma-h=i \alpha_{j}(\gamma) f \text {. }
$$

The function $h$ is a second-order perturbation of $f$, with $i \alpha_{j}$ as the corresponding element of hom $(\Gamma, \mathbb{C})$. This can be generalized using a routine inductive argument:

Proposition 4.4. For all multi-indices $a \in \mathbb{N}^{n}$, the derivative

$$
f^{(a)}(x):=\left.\partial_{r}^{a} f_{r}(x)\right|_{r=0}
$$

is a commutative perturbation of $f$ with order $1+|a|$. Here $\partial_{r}^{a}=\partial_{r_{1}}^{a_{1}} \ldots \partial_{r_{n}}^{a_{n}}$ and $|a|=a_{1}+a_{2}+\cdots+a_{n}$.

Remark. Proposition 4.4 shows that commutative perturbations can arise as infinitesimal perturbations of a family of automorphic forms. That is our motivation to use the word perturbation in Definition 3.2. 
Application to harmonic perturbations of 1. We use the method of differentiation of families to produce explicit harmonic higher-order forms for $\Gamma_{\text {com }}$ of order 3 . We employ families studied in [Bruggeman 1994].

Since $\Gamma_{\text {com }}$ is free on the generators $C= \pm\left(\begin{array}{rr}2 & -1 \\ -1 & 1\end{array}\right)$ and $D= \pm\left(\begin{array}{ll}2 & 1 \\ 1 & 1\end{array}\right)$, the character group of $\Gamma_{\text {com }}$ is isomorphic to $\mathbb{C}^{*} \times \mathbb{C}^{*}$. We can parametrize the characters by

$$
\chi_{v, w}(\gamma)=e^{i v \lambda(\gamma)+i w \overline{\lambda(\gamma)}}
$$

where $(v, w)$ runs through $\mathbb{C}^{2}$, and where $\lambda \in \operatorname{hom}\left(\Gamma_{\text {com }}, \mathbb{C}\right)$ is as defined in (4-11). We are interested only in $(v, w)$ in a neighborhood of $0 \in \mathbb{C}^{2}$.

In [Bruggeman 1994, §15.5], it is shown that there is a meromorphic Eisenstein family $E(v, w, s)$ of automorphic forms for $\Gamma_{\text {com }}$, with the character $\chi_{v, w}$ and eigenvalue $\frac{1}{4}-s^{2}$ for $\omega_{0}=-y^{2}\left(\partial_{x}^{2}+\partial_{y}^{2}\right)$. (In [Bruggeman 1994], the discussion of the family $E$ is made in the context of families of automorphic forms of varying weight which are thus defined on the covering group $\tilde{\Gamma}_{\text {com }}$. However, in $\S 15.5$ the weight is zero, and the automorphic forms are, in effect, on the discrete group $\Gamma_{\text {com. }}$.) The restriction to $s=\frac{1}{2}$ exists [Bruggeman 1994, §15.6] and forms a meromorphic family $(v, w) \mapsto f(v, w ; z)$ on $\mathbb{C}^{2}$ such that $f(v, w ; \gamma z)=\chi_{v, w}(\gamma) f(v, w ; z)$, and $L_{0} f(v, w ; z)=0$ for the dense set of $(v, w)$ at which $f$ is holomorphic. There is a meromorphic family $(v, w) \mapsto h(v, w ; \cdot)$ on $\mathbb{C}$ such that

$$
f(v, w ; z)=h(v, w ; H(z)),
$$

satisfying $h(v, w ; u+\lambda)=e^{i v \lambda+i w \bar{\lambda}} h(v, w ; u)$ [Bruggeman 1994, §15.1-6]. Chapter 15 of [Bruggeman 1994] gives a complicated but explicit construction (obtained with the help of D. Zagier) of such a family $h$ with Jacobi theta functions.

Specifically, in $\S 15.6 .11$, the function $h$ is expressed as a sum

$$
h(v, w ; u)=G_{(v+w) \varpi / 2 \pi}(u, w)+G_{-(v+w) \varpi / 2 \pi}(-\bar{u},-v),
$$

where the function $G_{\mu}(u, w)$, for $\mu \notin \mathbb{Z}$ and $0<\operatorname{Im} u<\frac{1}{2} \varpi \sqrt{3}$, is given by

$$
G_{\mu}(u, w)=\sum_{m=-\infty}^{\infty} \frac{1}{\mu+m} \frac{\xi^{\mu+m}}{\eta q^{m}-1},
$$

with $q=-e^{-\pi \sqrt{3}}, \xi=e^{2 \pi i u / \varpi}$, and $\eta=e^{-w \varpi \sqrt{3}}$. We compute a part of the expansion in powers of $v$ and $w$ at $v=w=0$. With the substitution $u=H(z)$, the coefficients provide us with higher-order harmonic modular forms for $\Gamma_{\text {com. }}$. Some 
of these we have seen above. Denoting $f=-2 \pi / \varpi^{2} \sqrt{3}$, we find:

\begin{tabular}{|c|c|l|}
\hline term of & on $\mathbb{C}$ & \multicolumn{1}{|c|}{ on $\mathfrak{H}$} \\
\hline 1 & $f$ & $f$ (constant function) \\
$v$ & if $u$ & if $H(z)$ \\
$w$ & if $\bar{u}$ & if $\overline{H(z)}$ \\
$v^{2}$ & $(-f / 2) u^{2}$ & $(-f / 2) H(z)^{2}$ \\
$w^{2}$ & $(-f / 2) \bar{u}^{2}$ & $(-f / 2) \overline{H(z)}^{2}$ \\
\hline
\end{tabular}

The coefficient of $v w$ gives a third-order form

$$
\begin{aligned}
b_{1,1}(u):= & \frac{\pi}{\sqrt{3}}\left(\left(\frac{u}{\varpi}-\frac{i \sqrt{3}}{2}\right)^{2}+\left(\frac{\bar{u}}{\varpi}+\frac{i \sqrt{3}}{2}\right)^{2}+1\right) \\
& +S(u)+S(\varpi \rho-u)+S(-\bar{u})+S(\varpi \rho+\bar{u}),
\end{aligned}
$$

with

$$
S(u):=\sum_{m=1}^{\infty} \frac{e^{2 \pi i m u / \varpi}}{m\left(q^{m}-1\right)}, \quad \rho=\frac{1}{2}+\frac{i}{2} \sqrt{3} .
$$

By $B_{1,1}(z)=b_{1,1}(H(z))$ we denote the corresponding harmonic third-order perturbation of 1 on $\mathfrak{H}$. The way $B_{1,1}$ has been derived, together with the proof of Proposition 4.4, ensures that it is a perturbation of 1 with a multilinear form that is a multiple of $\lambda \otimes \bar{\lambda}+\bar{\lambda} \otimes \lambda$.

However, $b_{1,1}(u)$ is represented by (4-23) only on the region $0<\operatorname{Im} u<\frac{1}{2} \varpi \sqrt{3}$. By further computations, we arrive at expressions for it on larger regions, and can determine the associated bilinear form. We then see that the pull-back $-f^{-1} B_{1,1}=$ $-f^{-1} b_{1,1} \circ H$ is a harmonic commutative perturbation of 1 for the multilinear form $\mu$ determined by the following values at the generators $C$ and $C D$ of $\Gamma_{\text {com: }}$ :

$$
\mu(g, h)= \begin{cases}2 \varpi^{2} & \text { if } g=h=C \text { or } C D, \\ \varpi^{2} & \text { if } g=C, h=C D, \text { or if } g=C D, h=C .\end{cases}
$$

We have used the values of $\lambda$ given below (4-11). With these values at the generators, $\mu$ coincides with $\lambda \otimes \bar{\lambda}+\bar{\lambda} \otimes \lambda$ as predicted above by the way $B_{1,1}$ was constructed.

Proposition 4.4 shows that differentiation of families produces only commutative perturbations. However, by Theorem 4.2, there are noncommutative third-order harmonic perturbations of 1 . We can obtain such perturbations from $B_{1,1}$ upon decomposing it as $B_{1,1}=A+B$ for a holomorphic function $A$ and an antiholomorphic function $B$. Specifically, in view of (4-23), for those $z \in \mathfrak{H}$ for which $H(z)$ is 
in the upper half of the fundamental hexagon for $\mathbb{C} / \Lambda$, we can set

$$
\begin{aligned}
& A(z)=\frac{\pi}{2 \sqrt{3}}+\frac{\pi}{\sqrt{3}}\left(\frac{H(z)}{\varpi}-\frac{i \sqrt{3}}{2}\right)^{2}+S(H(z))+S(\varpi \rho-H(z)), \\
& B(z)=\frac{\pi}{2 \sqrt{3}}+\frac{\pi}{\sqrt{3}}\left(\frac{\overline{H(z)}}{\varpi}+\frac{i \sqrt{3}}{2}\right)^{2}+S(-\overline{H(z)})+S(\varpi \rho+\overline{H(z)}) .
\end{aligned}
$$

More computations lead to the conclusion that $-f^{-1} A$ is a noncommutative holomorphic third-order holomorphic perturbation of 1 with multilinear form $\bar{\lambda} \otimes \lambda$, and that the multilinear form of the anticommutative third-order perturbation of 1 given by $-f^{-1} B=-f^{-1}\left(B_{1,1}-A\right)$ is $(\lambda \otimes \bar{\lambda}+\bar{\lambda} \otimes \lambda)-\bar{\lambda} \otimes \lambda=\lambda \otimes \bar{\lambda}$.

\section{Universal covering group}

5A. Universal covering group of $\mathrm{SL}_{2}(\mathbb{R})$. For our purpose, it suffices to describe the universal covering group $\tilde{G}$ of $\mathrm{SL}_{2}(\mathbb{R})$ as the Lie group with underlying analytic space the product $\mathfrak{H} \times \mathbb{R}$ with the group operations uniquely defined by the requirements that $(i, 0)$ be the unit element and that

$$
\operatorname{pr}_{2}(z, \vartheta)=\left(\begin{array}{cc}
\sqrt{y} & x / \sqrt{y} \\
0 & 1 / \sqrt{y}
\end{array}\right)\left(\begin{array}{rr}
\cos \vartheta & \sin \vartheta \\
-\sin \vartheta & \cos \vartheta
\end{array}\right)
$$

be a surjective group homomorphism $\operatorname{pr}_{2}: \tilde{G} \rightarrow \mathrm{SL}_{2}(\mathbb{R})$. We will often use the lift $g \mapsto \tilde{g}: \mathrm{SL}_{2}(\mathbb{R}) \rightarrow \tilde{G}$ given by

$$
\widetilde{\left(\begin{array}{ll}
a & b \\
c & d
\end{array}\right)}=\left(\frac{a i+b}{c i+d},-\arg (c i+d)\right),
$$

with the convention that the argument takes values in $(-\pi, \pi]$. It satisfies, for all $(z, \vartheta) \in \tilde{G}$,

$$
\widetilde{\left(\begin{array}{ll}
a & b \\
c & d
\end{array}\right)}(z, \vartheta)=\left(\frac{a z+b}{c z+d}, \vartheta-\arg (c z+d)\right) .
$$

By pr: $\tilde{G} \rightarrow \mathrm{PSL}_{2}(\mathbb{R})$ we denote the composition of $\mathrm{pr}_{2}$ and the natural map $\mathrm{SL}_{2}(\mathbb{R}) \rightarrow \mathrm{PSL}_{2}(\mathbb{R})$.

We will use the following homomorphisms of Lie groups:

$$
\begin{aligned}
n: \mathbb{R} \rightarrow \tilde{G}, & n(x)=(x+i, 0), & \operatorname{pr} n(x) & =\left[\begin{array}{ll}
1 & x \\
0 & 1
\end{array}\right], \\
a: \mathbb{R}_{>0}^{*} \rightarrow \tilde{G}, & a(y)=(i y, 0), & \operatorname{pr} a(y) & =\left[\begin{array}{cc}
\sqrt{y} & 0 \\
0 & 1 / \sqrt{y}
\end{array}\right], \\
k: \mathbb{R} \rightarrow \tilde{G}, & k(\vartheta)=(0, \vartheta), & \operatorname{pr} k(\vartheta) & =\left[\begin{array}{cc}
\cos \vartheta & \sin \vartheta \\
-\sin \vartheta & \cos \vartheta
\end{array}\right] .
\end{aligned}
$$


The Lie algebra of $\tilde{G}$ is isomorphic to the Lie algebra of $\mathrm{SL}_{2}(\mathbb{R})$ and of $\mathrm{PSL}_{2}(\mathbb{R})$. A basis of the complex Lie algebra is $\boldsymbol{W}, \boldsymbol{E}^{+}, \boldsymbol{E}^{-}$, with $\boldsymbol{W}$ corresponding to $\left(\begin{array}{rr}0 & 1 \\ -1 & 0\end{array}\right)$ in the Lie algebra of $\mathrm{SL}_{2}(\mathbb{R})$, and $\boldsymbol{E}^{ \pm}$corresponding to $\left(\begin{array}{rr}1 & 0 \\ 0 & -1\end{array}\right) \pm i\left(\begin{array}{ll}0 & 1 \\ 1 & 0\end{array}\right)$. The corresponding left-invariant differential operators are, in the coordinates $(x+i y, \vartheta)$ on $\tilde{G}$ :

$$
\boldsymbol{W}=\partial_{\vartheta}, \quad \boldsymbol{E}^{ \pm}=e^{ \pm 2 i \vartheta}\left( \pm 2 i y \partial_{x}+2 y \partial_{y} \mp i \partial_{\vartheta}\right)
$$

The Casimir operator

$$
\omega=-\frac{1}{4} \boldsymbol{E}^{ \pm} \boldsymbol{E}^{\mp}+\frac{1}{4} \boldsymbol{W}^{2} \mp \frac{i}{2} \boldsymbol{W}=-y^{2} \partial_{y}^{2}-y^{2} \partial_{x}^{2}+y \partial_{x} \partial_{\vartheta}
$$

generates the center of the enveloping algebra of the Lie algebra, and determines a differential operator that commutes with left and with right translation.

5B. Cofinite discrete subgroups. To a cofinite discrete subgroup $\Gamma$ of $\mathrm{PSL}_{2}(\mathbb{R})$ we associate its inverse image $\tilde{\Gamma}:=\operatorname{pr}^{-1} \Gamma$ in $\tilde{G}$. This gives a bijective correspondence between cofinite discrete subgroups of $\mathrm{PSL}_{2}(\mathbb{R})$ and cofinite discrete subgroups of $\tilde{G}$ that contain the center $\tilde{Z}=\langle\zeta\rangle$, where $\zeta:=k(\pi)$. The projection pr induces an isomorphism $\Gamma \cong \tilde{\Gamma} / \tilde{Z}$.

As an example, we consider the modular group $\Gamma_{\bmod }=\mathrm{PSL}_{2}(\mathbb{Z})$, with corresponding group $\tilde{\Gamma}_{\text {mod }} \subset \tilde{G}$. It is known that $\operatorname{PSL}_{2}(\mathbb{Z})$ is presented by the generators $S= \pm\left(\begin{array}{rr}0 & -1 \\ 1 & 0\end{array}\right)$ and $T= \pm\left(\begin{array}{ll}1 & 1 \\ 0 & 1\end{array}\right)$ and relations $S^{2}=(T S)^{2}=I$.

Set

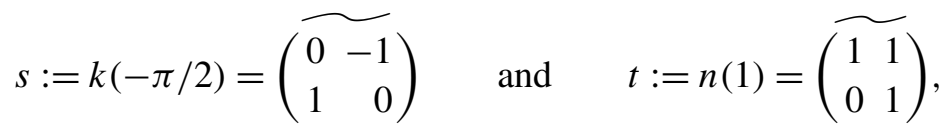

with $\operatorname{pr}(s)=S$ and $\operatorname{pr}(t)=T$. Then $s^{2}=k(-\pi)=\zeta^{-1} \in \tilde{Z}$, so $s$ and $t$ generate $\tilde{\Gamma}_{\text {mod }}$. To determine the relations $s^{2} t=t s^{2}$ and $t s t s t=s$, one carries out a computation using (5-2). This implies that the linear space hom $\left(\tilde{\Gamma}_{\text {mod }}, \mathbb{C}\right)$ has dimension 1 , and is generated by $\alpha: t \mapsto \pi / 6, \alpha: s \mapsto-\pi / 2$. For reasons that will become clear later, we take this basis element, and not an integral-valued one.

5C. Canonical generators. The canonical generators of $\Gamma$ induce canonical generators of $\tilde{\Gamma}$ :

- Elements $\pi_{1}, \ldots, \pi_{n_{\mathrm{par}}}$ of the form $\pi_{j}=\tilde{g}_{\kappa_{j}} n(1) \tilde{g}_{\kappa_{j}}^{-1}$ fixing a system of representatives $\kappa_{1}, \ldots, \kappa_{n_{\text {par }}}$ of the $\tilde{\Gamma}$-orbits of cusps.

- Elements $\varepsilon_{1}, \ldots, \varepsilon_{n_{\text {ell }}}$ conjugate in $\tilde{G}$ to $k\left(\pi / v_{j}\right)$ with $v_{j} \geq 2$.

- Elements $\eta_{1}, \ldots, \eta_{2 g}$ conjugate in $\tilde{G}$ to elements $a\left(t_{j}\right)$ with $t_{j}>1$.

- The generator $\zeta=k(\pi)$ of the center $\tilde{Z}$ of $\tilde{\Gamma}$. 
The relations are:

$\zeta$ is central,

$\varepsilon_{j}^{v_{j}}=\zeta$ for $1 \leq j \leq n_{\mathrm{ell}}$,

$\pi_{1} \ldots \pi_{n_{\mathrm{par}}} \varepsilon_{1} \ldots \varepsilon_{n_{\mathrm{ell}}}\left[\eta_{1}, \eta_{2}\right] \ldots\left[\eta_{2 g-1}, \eta_{2 g}\right]=\zeta^{2 g-2+n_{\mathrm{par}}+n_{\mathrm{ell}}}$.

The integer $2 g-2+n_{\text {par }}+n_{\text {ell }}$ is always positive. For these facts, see [Bruggeman $1994, \S 3.3]$

If $n_{\text {ell }}>0$ or if $2 g-2+n_{\mathrm{par}}=1$ and $n_{\mathrm{ell}}=0$, we do not need $\zeta$ as a generator. If $n_{\text {ell }}=0$, the group $\tilde{\Gamma}$ is free on $\pi_{1}, \ldots, \pi_{n_{\text {par }}-1}, \eta_{1}, \ldots, \eta_{2 g}, \zeta$.

Among the canonical generators we single out the following elements: $\alpha_{1}=\pi_{1}$, $\ldots, \alpha_{n_{\mathrm{par}}-1}=\pi_{n_{\mathrm{par}}-1}, \alpha_{n_{\mathrm{par}}}=\eta_{1}, \ldots, \alpha_{t(\Gamma)-1}=\eta_{2 g}, \alpha_{t(\Gamma)}=\zeta$. (We recall that $t(\Gamma)=n_{\text {par }}+2 g$.) The $\alpha_{j}$ together with the $\varepsilon_{j}$ generate $\tilde{\Gamma}$, with $\varepsilon_{j}^{v_{j}}=\zeta$ and the centrality of $\zeta$ as the sole relations.

For the modular group $\tilde{\Gamma}_{\text {mod }}$, we have $n_{\text {par }}=1, n_{\text {ell }}=2, g=0$, and $t\left(\Gamma_{\text {mod }}\right)=1$. We may take $\pi_{1}=t=n(1), \varepsilon_{1}=t^{-1} s^{-1}$, and $\varepsilon_{2}=s^{-1}=k(\pi / 2)=p^{-1} k(\pi / 3) p$, with $p=n\left(-\frac{1}{2}\right) a\left(\frac{\sqrt{3}}{2}\right)$.

By $I$ we now denote the augmentation ideal of the group ring $\mathbb{C}[\tilde{\Gamma}]$. In $\mathbb{C}[\tilde{\Gamma}]$ we have the elements

$$
\boldsymbol{b}(\boldsymbol{i})=\left(\alpha_{i(1)}-1\right) \ldots\left(\alpha_{i(q)}-1\right), \quad \boldsymbol{i} \in\{1, \ldots, t(\Gamma)\}^{q} .
$$

We allow ourselves to use the same notation as in (3-8), since from now on we will use $\tilde{\Gamma}$. The centrality of $\zeta$ allows us to move $(\zeta-1)$ through the product. So it suffices to consider only $q$-tuples $\boldsymbol{i}$ for which all $\boldsymbol{i}(l)=t(\Gamma)$ occur at the end. Such $q$-tuples we will call $\tilde{\Gamma}$-q-tuples.

Proposition 5.1. $A \mathbb{C}$-basis of $I^{q+1} \backslash I^{q}$ is induced by the elements

$$
\boldsymbol{b}(\boldsymbol{i})=\left(\alpha_{i(1)}-1\right) \ldots\left(\alpha_{i(q)}-1\right),
$$

where $\boldsymbol{i}$ runs over the $\tilde{\Gamma}$-q-tuples.

Proof. The ideal $I^{q}$ is generated by the products of the form $\left(\gamma_{1}-1\right) \ldots\left(\gamma_{q}-1\right)$, with $\gamma_{1}, \ldots, \gamma_{q} \in \tilde{\Gamma}$ [Deitmar 2009, Lemma 1.1]. With the relation

$$
(\gamma \delta-1)=(\gamma-1)(\delta-1)+(\gamma-1)+(\delta-1),
$$

we can take the $\gamma_{j}$ in a system of generators, for instance $\alpha_{1}, \ldots, \alpha_{t(\Gamma)}, \varepsilon_{1}, \ldots, \varepsilon_{n_{\mathrm{ell}}}$. For the elliptic elements $\varepsilon_{j}$, we use $\zeta-1=\sum_{k=0}^{v_{j}-1} \varepsilon_{j}^{k}\left(\varepsilon_{j}-1\right) \equiv v_{j}\left(\varepsilon_{j}-1\right) \bmod I^{2}$ to see that the $\alpha_{j}$ suffice. (Note that $v_{j}$ is invertible in $\mathbb{C}$.) Since $\alpha_{t(\Gamma)}=\zeta$ is central, we can move all occurrences of $\zeta-1$ to the right to see that the $\boldsymbol{b}(\boldsymbol{i})$ in the proposition generate $I^{q+1} \backslash I^{q}$. 
To see that the $\boldsymbol{b}(\boldsymbol{i})$ are linearly independent over $\mathbb{C}$, we proceed in rewriting terms $\xi\left(\alpha_{i(1)}-1\right) \ldots\left(\alpha_{i(q)}-1\right)$ by replacing $\xi \in R:=\mathbb{C}[\tilde{\Gamma}]$ by $n+\eta$, with $n \in \mathbb{C}$ and $\eta \in I$. In this way, we express each element of $I^{q}$ as a $\mathbb{C}$-linear combination of products of $q$ factors $\alpha_{j}-1$ plus a term in $I^{N}$, with $N>q$. To eliminate $I^{N}$, we consider the $I$-adic completion $\hat{R}$ of $\mathbb{C}[\tilde{\Gamma}]$, with closure $\hat{I}^{q}$ of $I^{q}$. Each element of $\hat{I} \supset I$ is a countable sum of products of a complex number and finitely many factors $\alpha_{j}-1$. Since $\hat{I}^{q+1} \backslash \hat{I}^{q}$ and $I^{q+1} \backslash I^{q}$ are isomorphic, it suffices to prove that the $\boldsymbol{b}(\boldsymbol{i})$ are linearly independent as elements of $\hat{I}^{q+1} \backslash \hat{I}^{q}$.

We suppose that there are $x_{i} \in \mathbb{C}$ for all $q$-tuples $i$ such that

$$
\sum_{i} x_{i}\left(\alpha_{i(1)}-1\right) \ldots\left(\alpha_{i(q)}-1\right) \in \hat{I}^{q+1}
$$

We can write this element of $\hat{I}^{q+1}$ as $\sum_{j} c_{j} \xi_{j}$, with $c_{j} \in \mathbb{C}$ and $\xi_{j}$ running over the countably many products $\left(\alpha_{\boldsymbol{j}(1)}-1\right) \ldots\left(\alpha_{\boldsymbol{j}(m)}-1\right)$ with $m$-tuples from $\{1, \ldots, t(\Gamma)\}$ for all $m>q$.

We form the ring $N=\mathbb{C}\left\langle\Xi_{1}, \ldots, \Xi_{t(\Gamma)}\right\rangle$ of power series in the noncommuting, algebraically independent (over $\mathbb{C}$ ) variables $\Xi_{1}, \ldots, \Xi_{t}$, and the two-sided ideal $Z$ in $N$ generated by the commutators

$$
\Xi_{j} \Xi_{t(\Gamma)}-\Xi_{t(\Gamma)} \Xi_{j}, \quad \text { for } 1 \leq j \leq t(\Gamma) .
$$

The quotient ring $M:=N / Z$ is noncommutative if $t(\Gamma) \geq 3$. The relations between the generators imply that there is a group homomorphism $\varphi: \tilde{\Gamma} \rightarrow M^{*}$ given by $\varphi\left(\alpha_{j}\right)=1+\Xi_{j}$ for $1 \leq j \leq t(\Gamma)$, and

$$
\varphi\left(\varepsilon_{j}\right)=\left(1+\Xi_{t(\Gamma)}\right)^{1 / v_{j}}=\sum_{l \geq 0}\left(\begin{array}{c}
1 / v_{j} \\
l
\end{array}\right) \Xi_{t(\Gamma)}^{l} .
$$

This group homomorphism induces a ring homomorphism $\hat{\varphi}: \hat{R} \rightarrow M$, for which

$$
\hat{\varphi}\left(\xi_{i}\right)=\hat{\varphi}\left(\alpha_{i(1)}-1\right) \hat{\varphi}\left(\alpha_{i(2)}-1\right) \ldots \hat{\varphi}\left(\alpha_{i(|i|)}-1\right)=\Xi^{i}:=\Xi_{i(1)} \Xi_{i(2)} \ldots \Xi_{i(|i|)} .
$$

Now we have

$$
\sum_{i} x_{i} \Xi^{i}=\hat{\varphi}\left(\sum_{i} x_{i} \xi_{i}\right)=\hat{\varphi}\left(\sum_{j} c_{j} \xi_{j}\right)=\sum_{j} c_{j} \Xi^{j}
$$

where $\boldsymbol{i}$ runs over $q$-tuples and $\boldsymbol{j}$ runs over countably many tuples with length strictly larger than $q$. Hence, all $x_{i}$ (and $c_{j}$ ) vanish. 
So for $\tilde{\Gamma}$ with cusps, the trivial $\tilde{\Gamma}$-module $I^{q+1} \backslash I^{q}$ is always nontrivial. The dimension is equal to the number of all $\tilde{\Gamma}-q$-tuples. Thus we have

$$
\begin{aligned}
\operatorname{dim}_{\mathbb{C}}\left(I^{q+1} \backslash I^{q}\right) & =n(\tilde{\Gamma}, q) \\
& =\sum_{m=0}^{q}(t(\Gamma)-1)^{m}= \begin{cases}1 & \text { if } t(\Gamma)=1, \\
q+1 & \text { if } t(\Gamma)=2, \\
\frac{(t(\Gamma)-1)^{q+1}-1}{t(\Gamma)-2} & \text { if } t(\Gamma) \geq 3 .\end{cases}
\end{aligned}
$$

We obtain, for each $\tilde{\Gamma}$-module $V$, an exact sequence

$$
0 \longrightarrow V^{\tilde{\Gamma}, q} \longrightarrow V^{\tilde{\Gamma}, q+1} \stackrel{\mathrm{m}_{q}}{\longrightarrow}\left(V^{\tilde{\Gamma}}\right)^{n(\tilde{\Gamma}, q)},
$$

with

$$
\left(\mathrm{m}_{q} f\right)_{i}=f \mid\left(\alpha_{i(1)}-1\right) \ldots\left(\alpha_{i(q)}-1\right) .
$$

For the modular group, we have $n_{\mathrm{par}}=1, n_{\mathrm{ell}}=2$, and $g=0$, and hence $t\left(\Gamma_{\text {mod }}\right)=1$ and $n\left(\tilde{\Gamma}_{\text {mod }}, q\right)=1$ for all $q$. So in contrast to $\Gamma_{\text {mod }}$, for $\tilde{\Gamma}_{\text {mod }}$ we may hope for nontrivial higher-order automorphic forms.

\section{Maass forms with generalized weight on the universal covering group}

6A. The logarithm of the Dedekind eta function. In the Introduction, we mentioned that one of the motivating objects for the study of higher-order forms on the universal covering group is the logarithm of the Dedekind eta function. Its branch is fixed by the second of the following expressions:

$$
\log \eta(z)=\frac{\pi i z}{12}+\sum_{n=1}^{\infty} \log \left(1-e^{2 \pi i n z}\right)=\frac{\pi i z}{12}-\sum_{n=1}^{\infty} \sigma_{-1}(n) e^{2 \pi i n z},
$$

where $\sigma_{u}(n)=\sum_{d \mid n} d^{u}$. One can show that its behavior under $\Gamma_{\bmod }$ is given by

$$
\log \eta(z+1)=\log \eta(z)+\frac{\pi i}{12}, \quad \log \eta\left(-\frac{1}{z}\right)=\log \eta(z)+\frac{1}{2} \log z-\frac{\pi i}{4} .
$$

Except for the term $\frac{1}{2} \log z$, this looks like a second-order holomorphic modular form of weight 0 . In the next few sections, we make this precise by generalizing the concept of weight of Maass forms, and replacing the group $\Gamma_{\text {mod }}$ by the discrete subgroup $\tilde{\Gamma}_{\text {mod }}$ of the universal covering group of $\mathrm{SL}_{2}(\mathbb{R})$, using the notation we introduced in the last section.

We first define the following function on $\mathfrak{H} \times \mathbb{R}$ :

$$
L(z, \vartheta)=\frac{1}{2} \log y+2 \log \eta(z)+i \vartheta .
$$

With (6-2), we check easily that $L(\gamma(z, \vartheta))=L(z, \vartheta)+i \alpha(\gamma)$ for $\gamma=t$ and $\gamma=s$, where $\alpha: \tilde{\Gamma}_{\text {mod }} \rightarrow(\pi / 6) \mathbb{Z}$ is the group homomorphism at the end of Section 5B. 
Thus, $L$ has the transformation behavior of a second-order invariant in the functions on $\tilde{G}$ for the action by left translation.

Routine computations show that $L$ satisfies $\boldsymbol{E}^{-} L=0, \boldsymbol{W} L=i$, and $\omega L=\frac{1}{2}$.

6B. General Maass forms on the universal covering group. The considerations on the function $L$ on $\tilde{G}$ induced by the logarithm of the eta functions lead us to the definition of Maass forms on $\tilde{G}$.

We first establish appropriate notions of weight and holomorphicity. We say that a function $f$ on $\tilde{G}$ has (strict) weight $r \in \mathbb{C}$ if $f(z, \vartheta)=e^{i r \vartheta} f(z, 0)$. Such a function is completely determined by the function $f_{r}(z)=f(z, 0)$ on $\mathfrak{H}$ and satisfies $\boldsymbol{W} f=\operatorname{irf}$.

The left translation of $f$ by $\tilde{g}$, with $g=\left(\begin{array}{ll}a & b \\ c & d\end{array}\right) \in \mathrm{SL}_{2}(\mathbb{Z})$, induces an action $\mid$ of $\tilde{G}$ on the space of functions of strict weight on $\tilde{G}$. On the other hand, $\tilde{G}$ acts on the space of corresponding functions $f_{r}$ on $\mathfrak{H}$ via

$$
f_{r} \mid \tilde{g}(z)=e^{-i r \arg (c z+d)} f_{r}\left(\frac{a z+b}{c z+d}\right) .
$$

The latter action corresponds to (4-7) when $r \in \mathbb{Z}$. In general, this is an action of $\tilde{G}$, not of $\mathrm{SL}_{2}(\mathbb{R})$. The map $f \mapsto f_{r}$ defined above on the space of functions of strict weight is then equivariant in terms of these actions.

Many important functions on $\tilde{G}$, such as $L$, are not eigenfunctions of the operator $\boldsymbol{W}$, but they are annihilated by a power of $\boldsymbol{W}$. This suggests the following definition.

Definition 6.1. An $f \in C^{\infty}(\tilde{G})$ has generalized weight $r \in \mathbb{C}$ if $(\boldsymbol{W}-i r)^{n} f=0$ for some $n \in \mathbb{N}$.

Thus, $L$ and all its powers have generalized weight 0 .

Next, holomorphy of $F_{r}=y^{-r / 2} f_{r}$ corresponds to the property $\boldsymbol{E}^{-} f=0$.

Definition 6.2. We call any differentiable function $f$ on $\tilde{G}$ holomorphic (resp. antiholomorphic) if $\boldsymbol{E}^{-} f=0$ (resp. $\boldsymbol{E}^{+} f=0$ ). We call any twice differentiable function $f$ on $\tilde{G}$ harmonic if it satisfies $\omega f=0$.

Note that, for functions of nonzero weight, this definition of harmonicity does not correspond to the use of the word in "harmonic weak Maass forms" in [Bruinier et al. 2008], for example.

With these definitions, we set:

Definition 6.3. Let $k, \lambda \in \mathbb{C}$. Let $\tilde{\Gamma}$ be a discrete cofinite subgroup of $\tilde{G}$.

i) The space $\tilde{\mathscr{E}}_{k}(\tilde{\Gamma}, \lambda)$ consists of the smooth functions $f: \mathfrak{H} \times \mathbb{R} \rightarrow \mathbb{C}$ satisfying:

a) (eigenfunction Casimir operator) $\omega f=\lambda f$.

b) (generalized weight) $(\boldsymbol{W}-i k)^{n} f=0$ for some $n \in \mathbb{N}$. 
c) (exponential growth) There exists $a \in \mathbb{R}$ such that for all compact sets $X$ and $\Theta \subset \mathbb{R}$ and for all cusps $\kappa$ of $\tilde{\Gamma}$, we have

$$
f\left(\tilde{g}_{\kappa}(x+i y, \vartheta)\right)=\mathrm{O}\left(e^{a y}\right)
$$

as $y \rightarrow \infty$ uniformly in $x \in X$ and $\vartheta \in \Theta$.

ii) We set

$$
\tilde{E}_{k}(\tilde{\Gamma}, \lambda):=\tilde{\mathscr{E}}_{k}(\tilde{\Gamma}, \lambda)^{\tilde{\Gamma}}
$$

(where $\tilde{\Gamma}$ acts by left translation). The elements of $\tilde{E}_{k}(\tilde{\Gamma}, \lambda)$ are called Maass forms on $\tilde{G}$ of generalized weight $k$ and eigenvalue $\lambda$ for $\tilde{\Gamma}$.

The space $\tilde{E}_{r}(\tilde{\Gamma}, \lambda)$ is infinite-dimensional. Further, since $\omega$ and $\boldsymbol{W}$ commute with left translations in $\tilde{G}$, the space $\tilde{\mathscr{E}}_{k}(\tilde{\Gamma}, \lambda)$ is invariant under left translation by elements of $\tilde{\Gamma}$.

When $k \in 2 \mathbb{Z}$, the space $E_{k}(\Gamma, \lambda)$ can be identified with $\tilde{E}_{k}(\tilde{\Gamma}, \lambda)$. We prove the following slightly stronger statement.

Theorem 6.4. Let $\tilde{\Gamma}$ be a cofinite discrete subgroup of $\tilde{G}$, and let $k, \lambda \in \mathbb{C}$. If $\tilde{\mathscr{E}}_{k}(\tilde{\Gamma}, \lambda)^{\tilde{Z}}$ contains a nonzero element $f$, then $k \in 2 \mathbb{Z}$ and $\partial_{\vartheta} f(z, \vartheta)=i k f(z, \vartheta)$.

If $k \in 2 \mathbb{Z}$, then the elements $f \in \tilde{E}_{k}(\tilde{\Gamma}, \lambda)$ correspond bijectively to the Maass forms $F \in E_{k}(\Gamma, \lambda)$ by

$$
f(z, \vartheta)=y^{k / 2} F(z) e^{i k \vartheta} .
$$

So the condition of $\tilde{Z}$-invariance implies that the weight $k$ is even, and that the weight is strict, that is, condition b) holds with $n=1$.

Proof of Theorem 6.4. Any smooth function $f \in C^{\infty}(\mathfrak{H} \times \mathbb{R})$ satisfying b) in Definition 6.3 can be written in the form $f(z, \vartheta)=\sum_{j=0}^{n-1} \varphi_{j}(z) e^{i k \vartheta} \vartheta^{j}$, with $\varphi_{j} \in C^{\infty}(\mathfrak{H})$.

If such a function is left-invariant under $\tilde{Z}$, then the action of $k(\pi m) \in \tilde{Z} \subset \tilde{\Gamma}$ implies, for each $m \in \mathbb{Z}$,

$$
e^{\pi i k m} \sum_{j} \varphi_{j}(z) e^{i k \vartheta}(\vartheta+\pi m)^{j}=\sum_{j} \varphi_{j}(z) e^{i k \vartheta} \vartheta^{j}, \quad \text { for all } m \in \mathbb{Z} .
$$

With induction, this gives $k \in 2 \mathbb{Z}$ and $\varphi_{j}=0$ for $j \geq 1$, and hence $f(z, \vartheta)=\varphi_{0}(z) e^{i k \vartheta}$. Moreover, the stronger condition $f \in \tilde{E}_{k}(\tilde{\Gamma}, \lambda)=\tilde{\mathscr{E}}_{k}(\tilde{\Gamma}, \lambda)^{\tilde{\Gamma}}$ can be checked to be equivalent to $F_{k} \in E_{k}(\Gamma, \lambda)$ for $F_{k}(z)=y^{-k / 2} f(z, 0)$.

We have the following generalization of Theorem 4.2.

Theorem 6.5. Let $\tilde{\Gamma}$ be a cofinite discrete subgroup of $\tilde{G}$ with cusps. Then the $\tilde{\Gamma}$-module $\tilde{\mathscr{E}}_{k}(\tilde{\Gamma}, \lambda)$ is maximally perturbable for each $k \in 2 \mathbb{Z}$ and each $\lambda \in \mathbb{C}$. 
In Section 8 we will prove this theorem. In this section we will show that it implies the corresponding result for $E_{k}(\Gamma, \lambda)$. We first give some facts that are of more general interest.

The map identifying $E_{k}(\Gamma, \lambda)$ and $\tilde{E}_{k}(\tilde{\Gamma}, \lambda)$ can be extended to an isomorphism

$$
\mu: \mathscr{E}_{k}(\Gamma, \lambda) \rightarrow \tilde{\mathscr{E}}_{k}(\tilde{\Gamma}, \lambda)^{\tilde{Z}} .
$$

Since the center $\tilde{Z}$ of $\tilde{\Gamma}$ acts trivially on $\tilde{\mathscr{E}}_{k}(\tilde{\Gamma}, \lambda)^{\tilde{Z}}$, it can be considered as a $\Gamma$ module. With this interpretation, we obtain an identification of the $\Gamma$-modules $\mathscr{E}_{k}(\Gamma, \lambda)$ and $\tilde{\mathscr{E}}_{k}(\tilde{\Gamma}, \lambda)^{\tilde{Z}}$. Specifically, for $F \in \mathscr{E}_{k}(\Gamma, \lambda), g \in \tilde{\mathscr{E}}_{k}(\tilde{\Gamma}, \lambda)^{\tilde{Z}}$ we have

$$
\begin{array}{rlrl}
(\mu f)(z, \vartheta) & =y^{k / 2} F(z) e^{i k \vartheta}, & \\
\left(\mu^{-1} g\right)(z) & =y^{-k / 2} g(z, 0), & & \\
\mu\left(\left.F\right|_{k} \gamma\right) & =\mu(F) \mid v(\gamma) & & (\gamma \in \Gamma), \\
\mu^{-1}(g \mid \tilde{Z} \delta) & =\left.\mu^{-1}(g)\right|_{k} \nu^{-1}(\tilde{Z} \delta) & (\delta \in \tilde{\Gamma}),
\end{array}
$$

where $v$ denotes the isomorphism identifying $\Gamma$ with $\tilde{Z} \backslash \tilde{\Gamma}$.

Proposition 6.6. Let $\Gamma$ be a cofinite discrete subgroup of $G$ with cusps, and let $\tilde{\Gamma}=\operatorname{pr}^{-1} \Gamma$. If the $\tilde{\Gamma}$-module $V$ is maximally perturbable, then the subspace $V^{\tilde{Z}}$, considered as a $\Gamma$-module, is maximally perturbable.

Proof. The projection pr: $\tilde{\Gamma} \rightarrow \Gamma$ induces linear maps pr: $\mathbb{C}[\tilde{\Gamma}] \rightarrow \mathbb{C}[\Gamma]$ between the group rings, $\mathrm{pr}: I_{\tilde{\Gamma}} \rightarrow I_{\Gamma}$ between the augmentation ideals, and pr: $I_{\tilde{\Gamma}}^{q+1} \backslash I_{\tilde{\Gamma}}^{q} \rightarrow I_{\Gamma}^{q+1} \backslash I_{\Gamma}^{q}$ for all $q \in \mathbb{N}$. Since $\operatorname{pr}\left(A_{i}\right)=\alpha_{\boldsymbol{i}}$, on the basis elements $\boldsymbol{b}_{\tilde{\Gamma}}(\boldsymbol{i})$ in Proposition 5.1 and $\boldsymbol{b}_{\Gamma}(\boldsymbol{i})$ in (3-8), we have for $\tilde{\Gamma}$ - $q$-tuples:

$$
\operatorname{pr} \boldsymbol{b}_{\tilde{\Gamma}}(\boldsymbol{i})= \begin{cases}\boldsymbol{b}_{\Gamma}(\boldsymbol{i}) & \text { if } \boldsymbol{i}(l)<t(\Gamma) \text { for } l=1, \ldots, q, \\ 0 & \text { if } \boldsymbol{i}(q)=t(\Gamma) .\end{cases}
$$

This means that we have the commutative diagram

$$
\begin{aligned}
& 0 \longrightarrow V^{\tilde{\Gamma}, q} \longrightarrow V^{\tilde{\Gamma}, q+1} \stackrel{\mathrm{m}_{q}}{\longrightarrow} \operatorname{hom}\left(I_{\tilde{\Gamma}}^{q+1} \backslash I_{\tilde{\Gamma}}^{q}, V^{\tilde{\Gamma}}\right) \longrightarrow 0 \\
& 0 \longrightarrow\left(V^{\tilde{Z}}\right)^{\Gamma, q} \longrightarrow\left(V^{\tilde{Z}}\right)^{\Gamma, q+1} \stackrel{\mathrm{m}_{q}}{\longrightarrow} \operatorname{hom}\left(I_{\Gamma}^{q+1} \backslash I_{\Gamma}^{q},\left(V^{\tilde{Z}}\right)^{\Gamma}\right),
\end{aligned}
$$

where the vertical arrow sends $f: I_{\Gamma}^{q+1} \backslash I_{\Gamma}^{q} \rightarrow\left(V^{\tilde{Z}}\right)^{\Gamma}=V^{\tilde{\Gamma}}$ to $\tilde{f}: I_{\tilde{\Gamma}}^{q+1} \backslash I_{\tilde{\Gamma}}^{q} \rightarrow V^{\tilde{\Gamma}}$ such that $\tilde{f}\left(\boldsymbol{b}_{\tilde{\Gamma}}(\boldsymbol{i})\right)=f\left(\boldsymbol{b}_{\Gamma}(\boldsymbol{i})\right)$ if $\boldsymbol{i} \in\{1, \ldots, t(\Gamma)-1\}^{q}$, and $\tilde{f}\left(\boldsymbol{b}_{\tilde{\Gamma}}(\boldsymbol{i})\right)=0$ otherwise.

We want to write a given $f: I_{\Gamma}^{q+1} \backslash I_{\Gamma}^{q} \rightarrow\left(V^{\tilde{Z}}\right)^{\Gamma}$ as $\mathrm{m}_{q} v_{0}$ with $v_{0} \in\left(V^{\tilde{Z}}\right)^{\Gamma, q+1}$. By assumption, there is an element $v \in V^{\tilde{\Gamma}, q+1}$ such that $\mathrm{m}_{q} v=\tilde{f}$. If $v \mid(\zeta-1)=0$, then $v \in V^{\tilde{\Gamma}, q+1} \cap V^{\tilde{Z}}=\left(V^{\tilde{Z}}\right)^{\Gamma, q+1}$, and we are done. 
Suppose that $w=v \mid(\zeta-1) \neq 0$. Take $r \in[1, q]$ minimal such that $w \in V^{\tilde{\Gamma}, r}$. We will show that we can replace $v$ by another element $v_{1} \in v+V^{\tilde{\Gamma}, q}$ with $v_{1} \mid(\zeta-1) \in$ $V^{\tilde{\Gamma}, r_{1}}$ and $r_{1}<r$. Repeating this process brings us eventually to $v_{j} \mid(\zeta-1)=0$. For this $v_{j}$, we will have $\mathrm{m}_{q} v_{j}=\tilde{f}$ and $v_{j} \mid(\zeta-)=0$, which, according to the remark of the last paragraph, suffices to prove the proposition.

From

$$
\begin{aligned}
w \mid\left(\gamma_{1}-1\right) \ldots\left(\gamma_{q-1}-1\right) & =v \mid\left(\gamma_{1}-1\right) \ldots\left(\gamma_{q-1}-1\right)(\zeta-1) \\
& =\tilde{f}\left(\gamma_{1}, \ldots, \gamma_{q-1}, \zeta\right)=0,
\end{aligned}
$$

we conclude that $r \leq q-1$. Define $\tilde{g} \in \operatorname{hom}\left(I_{\tilde{\Gamma}}^{r+1} \backslash I_{\tilde{\Gamma}}^{r}, V^{\tilde{\Gamma}}\right)$ by

$$
\tilde{g}\left(\boldsymbol{b}_{\tilde{\Gamma}}(\boldsymbol{j})\right)=w \mid\left(\alpha_{\boldsymbol{j}(1)}-1\right) \ldots\left(\alpha_{\boldsymbol{j}(r-1)}-1\right)
$$

if the $\tilde{\Gamma}$-r-tuple $\boldsymbol{j}$ satisfies $\boldsymbol{j}(r)=t(\Gamma)$, and $\tilde{g}\left(\boldsymbol{b}_{\tilde{\Gamma}}(\boldsymbol{j})\right)=0$ otherwise. There is $u \in V^{\tilde{\Gamma}, r+1} \subset V^{\tilde{\Gamma}, q}$ with $\mathrm{m}_{r} u=\tilde{g}$. We take $v_{1}=v-u \in v+V^{\tilde{\Gamma}, q}$. We check that for all $\tilde{\Gamma}$ - $(r-1)$-tuples $\boldsymbol{j}$,

$$
\begin{aligned}
v_{1} \mid(\zeta-1) & \left(\alpha_{j(1)}-1\right) \ldots\left(\alpha_{j(r-1)}-1\right) \\
& =w\left|\left(\alpha_{j(1)}-1\right) \ldots\left(\alpha_{j(r-1)}-1\right)-u\right|\left(\alpha_{j(1)}-1\right) \ldots\left(\alpha_{j(r-1)}-1\right)(\zeta-1) \\
& =0
\end{aligned}
$$

This shows that $v_{1} \mid(\zeta-1)$ has order less than $r$.

Proof of Theorem 4.2. From Theorem $6.5, V=\tilde{\mathscr{E}}_{k}\left(\tilde{\Gamma}, \lambda_{k}\right)$ is maximally perturbable. Therefore, by Proposition 6.6, the space $\tilde{\mathscr{E}}_{k}\left(\tilde{\Gamma}, \lambda_{k}\right) \tilde{Z} \cong \mathscr{E}_{k}\left(\Gamma, \lambda_{k}\right)$ is maximally perturbable too.

This proof illustrates the fact that, for groups with cusps, there are really more higher-order forms with generalized weight than with strict weight: the basis in Proposition 5.1 is for all such discrete groups larger than the corresponding basis in Section 3B.

\section{C. Holomorphic forms on the universal covering group.}

Definition 6.7. For $k \in 2 \mathbb{Z}$, we define $\mathscr{H}_{k}(\tilde{\Gamma})$ as the space of elements of $C^{\infty}(\mathfrak{H} \times \mathbb{R})$ that satisfy:

i) (Holomorphy): $\boldsymbol{E}^{-} f=0$.

ii) (Generalized weight): $(\boldsymbol{W}-i k)^{n} f=0$, for some $n \in \mathbb{N}$.

iii) (Exponential growth): as described in condition c) in Definition 6.3.

This is a $\tilde{\Gamma}$-module for the action by left translation. We denote by $\mathscr{H}_{k}^{p}(\tilde{\Gamma})$ (resp. $\left.\mathscr{H}_{k}^{c}(\tilde{\Gamma})\right)$ the space of $f \in \mathscr{H}_{k}(\tilde{\Gamma})$ satisfying $f\left(\tilde{g}_{\kappa}(x+i y, \vartheta)\right)=\mathrm{O}\left(y^{C}\right)$ for some $C \in \mathbb{R}\left(\right.$ resp. $f\left(\tilde{g}_{\kappa}(x+i y, \vartheta)\right)=\mathrm{O}\left(e^{a y}\right)$ for some $\left.a<0\right)$ instead of (6-4).

We will prove: 
Theorem 6.8. Let $\tilde{\Gamma}$ be a cofinite discrete subgroup of $\tilde{G}$ with cusps. Then the $\tilde{\Gamma}$-module $\mathscr{H}_{k}(\tilde{\Gamma})$ is maximally perturbable for each $k \in 2 \mathbb{Z}$.

Proof of Theorem 4.3. As in the case of general Maass forms, we can show that, for $k \in 2 \mathbb{Z}, \mathscr{C}_{k}^{\text {hol }}\left(\Gamma, \lambda_{k}\right) \cong \mathscr{H}_{k}(\tilde{\Gamma})^{\tilde{Z}}$. Then Proposition 6.6 implies Theorem 4.3.

Second-order forms and derivatives of $\boldsymbol{L}$-functions. With this definition, $L$ is a second-order invariant belonging to $\mathscr{H}_{0}\left(\tilde{\Gamma}_{\text {mod }}\right)^{\tilde{\Gamma}_{\text {mod }}, 2}$. (Incidentally, this example shows that, for generalized weight $k$, the space $\mathscr{H}_{k}(\tilde{\Gamma})$ need not be contained in $\tilde{\mathscr{E}}_{k}\left(\tilde{\Gamma}, \lambda_{k}\right)$.)

Based on $L$, we can construct a second-order form which is related to derivatives of classical modular forms. Specifically, for positive integer $N$, denote by $G_{N}$ the group generated by $\tilde{g}, g \in\left\langle\Gamma_{0}(N), W_{N}\right\rangle$, where

$$
W_{N}:=\left(\begin{array}{cc}
0 & -\sqrt{N}^{-1} \\
\sqrt{N} & 0
\end{array}\right) .
$$

Set

$$
L_{1}(z, \vartheta)=L(z, \vartheta)+L(N z, \vartheta)
$$

Using the transformation law for $L$ and the identity $\left(\begin{array}{cc}N & 0 \\ 0 & 1\end{array}\right)\left(\begin{array}{cc}a & b \\ N c & d\end{array}\right)=\left(\begin{array}{cc}a & N b \\ c & d\end{array}\right)\left(\begin{array}{cc}N & 0 \\ 0 & 1\end{array}\right)$, a routine calculation implies that, for some $\beta \in \operatorname{Hom}\left(G_{N}, \mathbb{C}\right)$,

$$
L_{1}(\gamma(z, \vartheta))=L_{1}(z, \vartheta)+i \beta(\gamma), \quad \text { for all } \gamma \in G_{N} .
$$

Now let $f$ be in the space $S_{2}\left(G_{N}\right)$ of cusp forms of weight 2 for $G_{N}$. An example of such an $f$ is a weight-2 newform for $\Gamma_{0}(N)$ of which the $L$-function $L_{f}(s)$ vanishes at 1 , because then $f\left(W_{N} w\right) d\left(W_{N} w\right)=f(w) d w$. For all $\vartheta \in \mathbb{R}$,

$$
\begin{aligned}
\int_{0}^{\infty} f(i y) L_{1}(i y, \vartheta) d i y & =-\int_{W_{N} 0}^{W_{N} \infty} f(i y) L_{1}(i y, \vartheta) d i y \\
& =-\int_{0}^{\infty} f\left(W_{N} i y\right) L_{1}\left(W_{N} i y, \vartheta\right) d\left(W_{N} i y\right) \\
& =-\int_{0}^{\infty} f(i y) L_{1}\left(W_{N} i y, \vartheta\right) d i y .
\end{aligned}
$$
Since $L_{1}(z, \vartheta+x)=L_{1}(z, \vartheta)+2 i x$ and $L_{f}(1)=2 \pi \int_{0}^{\infty} f(i y) d y=0$, our integral
is independent of $\vartheta$. It further equals

$$
\begin{aligned}
-\int_{0}^{\infty} f(i y) L_{1}\left(\tilde{W}_{N}(i y, 0)\right) d i y & =-\int_{0}^{\infty} f(i y)\left(L_{1}(i y, 0)+i \beta\left(\tilde{W}_{N}\right)\right) d i y \\
& =-\int_{0}^{\infty} f(i y) L_{1}(i y, 0) d i y .
\end{aligned}
$$


Therefore, $\int_{0}^{\infty} f(i y) L_{1}(i y, 0) d y=-\int_{0}^{\infty} f(i y) L_{1}(i y, 0) d y$, that is,

$$
\int_{0}^{\infty} f(i y) L_{1}(i y, 0) d y=0
$$

and hence

$$
\int_{0}^{\infty} f(i y) \log y d y+2 \int_{0}^{\infty} f(i y) u(i y) d y=0
$$

where $u(z):=\log (\eta(z))+\log (\eta(N z))$. From this we see that, since $L_{f}^{\prime}(s)=$ $2 \pi \int_{0}^{\infty} f(i y) \log (y) d y$, we can retrieve, from an alternative perspective, the formula

$$
L_{f}^{\prime}(1)=-4 \pi \int_{0}^{\infty} f(i y) u(i y) d y
$$

first derived in [Goldfeld 1995].

Thus, Goldfeld's expression of $L_{f}^{\prime}(1)$ is equivalent to the orthogonality of $L_{1} \in$ $\mathscr{H}_{0}^{p}\left(G_{N}\right)^{G_{N}, 2}$ to the space $S_{2}\left(G_{N}\right) \hookrightarrow \mathscr{H}_{2}^{c}\left(G_{N}\right)^{G_{N}}$ in terms of the pairing

$$
\langle\cdot, \cdot\rangle: \mathscr{H}_{2}^{c}\left(G_{N}\right)^{G_{N}} \times \mathscr{H}_{0}^{p}\left(G_{N}\right)^{G_{N}, 2} \rightarrow \mathbb{C}
$$

defined by

$$
\langle g, h\rangle=\int_{0}^{\infty} g(i y, 0) h(i y, 0) \frac{d y}{y} .
$$

6D. Examples of higher-order forms for the full modular group. Theorems 6.5 and 6.8 show that there are perturbations of 1 for the full original $\tilde{\Gamma}_{\text {mod }}$ of $\mathrm{SL}_{2}(\mathbb{Z})$ in the universal covering group. Since $t\left(\Gamma_{\bmod }\right)=1$, all these perturbations are commutative (see (5-10)).

1) The function $L$ can lead to second-order harmonic perturbations of 1 . Specifically, although $L \notin \tilde{\mathscr{E}}_{0}(0)^{\tilde{\Gamma}, 2}$ (because $\omega L=\frac{1}{2}$ ), the imaginary part $\operatorname{Im} L:(z, \vartheta) \mapsto$ $2 \operatorname{Im} \log \eta(z)+\vartheta$ is harmonic, has second order, and corresponds to the linear form $\alpha \in \operatorname{Mult}^{1}\left(\tilde{\Gamma}_{\text {mod }}, \mathbb{C}\right)$. It has generalized weight 0 , and it is not holomorphic.

2) Set $\chi_{r}=e^{i r \alpha}, r \in \mathbb{C}$, where $\alpha \in \operatorname{hom}\left(\tilde{\Gamma}_{\text {mod }}, \mathbb{C}\right)$ is given by $\alpha(n(1))=\pi / 6$ and $\alpha(k(\pi / 2))=\pi / 2$. The family

$$
r \mapsto e^{r L(z, \vartheta)}=y^{r / 2} \eta(z)^{2 r} e^{i r \vartheta}
$$

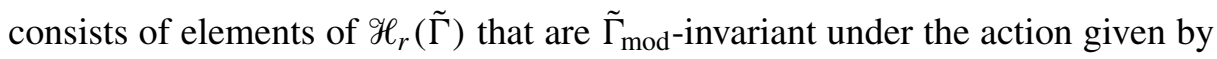

$$
(f \mid \gamma)(z)=f(\gamma z) \overline{\chi_{r}(\gamma)}
$$

By Proposition 4.4 , for $k \geq 1$ the derivative

$$
\left.\partial_{r}^{k} e^{r L(z, \vartheta)}\right|_{r=0}=L(z, \vartheta)^{k}
$$


is a holomorphic perturbation of 1 of order $k+1$. The corresponding element of $\operatorname{Mult}^{k}\left(\tilde{\Gamma}_{\text {mod }}, \mathbb{C}\right)$ is $i^{k} k ! \alpha^{\otimes k}$.

3) It is possible to obtain a more or less explicit description of a harmonic perturbation of 1 of order 3 . We sketch how this can be done with the meromorphic continuation of the Eisenstein series in weight and spectral parameter jointly. This family is studied in [Bruggeman 1986]. In that work, automorphic forms are described as functions on $\mathfrak{H}$ transforming according to a multiplier system of $\Gamma_{\text {mod }}$. These correspond to functions on $\tilde{G}$ that transform according to a character of $\tilde{\Gamma}_{\text {mod }}$. Carrying out the reformulation, we can rephrase $\$ 2.18$ in [Bruggeman 1986] as stating that there is a meromorphic family of Maass forms on $U \times \mathbb{C}$, where $U$ is some neighborhood of $(-12,12)$ in $\mathbb{C}$. We retrieve the exact family studied in [Bruggeman 1986] by considering $z \mapsto E(r, s ; z, 0)$. For each $(r, s) \in U \times \mathbb{C}$ at which $E$ is not singular, it is an automorphic form of weight $r$ for the character $\chi_{r}=e^{i r \alpha}$ of $\tilde{\Gamma}_{\bmod }$ with eigenvalue $\lambda_{s}=\frac{1}{4}-s^{2}$. It is a meromorphic family of automorphic forms on $\tilde{\Gamma}_{\text {mod }}$ with character $\chi_{r}$ with a Fourier expansion of the form

$$
E(r, s)=\mu_{r}\left(\frac{r}{12}, s\right)+C_{0}(r, s) \mu_{r}\left(\frac{r}{12},-s\right)+\sum_{n \neq 0} C_{n}(r, s) \omega_{r}\left(n+\frac{r}{12}, s\right),
$$

where the $C_{n}(r, s)$ are meromorphic functions, and where we use the following notations:

$$
\begin{aligned}
& \omega_{r}(v, s ; z, \vartheta)=e^{2 \pi i v x} W_{r} \operatorname{Sign}(\operatorname{Re} v) / 2, s(4 \pi v \operatorname{Sign}(\operatorname{Re} v) y) e^{i r \vartheta}, \\
& \mu_{r}(v, s ; z, \vartheta)=e^{2 \pi i v z} y^{1 / 2+s}{ }_{1} F_{1}\left(\frac{1}{2}+s-\frac{r}{2} ; 1+2 s ; 4 \pi v y\right) e^{i r \vartheta} .
\end{aligned}
$$

This family and its Fourier coefficient $C_{0}$ satisfy the following functional equations:

$$
\begin{aligned}
E(r,-s) & =C_{0}(r,-s) E(r, s), \\
E(r, s ;-x+i y,-\vartheta) & =E(-r, s ; x+i y, \vartheta) .
\end{aligned}
$$

Further, the restriction of this family to the (complex) line $r=0$ exists, and gives a meromorphic family of automorphic forms depending on one parameter $s$. This is a family of weight 0 , so it does not depend on the parameter $\vartheta$ on $\tilde{G}$. The resulting family on $\mathfrak{H}$ is the meromorphic continuation of the Eisenstein series for $\Gamma_{\text {mod }}$ in weight 0 , with Fourier expansion

$$
\begin{aligned}
E(0, s)=\mu_{0}(0, s)+\frac{\sqrt{\pi} \Gamma(s) \zeta(2 s)}{\Gamma\left(s+\frac{1}{2}\right) \zeta(2 s+1)} \mu_{0}(0,-s) & \\
& +\frac{\pi^{s+1 / 2}}{\Gamma\left(s+\frac{1}{2}\right) \zeta(2 s+1)} \sum_{n \neq 0} \frac{\sigma_{2 s}(|n|)}{|n|^{s+1 / 2}} \omega_{0}(n, s),
\end{aligned}
$$


where

$$
\begin{aligned}
& \mu_{0}(0, s ; z, \vartheta)=y^{1 / 2+s} \\
& \omega_{0}(n, s ; z, \vartheta)=e^{2 \pi i n x} W_{0, s}(4 \pi|n| y)=e^{2 \pi i n x} 2|n|^{1 / 2} K_{s}(2 \pi|n| y) .
\end{aligned}
$$

At $\left(0,-\frac{1}{2}\right)$, the family $E$ is holomorphic in both variables $r$ and $s$, with a constant as its value at $\left(0,-\frac{1}{2}\right)$; this is a consequence of Proposition 6.5 ii) in [Bruggeman 1986]. So in principle, we obtain higher-order harmonic perturbations of 1 by differentiating $r \mapsto E\left(r,-\frac{1}{2}\right)$. Here we encounter the problem that we have an explicit Fourier expansion (6-13) only for $E(0, s)$, and thus we cannot describe the derivatives in the direction of $r$ directly. To overcome this problem, we use the fact that for $r$ near 0 , we have

$$
\begin{aligned}
& E\left(r,-\frac{1-r}{2} ; z, \vartheta\right)=H_{r}(z, \vartheta)=e^{r L(z, \vartheta)}, \\
& E\left(r,-\frac{1+r}{2} ; z, \vartheta\right)=H_{-r}(-\bar{z},-\vartheta)=e^{-r \overline{L(z, \vartheta)}} .
\end{aligned}
$$

The proof of the first equality is contained in 6.10 in [Bruggeman 1986]. The second one follows from the second functional equation in (6-12). Now we use the Taylor expansion of $E$ of degree 2 at $(r, s)=\left(0,-\frac{1}{2}\right)$ :

$E(r, s)$

$=1+r A_{1,0}+\left(s+\frac{1}{2}\right) A_{0,1}+\frac{1}{2} r^{2} A_{2,0}+r\left(s+\frac{1}{2}\right) A_{1,1}+\frac{1}{2}\left(s+\frac{1}{2}\right)^{2} A_{0,2}+\ldots$.

By Proposition 4.4, the coefficients $A_{1,0}$ and $A_{2,0}$ are harmonic perturbations of 1 of order 2 and 3, respectively. From (6-14), we obtain the following results:

$$
\begin{aligned}
A_{1,0} & =i \operatorname{Im} L, & A_{0,1} & =2 \operatorname{Re} L, \\
A_{2,0}+\frac{1}{4} A_{0,2} & =\operatorname{Re} L^{2}, & A_{1,1} & =i \operatorname{Im} L^{2} .
\end{aligned}
$$

This confirms that $\operatorname{Im} L$ is a second-order harmonic perturbation of 1. Differentiation

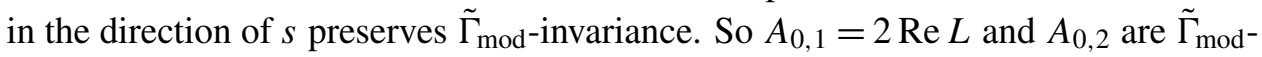
invariant. However, these functions are not in the kernel of $\omega$.

Thanks to the identity $A_{2,0}+\frac{1}{4} A_{0,2}=\operatorname{Re} L^{2}$, to determine the third-order harmonic perturbation $A_{2,0}$ it suffices to explicitly compute $A_{0,2}$, because $\operatorname{Re} L^{2}$ is known in a fairly explicit way. The function $A_{0,2}$ can be obtained as the coefficient of $\frac{1}{2}\left(s+\frac{1}{2}\right)^{2}$ in the Taylor expansion of $E(0, s)$ at $s=-\frac{1}{2}$. As a by-product of this computation, we will also obtain the $\tilde{\Gamma}_{\text {mod-invariant function } A_{0,1} \text { as the coefficient }}$ of $s+\frac{1}{2}$ in the same expansion.

We examine each term in the Fourier expansion (6-13) separately. We use the functional equation of the Riemann zeta function and its expansion at the point 0 . 
We also use an integral representation of the Whittaker function $W_{0, s}$. This leads to

$$
\begin{aligned}
A_{0,1}(z, 0)= & \log y-\frac{\pi}{3} y-2 \sum_{n \geq 1} \sum_{d \mid n} \frac{1}{d}\left(q^{n}+\bar{q}^{n}\right) \\
= & 2 \operatorname{Re}\left(\frac{1}{2} \log y+\frac{\pi i}{6} z-\sum_{n=1}^{\infty} \sigma_{-1}(n) q^{n}\right)=2 \operatorname{Re} L(z, 0), \\
A_{0,2}(z, 0)= & (\log y)^{2}+\left(8 b_{1}-\frac{4 \pi a_{0}}{3}+\frac{2 \pi}{3} \log y\right) y \\
& +\sum_{n=1}^{\infty}\left(-4 a_{0} \sigma_{-1}(n)\left(q^{n}+\bar{q}^{n}\right)+2 \sigma_{-1}(n)\left(q^{-n}+\bar{q}^{-n}\right) \Gamma(0,4 \pi n y)\right. \\
& \left.-2\left(q^{n}+\bar{q}^{n}\right) \sum_{d \mid n} \frac{\log \left(d^{2} / n\right)}{d}\right),
\end{aligned}
$$

with the notation $q=e^{2 \pi i z}$.

A remarkable aspect of this computation is that we have used an explicit computation of the derivatives of the Eisenstein series in weight 0 to compute the second derivative in the $r$-direction of the more complicated Eisenstein family in two variables. The basic observation is (6-14), which shows that the Eisenstein family has easy derivatives in two directions. The Taylor expansion of $E$ at $\left(0,-\frac{1}{2}\right)$ has three monomials in order 2. So it suffices to compute a second-order derivative in one more direction to get hold of all terms. Higher-order terms in the Taylor expansion have too many monomials for this method to work. We do not know how to compute all harmonic perturbations of 1 of higher order.

\section{Higher-order Fourier expansions}

This section is needed for the constructions on which the proofs of Theorems 6.5 and 6.8 are based, but it is also of independent interest. It provides a higher-order analogue of the classical Fourier expansions.

7A. Fourier expansion of Maass forms. If $f$ is in $\tilde{E}_{r}(\tilde{\Gamma}, \lambda)$, then for each cusp $\kappa$ of $\Gamma$ there is a Fourier expansion

$$
f\left(\tilde{g}_{\kappa} g\right)=\sum_{\nu} F_{\kappa, \nu} f(g), \quad F_{\kappa, \nu} f(g)=\int_{0}^{1} e^{-2 \pi i v x} f\left(\tilde{g}_{\kappa} n(x) g\right) d x,
$$

where $v$ runs through a class in $\mathbb{C} \bmod \mathbb{Z}$ determined by $\chi$ and the cusp $\kappa$. The function $F_{\nu} f$ satisfies $F_{\kappa, v} f(z, \vartheta)=e^{2 \pi i v x} F_{\kappa, v} f(i y, 0) e^{i r \vartheta}$ and $\omega F_{\kappa, v} f=\lambda F_{\kappa, v} f$.

For each given $v, r$, and $s$, set

$$
\mathcal{W}_{r}(\nu, s):=\left\{f: \tilde{G} \rightarrow \mathbb{C} ; \omega f=\left(\frac{1}{4}-s^{2}\right) f \text { and } f(z, \theta)=e^{2 \pi i v x+i r \theta} f(i y, 0)\right\} .
$$


Because of the second relation in the definition, $f \in W_{r}(\nu, s)$ can be thought of as a function of $y$. Therefore, the space $\mathcal{W}_{r}(\nu, s)$ is isomorphic to the space of $f: \mathbb{R} \rightarrow \mathbb{C}$ satisfying

$$
-y^{2} h^{\prime \prime}(y)+\left(4 \pi^{2} v^{2} y^{2}-2 \pi v r y-\frac{1}{4}+s^{2}\right) h(y)=0 .
$$

It is convenient to write $\lambda=\lambda_{s}=\frac{1}{4}-s^{2}$ with $s \in \mathbb{C}$. We can choose a fixed $s$ with $\operatorname{Re} s \geq 0$ corresponding to the eigenvalue $\lambda=\lambda_{s}$ under consideration. The spaces $\mathcal{W}_{r}(v, s)$ are two-dimensional. We will use the basis elements in $\S 4.2$ of [Bruggeman 1994].

- For $\operatorname{Re} v \neq 0$, a basis of $\mathcal{W}_{r}(v, s)$ is formed by

$$
\begin{aligned}
& \omega_{r}(v, s ; z, \vartheta)=e^{2 \pi i v x} W_{r} \operatorname{Sign}(\operatorname{Re} v) / 2, s(4 \pi v \operatorname{Sign}(\operatorname{Re} v) y) e^{i r \vartheta}, \\
& \hat{\omega}_{r}(\nu, s ; z, \vartheta)=e^{2 \pi i v x} W_{-r} \operatorname{Sign}(\operatorname{Re} v) / 2, s(-4 \pi v \operatorname{Sign}(\operatorname{Re} v) y) e^{i r \vartheta} .
\end{aligned}
$$

Here $W_{\mu, s}(t)$ is the Whittaker function that decreases exponentially as $t \rightarrow \infty$. We use the branch of $W_{\kappa, s}(z)$ that is holomorphic for $-\pi / 2<\arg z<3 \pi / 2$. The asymptotic behavior as $y \rightarrow \infty$, by $\$ 4.2 .1$ in [Slater 1960], is:

$$
\begin{aligned}
& \omega_{r}(\nu, s ; z, \vartheta) \sim(4 \pi v \varepsilon y)^{r \varepsilon / 2} e^{2 \pi \nu(i x-\varepsilon y)+i r \vartheta}, \\
& \hat{\omega}_{r}(\nu, s ; z, \vartheta) \sim e^{-\pi i r \varepsilon / 2}(4 \pi \varepsilon v y)^{-r \varepsilon / 2} e^{2 \pi v(i x+\varepsilon y)+i r \vartheta},
\end{aligned}
$$

where $\varepsilon$ denotes $\operatorname{Sign}(\operatorname{Re} v)$. The subspace of $\mathcal{W}_{r}(v, s)$ generated by $\omega_{r}(v, s)$ is denoted by $\mathcal{W}_{r}^{0}(v, s)$.

- For $v=0$, a basis is given by $\left\{y^{1 / 2+s} e^{i r \vartheta}, y^{1 / 2-s} e^{i r \vartheta}\right\}$ if $s \neq 0$ and $\left\{y^{1 / 2} e^{i r \vartheta}\right.$, $\left.y^{1 / 2} \log y e^{i r \vartheta}\right\}$ if $s=0$.

The next proposition allows for Fourier expansions of functions with exponential growth. (See [Bruggeman 1994], §4.1-3 for a proof.)

Proposition 7.1. Let $k \in 2 \mathbb{Z}$, $\operatorname{Re} s \geq 0$. Suppose that the function $f \in C^{\infty}(\tilde{\Gamma} \backslash \tilde{G})$ satisfies $\omega f=\lambda_{s} f$ and $\boldsymbol{W} f=i k f$. Then it has at each cusp $\kappa$ an absolutely converging Fourier expansion

$$
f\left(\tilde{g}_{\kappa} g\right)=\sum_{n \in \mathbb{Z}} F_{\kappa, n} f(g),
$$

with $F_{\kappa, n} f \in \mathcal{W}_{k}(n, s)$. Moreover, $f \in \tilde{E}_{k}\left(\tilde{\Gamma}, \lambda_{s}\right)$ if and only if there exists $N>0$ such that all Fourier terms $F_{\kappa, n} f$ with $|n| \geq N$ are in $\mathcal{W}_{k}^{0}(n, s)$ for all cusps $\kappa$.

7B. Higher-order Fourier terms. The higher-order invariants of $\mathscr{V}_{k}(n, s)$ that we will define now are the higher-order analogues of the classical Fourier terms.

Definition 7.2. Let $k \in 2 \mathbb{Z}, n \in \mathbb{Z}$, and $s \in \mathbb{C}$. By $\mathscr{V}_{k}(n, s)$ we denote the space of functions $f$ on $\tilde{G}$ that satisfy $\omega f=\lambda_{s} f$, have generalized weight $k$, and satisfy $\left(\partial_{x}-2 \pi i n\right)^{m} f=0$ for some $m \in \mathbb{N}$ (which may depend on $f$ ). 
For $n \neq 0$, we denote by $\mathscr{V}_{k}^{0}(n, s)$ the subspace of $f \in \mathscr{V}_{k}(n, s)$ satisfying $f(z, \vartheta)=\mathrm{O}\left(y^{a} e^{-2 \pi|n| y}\right)$ as $y \rightarrow \infty$ for some $a \in \mathbb{R}$.

The free Abelian group $\tilde{\Delta}$ generated by $\tau=n(1)$ and $\zeta=k(\pi)$ acts on these spaces by left translation.

Proposition 7.3. Let $k, n, s$ be as above. The $\tilde{\Delta}$-modules $\mathscr{V}_{k}(n, s)$ and $\mathscr{V}_{k}^{0}(n, s)$ are maximally perturbable.

For each $q \in \mathbb{N}$, the elements $f \in \mathscr{V}_{k}(n, s)^{\tilde{\Delta}, q}$ satisfy for each $\delta>0$

$$
f(z, \vartheta) \ll_{\delta} e^{(2 \pi|n|+\delta) y}, \quad(y \rightarrow \infty)
$$

uniformly for $x$ and $\vartheta$ in compact sets. If $n \neq 0$, then for each $q \in \mathbb{N}$, the elements $f \in \mathscr{V}_{k}^{0}(n, s)^{\tilde{\Delta}, q}$ satisfy for each $\delta>0$

$$
f(z, \vartheta) \ll_{\delta} e^{(\delta-2 \pi|n|) y}, \quad(y \rightarrow \infty)
$$

uniformly for $x$ and $\vartheta$ in compact sets.

Proof. To prove that $\mathscr{V}_{k}(n, s)$ is maximally perturbable, we start with a characterization of the space $\mathscr{V}_{k}(n, s)^{\tilde{\Delta}}$. We first note that $\mathcal{W}_{k}(n, s) \subset \mathscr{V}_{k}(n, s)^{\tilde{\Delta}}$. Conversely, if $f \in \mathscr{V}_{k}(n, s)^{\tilde{\Delta}}$, then the reasoning in the proof of Theorem 6.4 shows that the weight of $f$ is strict, and also that $\partial_{x} f=2 \pi i n f$, and hence $f(z, \vartheta)=e^{2 \pi i n x} f(i y, \vartheta)$. So $f \in \mathcal{W}_{k}(n, s)$. If, for $n \neq 0$, the function $f$ is also exponentially decreasing, it has to be a multiple of $\omega_{k}(n, s)$. Therefore, $\mathscr{V}_{k}^{0}(n, s)^{\tilde{\Delta}}=\mathscr{W}_{k}^{0}(n, s)$.

Let $f$ be an arbitrary element of $\mathscr{W}_{k}(n, s)$. Since each of the basis elements of $\mathcal{W}_{k}(n, s)$ is a specialization of a holomorphic family of elements of $\mathcal{W}_{r}(v, s)$, there is a holomorphic family of $h(r, v) \in \mathcal{W}_{r}(v, s)$ such that $h(k, n)=f$. We have $h(r, v ; n(\xi) k(\ell \pi)(z, \vartheta))=e^{2 \pi i v \xi+\pi i r \ell} h(r, v ; z, \vartheta)$ for $\xi \in \mathbb{R}$ and $\ell \in \mathbb{Z}$.

Next, consider the polynomials $Q_{q} \in \mathbb{Q}[X]$ of degree $q$, defined by

$$
\begin{aligned}
Q_{0} & =1, \\
Q_{q+1}(X+1)-Q_{q+1}(X) & =Q_{q}(X), \\
Q_{q}(0) & =0, \quad \text { for } q \geq 1 .
\end{aligned}
$$

Then for each $\boldsymbol{m}=\left(m_{1}, m_{2}\right), m_{j} \geq 0$, set

$$
h_{k}^{m}(n, s)=\left.Q_{m_{1}}\left(\frac{1}{\pi i} \partial_{r}\right) Q_{m_{2}}\left(\frac{1}{2 \pi i} \partial_{v}\right) h(r, v)\right|_{\nu=n, r=k} .
$$


Applying the differential operator $(1 / 2 \pi i) \partial_{v}^{a}$ on $h(r, v) \mid(\tau-1)=\left(e^{2 \pi i v}-1\right) h(r, v)$ we obtain

$$
\begin{aligned}
Q_{m_{2}}\left(\frac{1}{2 \pi i} \partial_{\nu}\right) h(r, v) \mid(\tau-1) & =\left(Q_{m_{2}}\left(\frac{1}{2 \pi i} \partial_{\nu}+1\right)-Q_{m_{2}}\left(\frac{1}{2 \pi i} \partial_{\nu}\right)\right) h(r, v) \\
& =Q_{m_{2}-1}\left(\frac{1}{2 \pi i} \partial_{\nu}\right) h(r, v) .
\end{aligned}
$$

Since $\tau, \zeta$ commute, this implies $h_{k}^{\boldsymbol{m}}(n, s) \mid(\tau-1)=h_{k}^{\left(m_{1}, m_{2}-1\right)}(n, s)$. Likewise, we obtain the transformation law $h_{k}^{\boldsymbol{m}}(n, s) \mid(\zeta-1)=h_{k}^{\left(m_{1}-1, m_{2}\right)}(n, s)$. Therefore, for $l_{1}+l_{2}=m_{1}+m_{2}\left(l_{1}, l_{2} \geq 0\right)$,

$$
h_{k}^{\left(m_{1}, m_{2}\right)}(n, s) \mid(\zeta-1)^{l_{1}}(\tau-1)^{l_{2}}=\delta_{m_{1}, l_{1}} \delta_{m_{2}, l_{2}} f,
$$

thus obtaining the maximal perturbability of $\mathscr{V}_{k}(n, s)$. For convenience, we shall call perturbations satisfying the transformation law (7-13) perturbations of type $\boldsymbol{m}$.

Based on $\mathscr{V}_{k}^{0}(n, s)^{\tilde{\Delta}}=\mathcal{W}_{k}^{0}(n, s)$, we deduce in an analogous way the maximal perturbability of $\mathscr{V}_{k}^{0}(n, s)$.

To prove (7-8) and (7-9), we first note that the maximal perturbability we have just shown implies that the functions $h^{\boldsymbol{m}}$ constructed from elements $f$ ranging over a basis of $\mathcal{W}_{k}(n, s)\left(\right.$ resp. $\left.\mathcal{W}_{k}^{0}(n, s)\right)$ induce a basis of the quotients $\mathscr{V}^{\tilde{\Delta}, q+1} / \mathscr{V}^{\tilde{\Delta}, q}$. Therefore, it suffices to show (7-8) and (7-9) for $h^{m}$ only. In the case $n \neq 0$, the family $h$ may be taken to be $\omega_{r}(v, s)$ or $\hat{\omega}_{r}(v, s)$ in (7-4). For these functions, the question reduces to the asymptotic behavior of $\partial_{t}^{j} \partial_{\kappa}^{l} W_{\kappa, s}(t)$, since the factors $e^{2 \pi i v x}$ and $e^{i r \vartheta}$ produce polynomials in $x$ and $\vartheta$, which yield constants when they vary through compact sets. The differentiation of $4 \pi \operatorname{Sign}(\operatorname{Re} v) v y$ yields only a power of $y$, which can be absorbed by the factor $e^{\delta y}$.

Differentiation of $W_{\kappa, s}(t)$ with respect to $t$ does not change the exponential part of the asymptotic behavior, since derivatives of $W_{\kappa, s}(t)$ are linear combinations of $W_{\kappa, s}(t)$ and $W_{\kappa+1, s}(t)$ with powers of $t$ in the factors [Slater 1960, (2.4.24)]. So we have to look only at differentiation with respect to $\kappa$. Then the bounds are a consequence of the integral representation

$$
W_{\kappa, s}(t)=\frac{-1}{2 \pi i} \Gamma\left(\kappa+\frac{1}{2}-s\right) e^{-t / 2} t^{\kappa} \int_{(0+)}^{\infty} e^{-x}(-x)^{s-\kappa-1 / 2}\left(1+\frac{x}{t}\right)^{s+\kappa-1 / 2} d x
$$

for $t \in \mathbb{R}$ with $t>0, \kappa-\frac{1}{2}-s \neq-1,-2, \ldots$ (see (3.5.18) in [Slater 1960]). Here the contour comes from $\infty$ along a line slightly above the positive real axis, encircles 0 with radius $\delta<1$, and then goes back to $\infty$ on a line slightly below the positive real axis. If $\kappa-\frac{1}{2}-s=-1,-2, \ldots$, we use the representation

$$
W_{\kappa, s}(t)=\frac{e^{-(1 / 2) t} t^{\kappa} e^{i \varphi(s-\kappa+1 / 2)}}{\Gamma\left(s+\frac{1}{2}-\kappa\right)} \int_{0}^{\infty} e^{-e^{i \varphi} u} u^{s-\kappa-1 / 2}\left(1+e^{i \varphi} u / t\right)^{s+\kappa-1 / 2} d u,
$$


for some $0<\varphi<\pi / 2$.

All these estimates, taken together, prove (7-8) and (7-9) (when $n \neq 0$ ). They further show that the derivatives of a family with exponential decay have exponential decay, and thus $\mathscr{V}_{k}^{0}(n, s)$ is also maximally perturbable.

If $n=0$, we argue directly that we can find functions $h_{k}^{m}(0, s)$ in $\mathscr{V}_{k}(0, s)$ of the form $p_{\boldsymbol{m}}(x, y, \vartheta) y^{1 / 2 \pm 2} e^{i k \vartheta}$, where $p_{\boldsymbol{m}}$ is a polynomial in three variables with degree $m_{1}$ in $\vartheta$ and degree $m_{2}$ in $x$. If the coefficient of $\vartheta^{m_{1}} x^{m_{2}}$ in this polynomial does not depend on $y$, this leads to a perturbation of $y^{1 / 2 \pm s} e^{i k \vartheta}$ of type $\boldsymbol{m}$. Such functions satisfy the required estimates, with a polynomial factor $y^{A}$ instead of $e^{\delta y}$. The remaining task is to check that they can be chosen to satisfy

$$
\left(\omega-\frac{1}{4}+s^{2}\right) h_{k}^{m}(0, s)=0 .
$$

We do this by induction in the degrees in $\vartheta$ and $x$. We check that

$$
\begin{aligned}
& \left(\omega-\frac{1}{4}+s^{2}\right) x^{m_{2}} y^{1 / 2 \pm s+a} \vartheta^{m_{1}} e^{i k \vartheta} \\
& \quad=-a(a \pm 2 s) x^{m_{2}} y^{1 / 2 \pm s+a} \vartheta^{m_{1}} e^{i k \vartheta}+\text { terms of lower degree in } x \text { or } \vartheta .
\end{aligned}
$$

With $a=0$, this gives the top coefficient of $p_{m}$. Moreover, the terms of lower degree all are multiples of $x^{\tilde{m}_{2}} y^{1 / 2 \pm s+a} \vartheta^{\tilde{m}_{1}} e^{i k \vartheta}$ with $\tilde{m}_{j} \leq m_{j}, \tilde{m}_{1}<m_{1}$, or $\tilde{m}_{2}<m_{2}$, and $a \in \mathbb{Z}_{\geq 0}$. Successively we can determine the lower-degree terms, and arrange for $h_{k}^{m}(0, s)$ to be an eigenfunction of $\omega$ with eigenvalue $\frac{1}{4}-s^{2}$.

This takes care of the case $n=0$, except if $s=0$. It that case we also have to perform a computation involving $y^{1 / 2+a} \log y$, which we leave to the reader.

Holomorphic Fourier terms on $\tilde{G}$ are multiples of

$$
\eta_{r}(\nu ; z, \vartheta)=y^{r / 2} e^{2 \pi i v z} e^{i r \vartheta} .
$$

Thus we have the spectral parameter $s= \pm(r-1) / 2$. For real values of $v$ and $r$, we have

$$
\eta_{r}(v)= \begin{cases}(4 \pi v)^{-r / 2} \omega_{r}\left(v, \pm \frac{r-1}{2}\right) & \text { if } v>0, \\ \mu_{r}\left(0, \frac{r-1}{2}\right) & \text { if } v=0, \\ e^{-\pi i r}(4 \pi|v|)^{-r / 2} \hat{\omega}_{r}\left(v, \pm \frac{r-1}{2}\right) & \text { if } v<0,\end{cases}
$$

with notations as in (7-4) and (6-11). The functions

$$
\eta_{k}^{m}(n ; z, \vartheta)=Q_{m_{1}}\left(\frac{2 i \vartheta+\log y}{2 \pi i}\right) Q_{m_{2}}(z) \eta_{k}(n ; z, \vartheta)
$$

satisfy

$$
\mathrm{m}_{m_{1}+m_{2}} \eta_{k}^{m}:(\zeta-1)^{l_{1}}(\tau-1)^{l_{2}} \mapsto \delta_{m_{1}, l_{1}} \delta_{m_{2}, l_{2}} \eta_{k}(n)
$$


for $l_{1}+l_{2}=m_{1}+m_{2}$, and as $y \rightarrow \infty$, their growth is of order $\mathrm{O}\left(e^{(\delta-2 \pi n) y}\right)$. For the commutative group $\tilde{\Delta}$ and for a fixed $\boldsymbol{m}$, they yield a basis of the space of forms of order $m_{1}+m_{2}+1$ modulo lower-order forms.

As an example, we note that the Fourier expansion (6-1) can be written in the following way:

$$
L(z, \vartheta)=\pi i \eta_{0}^{(1,0)}(0 ; z, \vartheta)+\frac{\pi i}{6} \eta_{0}^{(0,1)}(0 ; z, \vartheta)-2 \sum_{n \geq 1} \sigma_{-1}(n) \eta_{0}^{(0,0)}(n ; z, \vartheta) .
$$

\section{Proofs of Theorems 6.5 and 6.8}

The method of the proof is highly inductive. At each step, we use the maximal perturbability of other spaces, which has been proved in a previous step. The starting point for this process is the $\operatorname{space} \operatorname{Map}(\tilde{\Gamma}, \mathbb{C})$, whose maximal perturbability is proved based on general algebraic principles in Proposition 8.1. This implies directly the maximal perturbability of the $\tilde{\Gamma}$-module $\operatorname{Map}(\mathfrak{H} \times \mathbb{R}, \mathbb{C})$. We proceed by imposing increasingly stringent regularity conditions on the functions $\mathfrak{H} \times \mathbb{R} \rightarrow \mathbb{C}$. We consider $C^{\infty}(\mathfrak{H} \times \mathbb{R})=C^{\infty}(\tilde{G})$, the subspace $C_{k}^{\infty}(\tilde{G})$ of functions in $C^{\infty}(\tilde{G})$ with generalized weight $k$, and the subspace $\mathscr{C}_{k}$ of $C_{k}^{\infty}(\tilde{G})$ of functions that have compact support modulo $\tilde{\Gamma}$. In Section 7 , we considered higher-order invariant functions for the group $\tilde{\Delta}$ generated by $n(1)$ and $k(\pi)$. These functions are related to the Fourier expansions of Maass forms. After proving that some more auxiliary subspaces of $C_{k}^{\infty}(\mathfrak{H} \times \mathbb{R})$ are maximally perturbable, we finally prove in Section $8 \mathrm{E}$ the maximal perturbability of $\tilde{\mathscr{E}}_{k}(\tilde{\Gamma}, \lambda)$ and $\mathscr{H}_{k}(\tilde{\Gamma})$.

\section{A. Higher-order invariants in maps on $\tilde{\boldsymbol{\Gamma}}$.}

Proposition 8.1. If $\tilde{\Gamma}$ is a discrete cofinite subgroup of $\tilde{G}$ with cusps, then the $\tilde{\Gamma}$-module $\operatorname{Map}(\tilde{\Gamma}, \mathbb{C})$ (with the action by left translation) is maximally perturbable. Proof. We first define $\boldsymbol{g}_{\boldsymbol{i}}$ on the free subgroup $\tilde{\Gamma}_{0}$ of $\tilde{\Gamma}$ generated by $\alpha_{1}, \ldots, \alpha_{t(\Gamma)-1}$ for $\boldsymbol{i} \in\{1, \ldots, t(\Gamma)-1\}^{q}$ by the relations

$$
\begin{aligned}
\boldsymbol{g}_{()} & =1, & & \\
\boldsymbol{g}_{(j, \boldsymbol{i})} \mid\left(\alpha_{j}-1\right) & =\boldsymbol{g}_{\boldsymbol{i}}, & & \\
\boldsymbol{g}_{\boldsymbol{i}} \mid\left(\alpha_{j}-1\right) & =0, & & \text { if } \boldsymbol{i}(1) \neq j, \\
\boldsymbol{g}_{\boldsymbol{i}}(1) & =0, & & \text { if }|\boldsymbol{i}| \geq 1 .
\end{aligned}
$$

By $|\boldsymbol{i}|$ we denote the length of the tuple $\boldsymbol{i}$.

Let $\varphi_{0}: \tilde{\Gamma} \rightarrow \tilde{\Gamma}_{0}$ be the surjective group homomorphism given by $\varphi_{0}\left(\alpha_{j}\right)=\alpha_{j}$ for $1 \leq j \leq t(\Gamma)-1, \varphi_{0}(\zeta)=1$, and $\varphi_{0}\left(\varepsilon_{j}\right)=1$ for $1 \leq j \leq n_{\text {ell }}$. For $1 \leq j \leq t(\Gamma)$, we define $\psi_{j} \in \operatorname{hom}(\tilde{\Gamma}, \mathbb{C})$ such that $\psi_{j}\left(\alpha_{j^{\prime}}\right)=\delta_{j, j^{\prime}}$. This determines $\psi_{j}$ completely, because values on elliptic generators are given by $\psi_{j}\left(\varepsilon_{j}\right)=\left(1 / v_{j}\right) \psi_{j}(\zeta)$. For 
$\boldsymbol{i}=\left(\boldsymbol{i}^{\prime}, t(\Gamma), \ldots, t(\Gamma)\right)$, where there are $m$ coordinates $t(\Gamma)$ at the end and where $\boldsymbol{i}^{\prime} \in\{1, \ldots, t(\Gamma)-1\}^{q-m}$, we put

$$
\boldsymbol{f}_{\boldsymbol{i}}(\gamma)=\boldsymbol{g}_{\boldsymbol{i}^{\prime}}\left(\varphi_{0}(\gamma)\right) Q_{m}\left(\psi_{t(\Gamma)}(\gamma)\right),
$$

where $Q_{n}$ are the polynomials defined in (7-10). Now we can check the following properties of $f_{i}$ :

$$
\begin{aligned}
& \boldsymbol{f}_{()}=1 \quad \text { (empty tuple, } q=0 \text { ); } \\
& \boldsymbol{f}_{\boldsymbol{i}}(1)=0 \quad \text { if }|\boldsymbol{i}| \geq 1 \text {; } \\
& f_{i} \mid(\zeta-1)= \begin{cases}f_{i^{\prime}} & \text { if } \boldsymbol{i}=\left(\boldsymbol{i}^{\prime}, t(\Gamma)\right), \\
0 & \text { if } \boldsymbol{i} \text { does not end with a } t(\Gamma) ;\end{cases} \\
& f_{i} \mid\left(\alpha_{j}-1\right)= \begin{cases}f_{i^{\prime}} & \text { if } i=\left(j, i^{\prime}\right) \text { with } j<t(\Gamma), \\
0 & \text { if } j<t(\Gamma), j \neq i(1) .\end{cases}
\end{aligned}
$$

Using this, we can see that

$$
\left(\mathrm{m}_{q} \boldsymbol{f}_{\boldsymbol{i}}\right)(\boldsymbol{b}(\boldsymbol{j}))=\delta_{\boldsymbol{i}, \boldsymbol{j}}
$$

Now, the choice of the basis $\boldsymbol{b}(\boldsymbol{i})$ in (5-7) for $\tilde{\Gamma}$ - $q$-tuples $\boldsymbol{i}$ shows that to prove that $\operatorname{Map}(\tilde{\Gamma}, \mathbb{C})$ is maximally perturbable, it suffices to prove that for each $\boldsymbol{i}$ and for each function $f$ on $\tilde{\Gamma} \backslash \tilde{G}$, a function $h_{i} \in \operatorname{Map}(\tilde{G}, \mathbb{C})$ such that, for all $\tilde{\Gamma}$ - $q$-tuples $j$,

$$
h_{i} \mid\left(\alpha_{j(1)}-1\right) \ldots\left(\alpha_{j(q)}-1\right)=\delta_{i, j} \cdot f .
$$

To construct such functions, we choose a strict fundamental domain $\mathfrak{F}_{\tilde{\Gamma}} \subset \tilde{G}$ for $\tilde{\Gamma} \backslash \tilde{G}$, that is, a set meeting each $\tilde{\Gamma}$-orbit exactly once. A choice for the sought function $h_{i}$ is then

$$
h_{i}(\gamma g)=f_{i}(\gamma) f(g), \quad \gamma \in \Gamma, g \in \mathfrak{F}_{\tilde{\Gamma}}
$$

or

$$
h_{i}(g)=\sum_{\gamma \in \tilde{\Gamma}} f_{i}(\gamma) f(g) \psi\left(\gamma^{-1} g\right),
$$

where $\psi$ is the characteristic function of $\mathfrak{F}_{\tilde{\Gamma}}$.

8B. Higher-order invariants in smooth functions on $\tilde{\boldsymbol{G}}$. We will use essentially the same construction as in the last section to prove:

Proposition 8.2. The $\tilde{\Gamma}$-module $C^{\infty}(\tilde{G})$ is maximally perturbable.

Proof. In order to show that $C^{\infty}(\tilde{G})$ is a maximally perturbable $\tilde{\Gamma}$-module, we need to have (8-8) with $h_{i} \in C^{\infty}(\tilde{G})$ for each $f \in C^{\infty}(\tilde{\Gamma} \backslash \tilde{G})$. We consider functions $\psi \in C^{\infty}(\mathfrak{H} \times \mathbb{R})$ such that $\sum_{\gamma \in \tilde{\Gamma}} \psi\left(\gamma^{-1}(z, \vartheta)\right)=1$ for all $(z, \vartheta) \in \mathfrak{H} \times \mathbb{R}$ as a locally finite sum. If we define (8-10) with such a function $\psi$ and $f \in C^{\infty}(\tilde{\Gamma} \backslash \tilde{G})$, then the sum is locally finite, and the $h_{i}$ are smooth. 
8C. Higher-order invariants and generalized weight. Set

$$
C_{k}^{\infty}(\tilde{G})=\left\{f \in C^{\infty}(\tilde{G}) \text {, of generalized weight } k\right\} .
$$

Proposition 8.3. Let $k \in 2 \mathbb{Z}$. Then the $\tilde{\Gamma}$-module $C_{k}^{\infty}(\tilde{G})$ is maximally perturbable. Proof. As with the previous proofs, our approach is to show that for every $\tilde{\Gamma}-q$-tuple $\boldsymbol{i}=\left(\boldsymbol{i}^{\prime}, t(\Gamma), \ldots, t(\Gamma)\right)$ with exactly $m$ occurrences of $t(\Gamma)$ at the end and for every $f \in C_{k}^{\infty}(\tilde{\Gamma} \backslash \tilde{G})$, there exists $h_{i} \in C_{k}^{\infty}(\tilde{G})$ satisfying Equation (8-8) for all $\tilde{\Gamma}$-q-tuples $\boldsymbol{j}$. We note that, by Theorem 6.4 , the $\tilde{\Gamma}$-invariance of $f$ implies that its weight $k$ is strict, that is, $f(g k(\vartheta))=f(g) e^{i k \vartheta}$.

We will define the function $h_{i}$ by an analogue of (8-10). We first define for each $g \in \tilde{G}$ the point $w(g)=\operatorname{pr}(g) i \in \mathfrak{H}$ and the real number $\Theta(g) \in \mathbb{R}$ such that $g=(w(g), \Theta(g)) \in \tilde{G}=\mathfrak{H} \times \mathbb{R}$. We also recall that $\Gamma=\tilde{\Gamma} / \tilde{Z}$. Since the group homomorphism $\phi_{0}$ defined in the proof of Proposition 8.1 is trivial on $\tilde{Z}=\langle\zeta\rangle$, it induces a homomorphism on $\Gamma$. Now we take $\psi(z, \vartheta)$ to be a bounded locally finite partition of unity $\psi_{0}$ on $\mathfrak{H}$. (Compare Lemma 1 in $\S 3$ of [Kra 1969].) So the function $(z, \vartheta) \mapsto \psi\left(\gamma^{-1}(z, \vartheta)\right)$ obtained by left translation depends only on the image of $\gamma \in \tilde{\Gamma}$ in $\Gamma \cong \tilde{\Gamma} / \tilde{Z}$. Let, as in the proof of Proposition 8.1, $\psi_{t(\Gamma)}$ be the function $\tilde{\Gamma} \rightarrow \mathbb{R}$ such that $\psi_{t(\Gamma)}\left(\alpha_{j^{\prime}}\right)=\delta_{t(\Gamma), j^{\prime}}$. For a given $\gamma \in \tilde{\Gamma}$, we have

$$
\psi_{t(\Gamma)}(\zeta \gamma)=\psi_{t(\Gamma)}(\gamma)+1
$$

and $\Theta\left((\zeta \gamma)^{-1} g\right)=\Theta\left(\gamma^{-1} g\right)-\pi$. So $\psi_{t(\Gamma)}(\gamma)+\Theta\left(\gamma^{-1} g\right) / \pi$ is well-defined on $\Gamma=\tilde{\Gamma} / \tilde{Z}$. We can therefore set

$$
h_{\boldsymbol{i}}(g)=\sum_{\gamma \in \Gamma} \boldsymbol{g}_{\boldsymbol{i}^{\prime}}\left(\varphi_{0}(\gamma)\right) Q_{m}\left(\psi_{t(\Gamma)}(\gamma)+\Theta\left(\gamma^{-1} g\right) / \pi\right) f(g) \psi\left(\gamma^{-1} g\right) .
$$

The support property of the partition of unity $\psi$ ensures convergence; it is even a locally finite sum with a bounded number of nonzero terms. All factors depend smoothly on $g$. So $h_{i} \in C^{\infty}(\tilde{G})$.

We consider $(\boldsymbol{W}-i k) h_{i}$. Since $\boldsymbol{W} \psi=0$, we need only consider

$$
\begin{aligned}
\left(\partial_{\vartheta}-i k\right) Q_{m}\left(\psi_{t(\Gamma)}(\gamma)+\Theta\left(\gamma^{-1} g k(\vartheta)\right) / \pi\right) f(g k(\vartheta)) & \\
= & Q_{m}\left(\psi_{t(\Gamma)}(\gamma)+\Theta\left(\gamma^{-1} g k(\vartheta)\right) / \pi\right)\left(\partial_{\vartheta}-i k\right) f(g k(\vartheta)) \\
& +f(g k(\vartheta)) \partial_{\vartheta} Q_{m}\left(\psi_{t(\Gamma)}(\gamma)+\Theta\left(\gamma^{-1} g\right) / \pi+\vartheta / \pi\right) \\
=0 & +\pi^{-1} Q_{m}^{\prime}\left(\psi_{t(\Gamma)}(\gamma)+\Theta\left(\gamma^{-1} g\right) / \pi+\vartheta / \pi\right) f(g k(\vartheta)) .
\end{aligned}
$$

Repeating this, we obtain

$$
\begin{aligned}
(\boldsymbol{W}-i k)^{m+1} Q_{m}\left(\psi_{t(\Gamma)}(\gamma)+\Theta\left(\gamma^{-1} g\right) / \pi\right) f(g) & \\
& =\pi^{-m-1} Q_{m}^{(m+1)}(\ldots) \ldots \\
& =0,
\end{aligned}
$$


since the degree of $Q_{m}$ is $m$. So $h_{i} \in C_{k}^{\infty}(\tilde{G})$.

Remark. As A. Deitmar has pointed out, the last two propositions should also follow from [Deitmar 2008]. We have opted for explicit methods of proof because they are necessary for later parts of the paper.

8D. Higher-order invariants with support conditions. We discuss the motivation for the introduction of the invariants we will be dealing with. If Definition 6.3 of the space $\tilde{\mathscr{E}}_{k}(\tilde{\Gamma}, \lambda)$ did not include a growth condition at the cusps, we could consider $\tilde{\mathscr{E}}_{k}(\tilde{\Gamma}, \lambda)$ as the kernel $\mathscr{K}$ in the exact sequence

$$
0 \longrightarrow \mathscr{K} \longrightarrow C_{k}^{\infty}(\tilde{G}) \stackrel{\omega-\lambda}{\longrightarrow} C_{k}^{\infty}(\tilde{G}) .
$$

With exponential growth, one might want to try to replace $C_{k}^{\infty}(\tilde{G})$ by its subspace $C_{l}^{\infty}(\tilde{\Gamma})^{\text {eg }}$ of functions with exponential growth at the cusps of $\tilde{\Gamma}$. This would lead to an exact sequence

$$
0 \longrightarrow \tilde{\mathscr{E}}_{k}(\tilde{\Gamma}, \lambda) \longrightarrow C_{k}^{\infty}(\tilde{\Gamma})^{\mathrm{eg}} \stackrel{\omega-\lambda}{\longrightarrow} C_{k}^{\infty}(\tilde{\Gamma})^{\mathrm{eg}},
$$

for which we might try to show that for each $q \in \mathbb{N}$,

$$
0 \longrightarrow \tilde{\mathscr{E}}_{k}(\tilde{\Gamma}, \lambda)^{\tilde{\Gamma}, q} \longrightarrow\left(C_{k}^{\infty}(\tilde{\Gamma})^{\mathrm{eg}}\right)^{\tilde{\Gamma}, q} \stackrel{\omega-\lambda}{\longrightarrow}\left(C_{k}^{\infty}(\tilde{\Gamma})^{\mathrm{eg}}\right)^{\tilde{\Gamma}, q}
$$

is exact. For this to be of use, it seems that we need surjectivity of the map

$$
\omega-\lambda:\left(C_{k}^{\infty}(\tilde{\Gamma})^{\mathrm{eg}}\right)^{\tilde{\Gamma}} \rightarrow\left(C_{k}^{\infty}(\tilde{\Gamma})^{\mathrm{eg}}\right)^{\tilde{\Gamma}},
$$

which we did not succeed in proving, and which may not hold. For this reason, we will instead work with other, better behaved subspaces of the spaces appearing in the exact sequence. We will therefore define subspaces $\mathscr{C}_{k}, \mathscr{D}_{k}(\lambda) \subset C_{k}^{\infty}(\tilde{G})$ and $\mathscr{E}_{k}^{\prime}(\lambda) \subset \mathscr{E}_{k}(\tilde{\Gamma}, \lambda)$, related by an exact sequence

$$
0 \longrightarrow \tilde{\mathscr{E}}_{k}^{\prime}(\lambda) \longrightarrow \mathscr{D}_{k}(\lambda) \stackrel{\omega-\lambda}{\longrightarrow} \mathscr{C}_{k} .
$$

8D1. The spaces $\mathscr{C}_{k}$. For each cusp $\kappa=\tilde{g}_{\kappa} \infty$ and each $a>0$, we call

$$
D_{\kappa}(a)=\tilde{g}_{\kappa}\{(z, \vartheta): \operatorname{Im} z \geq a, \vartheta \in \mathbb{R}\}
$$

a horocyclic set. There is a number $A_{\Gamma}$ such that for each $a \geq A_{\Gamma}$, the $D_{\kappa}(a)$ are disjoint for different cusps. The sets

$$
\tilde{G}_{a}=\left\{(z, \vartheta) \in \mathfrak{H} \times \mathbb{R}:(z, \vartheta) \notin D_{\kappa}(a) \text { for all } \kappa\right\}
$$

satisfy $\tilde{\Gamma} \tilde{G}_{a}=\tilde{G}_{a}$. This follows from the fact that the $g_{\kappa}$ have been chosen so that

$$
\gamma \tilde{\Gamma}_{\kappa} \tilde{g}_{\kappa}=\tilde{g}_{\gamma \kappa} \tilde{\Gamma}_{\infty},
$$

for all cusps $\kappa$ and for $\gamma \in \tilde{\Gamma}$. Here $\tilde{\Gamma}_{\kappa}:=\operatorname{pr}^{-1} \Gamma_{\kappa}=\{\gamma \in \tilde{\Gamma}: \gamma \kappa=\kappa\}$. 
Definition 8.4. Let $k \in 2 \mathbb{Z}$. The space $\mathscr{C}_{k}$ consists of the $f \in C_{k}^{\infty}(\tilde{G})$ supported in $\tilde{G}_{a}$ for some $a \geq A_{\Gamma}$. (The $a$ may depend on $f$ ).

So $\mathscr{C}_{k}$ consists of the smooth functions with generalized weight $k$ whose supports project to compact subsets of $\Gamma \backslash \mathfrak{H}$. Clearly, the space $\mathscr{C}_{k}$ is $\tilde{\Gamma}$-invariant. If we apply the construction of $h_{i}$ in the proof of Proposition 8.3 to functions $f \in \mathscr{C}_{k}^{\tilde{\Gamma}} \subset$ $C_{k}^{\infty}(\tilde{\Gamma} \backslash \tilde{G})$, then the support of each $h_{i}$ is contained in the same set $\tilde{G}_{a}$ that contains $\operatorname{Supp}(f)$. This implies:

Proposition 8.5. Let $k \in 2 \mathbb{Z}$. Then the $\tilde{\Gamma}$-module $\mathscr{C}_{k}$ is maximally perturbable.

8D2. The spaces $\mathscr{D}_{k}(\lambda)$. We will define $\mathscr{D}_{k}(\lambda)$ essentially as the space of functions that accept higher-order analogues of Fourier expansions at the cusps. To make this formal, we study spaces of functions defined for $y_{0}>0$ on regions of the form

$$
S\left(y_{0}\right)=\left\{(x+i y, \vartheta) \in \mathfrak{H} \times \mathbb{R}: y>y_{0}\right\} .
$$

Definition 8.6. Let $k \in 2 \mathbb{Z}, \lambda \in \mathbb{C}$, and $y_{0}>0$. We denote by $\mathscr{E}_{k}\left(y_{0}, \lambda\right)$ the space of those $f \in C^{\infty}\left(S\left(y_{0}\right)\right)$ that satisfy $\omega f=\lambda f$ and $(\boldsymbol{W}-i k)^{n} f=0$ for some $n \in \mathbb{N}$, and have at most exponential growth as $y \rightarrow \infty$, uniform for $x$ and $\vartheta$ in compact sets. We denote by $\mathscr{E}_{k}^{\mathrm{hol}}\left(y_{0}\right)$ the space of holomorphic functions on $S\left(y_{0}\right)$ with generalized weight $k$ and at most exponential growth as $y \rightarrow \infty$.

Proposition 8.7. Let $k \in 2 \mathbb{Z}, s \in \mathbb{C}$, and $y_{0}>0$. The spaces $\mathscr{E}_{k}\left(y_{0}, \lambda_{s}\right)$ and $\mathscr{E}_{k}^{\mathrm{hol}}\left(y_{0}\right)$ are maximally perturbable $\tilde{\Delta}$-modules.

Let $q \in \mathbb{N}$. Each $f \in \mathscr{E}_{k}\left(y_{0}, \lambda_{s}\right)^{\tilde{\Delta}, q}$ has an absolutely convergent expansion

$$
f(z, \vartheta)=\sum_{n \in \mathbb{Z}} f_{n}(z, \vartheta)
$$

on $S\left(y_{0}\right)$ with $f_{n} \in \mathscr{V}_{k}(n, s)^{\tilde{\Delta}, q}$ for all $n$, and $f_{n} \in \mathscr{V}_{k}^{0}(n, s)^{\tilde{\Delta}, q}$ for almost all $n$.

Each $f \in \mathscr{E}_{k}^{\text {hol }}\left(y_{0}\right)^{\tilde{\Delta}, q}$ has an absolutely convergent expansion on $S\left(y_{0}\right)$ of the form

$$
f(z, \vartheta)=\sum_{\boldsymbol{m}, m_{1}+m_{2}<q} \sum_{n} c_{\boldsymbol{m}}^{n} \eta_{k}^{\boldsymbol{m}}(n ; z, \vartheta),
$$

where the inner sum ranges from some, possible negative, integer to infinity.

Proof. We start with the holomorphic case. Let $f \in \mathscr{E}_{k}^{\text {hol }}\left(y_{0}\right)^{\tilde{\Delta}}$. Then the function $z \mapsto y^{-k / 2} f(z, 0)$ is holomorphic on $\left\{z \in \mathfrak{H}: y>y_{0}\right\}$ with period 1 . So it has an expansion of the form $\sum_{n} a_{n} e^{2 \pi i n z}$, finite to the left and converging absolutely on $y>y_{0}$. For each $y_{1}>y_{0}$, we have $a_{n}=\mathrm{O}\left(e^{2 \pi n y_{1}}\right)$ as $n \rightarrow \infty$.

Hence, $f(z, \vartheta)=\sum_{n} a_{n} \eta_{k}(n ; z, \vartheta)$ converges absolutely on $y>y_{0}$, and

$$
f^{\boldsymbol{m}}(z, \vartheta):=\sum_{n \geq-N} a_{n} \eta_{k}^{\boldsymbol{m}}(n ; z, \vartheta)
$$


converges absolutely on $S\left(y_{0}\right)$, and the convergence is uniform on any set $y \geq y_{1}$ with $y_{1}>y_{0}$, with $x$ and $\vartheta$ in compact sets. These functions satisfy $f^{m} \mid(\tau-1)=$ $f^{\left(m_{1}, m_{2}-1\right)}, f^{\boldsymbol{m}} \mid(\zeta-1)=f^{\left(m_{1}-1, m_{2}\right)}$, and $f^{(0,0)}=f$, since all $\eta_{k}^{\boldsymbol{m}}$ have this property. Thus $f^{\boldsymbol{m}}$, with $\boldsymbol{m}$ such that $m_{1}+m_{2}<q$, is a perturbation of type $\boldsymbol{m}$, and we deduce that $\mathscr{E}_{k}$ hol $\left(y_{0}\right)$ is maximally perturbable. An arbitrary element $h \in \mathscr{E}_{k}{ }^{\mathscr{h} o l}\left(y_{0}\right)^{\tilde{\Delta}, q}$ can be written as a finite linear combination of such $f^{m}$, which all have expansions of the type given in (8-21).

For $f \in \mathscr{E}_{k}\left(y_{0}, \lambda_{s}\right)^{\tilde{\Delta}}$ we proceed similarly. By Proposition 7.1 and the integrality of $k$, there is an absolutely convergent Fourier expansion

$$
f(z, \vartheta)=\sum_{n \in \mathbb{Z}} f_{n}(z, \vartheta)
$$

on $S\left(y_{0}\right)$ with $f_{n} \in \mathcal{W}_{k}(n, s)$. By the exponential growth, $f_{n} \in \mathcal{W}_{k}^{0}(n, s)$ for $|n|>N$, for some $N \in \mathbb{N}$.

For $|n|>N$, we have $f_{n}=a_{n} \omega_{k}(n, s)$, and from (7-5) we conclude that $a_{n}=$ $\mathrm{O}\left(e^{2 \pi|n| y_{1}}\right)$ as $|n| \rightarrow \infty$ for each $y_{1}>y_{0}$. So by (7-5), the series

$$
\sum_{n,|n|>N} a_{n} \omega_{k}^{\boldsymbol{m}}(n, s)
$$

converges absolutely on $S\left(y_{0}\right)$ and uniformly on each set $y \geq y_{1}$ with $y_{1}>y_{0}$, and gives an exponentially decreasing function as $y \rightarrow \infty$. It is a $\lambda_{s}$-eigenfunction of $\omega$, since the decay allows differentiation inside the sum. To produce a perturbation $f^{\boldsymbol{m}}$ of $f$, we pick $f_{n}^{\boldsymbol{m}} \in \mathscr{V}_{k}(n, s)^{\tilde{\Delta}, m_{1}+m_{2}+1}$ such that $f_{n}^{\boldsymbol{m}} \mid(\tau-1)=f_{n}^{\left(m_{1}, m_{2}-1\right)}$, $f_{n}^{\boldsymbol{m}} \mid(\zeta-1)=f_{n}^{\left(m_{1}-1, m_{2}\right)}$, and $f_{n}^{(0,0)}=f_{n}$ for the finitely many $n$ with $|n| \leq N$. The estimate (7-8) shows that the growth of these terms is at most of the order $\mathrm{O}\left(e^{(2 \pi N+\delta) y}\right)$ as $y \rightarrow \infty$ for each $\delta>0$. Thus we get (nonuniquely) a perturbation of type $\boldsymbol{m}$ in $\mathscr{E}_{k}\left(y_{0}, \lambda_{s}\right)$ :

$$
f^{\boldsymbol{m}}=\sum_{|n| \leq N} f_{n}^{\boldsymbol{m}}+\sum_{|n|>N} a_{n} \omega_{k}^{\boldsymbol{m}}(n, s) .
$$

Thus we get (8-20) and the maximal perturbability of $\mathscr{E}_{k}\left(y_{0}, \lambda_{s}\right)$.

We are now ready to define $\mathscr{D}_{k}(\lambda)$ and $\mathscr{D}_{k}^{\text {hol }}$.

Definition 8.8. Let $k \in 2 \mathbb{Z}$ and $\lambda \in \mathbb{C}$. We define $\mathscr{D}_{k}(\lambda)$ as the space of functions $f \in C_{k}^{\infty}(\tilde{G})$ (hence with generalized weight $k$ ) for which there exist $b \geq A_{\Gamma}, a \in \mathbb{R}$, and $q \in \mathbb{N}$ such that for each cusp $\kappa$ of $\tilde{\Gamma}$, the function $(z, \vartheta) \mapsto f\left(\tilde{g}_{\kappa}(z, \vartheta)\right)$ is an element of $\mathscr{E}_{k}(b, \lambda)^{\tilde{\Delta}, q}$ and satisfies a bound $\mathrm{O}\left(e^{a y}\right)$ as $y \rightarrow \infty$.

We define $\mathscr{D}_{k}^{\text {hol }}$ similarly, with $(z, \vartheta) \mapsto f\left(\tilde{g}_{\kappa}(z, \vartheta)\right)$ in $\mathscr{E}_{k}^{\text {hol }}(b)^{\tilde{\Delta}, q}$, with bound $\mathrm{O}\left(e^{a y}\right)$.

Remark 8.9. The numbers $a, b$, and $q$ may depend on the function $f$. 
Remark 8.10. Definition 8.6 of $\mathscr{E}_{k}(b, \lambda)$ implies that elements of $\mathscr{D}_{k}(\lambda)$ are $\lambda$ eigenfunctions of $\omega$ on the set $\bigsqcup_{\kappa} D_{\kappa}(b)$. Similarly, elements of $\mathscr{D}_{k}^{\text {hol }}$ are holomorphic functions on $\bigsqcup_{\kappa} D_{\kappa}(b)$. In both cases, we have exponential growth at each cusp. The definition requires that the order of this exponential growth stay bounded when we vary the cusp.

The space $\mathscr{C}_{k}$ is contained in $\mathscr{D}_{k}(\lambda)$ and in $\mathscr{D}_{k}^{\text {hol }}$. Indeed, for given $f \in \mathscr{C}_{k}$, we can take $b$ large so that $\bigsqcup_{\kappa} D_{\kappa}(b)$ is outside the support of $f$. Elements $f$ of $\tilde{\mathscr{C}}_{k}(\tilde{\Gamma}, \lambda)^{\tilde{\Gamma}}$ restricted to $D_{\kappa}(b)$ induce elements $(z, \vartheta) \mapsto f\left(\tilde{g}_{\kappa}(z, \vartheta)\right)$ in $\mathscr{E}_{k}(b, \lambda)^{\tilde{\Delta}}$ for each cusp $\kappa$, and similarly in the holomorphic case. Hence

$$
\tilde{\mathscr{E}}_{k}(\tilde{\Gamma}, \lambda)^{\tilde{\Gamma}} \subset \mathscr{D}_{k}(\lambda)^{\tilde{\Gamma}}, \quad \mathscr{H}_{k}(\tilde{\Gamma})^{\tilde{\Gamma}} \subset\left(\mathscr{D}_{k}^{\mathrm{hol}}\right)^{\tilde{\Gamma}} .
$$

Maximal perturbability of $\mathscr{D}_{k}(\lambda)$ and $\mathscr{D}_{k}^{\text {hol }}$. We need a technical lemma in order to relate $\tilde{\Delta}$-invariants to $\tilde{\Gamma}$-invariants.

We first note that if $\infty$ is a cusp of $\tilde{\Gamma}$ and if $\tilde{g}_{\infty}=1$, then $\tilde{\Delta}=\tilde{\Gamma}_{\infty}$. In general, the group $\tilde{\Gamma}_{\kappa}$ can be conjugated to $\tilde{g}_{\kappa}^{-1} \tilde{\Gamma}_{\kappa} \tilde{g}_{\kappa}=\tilde{\Delta}$ in $\tilde{g}_{\kappa}^{-1} \tilde{\Gamma} \tilde{g}_{\kappa}$. So we can assume here that $\tilde{\Delta} \subset \tilde{\Gamma}$.

The abelian group $\tilde{\Delta}$ is free on the generators $\tau=n(1)$ and $\zeta=k(\pi)$. The $\operatorname{dimension}$ of $\operatorname{Map}(\tilde{\Delta}, \mathbb{C})^{\tilde{\Delta}, q+1}$ is $(q+1)(q+2) / 2$, with an explicit basis described as follows. Define a sequence of maps on $\tilde{\Delta}$ by setting

$$
\begin{aligned}
& \varphi^{(l, m)} \mid(\zeta-1)=\varphi^{(l-1, m)}, \\
& \varphi^{(l, m)} \mid(\tau-1)=\varphi^{(l, m-1)}, \\
& \varphi^{(0,0)}=1, \quad \varphi^{(l, m)}=0, \quad \text { for } l \text { or } m \text { negative },
\end{aligned}
$$

and

$$
\varphi^{(l, m)}(1)=0, \quad \text { for } l, m \geq 0, l+m>0 .
$$

Then

$$
\left(\mathrm{m}_{q} \varphi^{(l, m)}\right)\left(\left(\zeta^{r}-1\right)\left(\tau^{s}-1\right)\right)=\delta_{l, r} \delta_{m, s}
$$

for $l+m=r+s=q$, and therefore the $\varphi^{(l, m)}$ with $l, m \geq 0, l+m \leq q$ is a basis of $\operatorname{Map}(\tilde{\Delta}, \mathbb{C})^{\tilde{\Delta}, q+1}$.

Let $R$ be a system of representatives of $\tilde{\Gamma} / \tilde{\Delta}$; so $R \subset \tilde{\Gamma}$. Consider the system $\left\{\boldsymbol{f}_{\boldsymbol{j}}\right\}_{|\boldsymbol{j}|=q} \subset \operatorname{Map}(\tilde{\Gamma}, \mathbb{C})^{\tilde{\Gamma}, q+1}$ in the proof of Proposition 8.1. If $|\boldsymbol{j}|=q$, then for every $\gamma \in \tilde{\Gamma}, \delta \mapsto \boldsymbol{f}_{j}(\gamma \delta)$ is a function on $\tilde{\Delta}$ of order at most $q+1$. Hence, there are functions $a_{l, m}^{j}$ on $R$ such that for all $\rho \in R$ and $\delta \in \tilde{\Delta}$,

$$
\boldsymbol{f}_{\boldsymbol{j}}(\rho \delta)=\sum_{\substack{l, m \geq 0 \\ l+m \leq q}} a_{l, m}^{\boldsymbol{j}}(\rho) \varphi^{(l, m)}(\delta) .
$$


Lemma 8.11. Let $a_{l, m}^{j}$ be as in (8-24), and suppose that we have functions $\psi^{(l, m)} \in$ $\operatorname{Map}(\tilde{\Delta}, \mathbb{C})$ satisfying

$$
\begin{array}{rlrl}
\psi^{(0,0)} & =0, & \\
\psi^{(l, m)} \mid(\tau-1) & =\psi^{(l-1, m)} & & \text { for } l \geq 1, \\
\psi^{(l, m)} \mid(\zeta-1) & =\psi^{(l, m-1)} & & \text { for } m \geq 1 .
\end{array}
$$

Then

$$
f(\rho \delta)=\sum_{\substack{l, m \geq 0 \\ l+m \leq q}} a_{l, m}^{j}(\rho) \psi^{(l, m)}(\delta), \quad(\rho \in R, \delta \in \tilde{\Delta})
$$

defines an element of $\operatorname{Map}(\tilde{\Gamma}, \mathbb{C})^{\tilde{\Gamma}, q}$.

Proof. We proceed by induction in $q=|\boldsymbol{j}|$. If $q=0$, then $m=n=0$, so $f(\rho \delta)=a_{0,0}^{j}(\rho) \cdot \psi^{(0,0)}=0 \in \operatorname{Map}(\tilde{\Gamma}, \mathbb{C})^{\tilde{\Gamma}, 0}=\{0\}$.

It is clear that (8-26) gives a well-defined map on $\tilde{\Gamma}$. It suffices to prove that, for any generator $\alpha_{j}$ of $\tilde{\Gamma}, f \mid\left(\alpha_{j}-1\right) \in \operatorname{Map}(\tilde{\Gamma}, \mathbb{C})^{q-1}$. Suppose first that $\boldsymbol{j}=\left(j, \boldsymbol{j}^{\prime}\right)$. For each $\rho \in R$, there are unique $\rho_{1} \in R$ and $\delta_{1} \in \tilde{\Delta}$ such that $\alpha_{j} \rho=\rho_{1} \delta_{1}$. From (8-24), it follows that

$$
\begin{aligned}
f_{j}\left|\left(\alpha_{j(1)}-1\right)(\rho \delta)=\sum_{\substack{l, m \geq 0 \\
l+m \leq q}} a_{l, m}^{j}\left(\rho_{1}\right) \varphi^{(l, m)}\right|\left(\delta_{1}-1\right)(\delta) \\
+\sum_{\substack{l, m \geq 0 \\
l+m \leq q}}\left(a_{l, m}^{j}\left(\rho_{1}\right)-a_{l, m}^{j}(\rho)\right) \varphi^{(l, m)}(\delta) .
\end{aligned}
$$

By (8-6), the left-hand side equals

$$
\sum_{\substack{l, m \geq 0 \\ l+m \leq q-1}} a_{l, m}^{j^{\prime}}(\rho) \phi^{(l, m)}(\delta) .
$$

The function $\varphi^{(l, m)} \mid\left(\delta_{1}-1\right)$ is a linear combination, depending on $\rho$, of $\varphi^{(a, b)}$ with $0 \leq a \leq l, 0 \leq b \leq m$, and $a+b \leq q-1$. Thus we get an expression for the $a_{l, m}^{j^{\prime}}(\rho)$ in terms of the $a_{l, m}^{j}(\rho)$. The form of this expression depends on the relations (8-23), but not on the specific value of the constant basis element $\varphi^{(0,0)}$. The relations of (8-23) hold for $\psi^{(l, m)}$ too. Therefore, the right-hand side of (8-27), upon replacement of $\phi$ by $\psi$, equals

$$
\sum_{\substack{l, m \geq 0 \\ l+m \leq q-1}} a_{l, m}^{j^{\prime}}(\rho) \psi^{(l, m)}(\delta) \quad(\rho \in R, \delta \in \tilde{\Delta}),
$$

which, by induction, is in $\operatorname{Map}(\tilde{\Gamma}, \mathbb{C})^{\tilde{\Gamma}, q-1}$. Since it follows from (8-26) that the right-hand side of (8-27) with $\varphi$ replaced by $\psi$ equals $f \mid\left(\alpha_{j}-1\right)$ too, we deduce that $f \mid\left(\alpha_{j}-1\right) \in \operatorname{Map}(\tilde{\Gamma}, \mathbb{C})^{\tilde{\Gamma}, q-1}$. 
In the same way, we deduce that $f \mid\left(\alpha_{j}-1\right) \in \operatorname{Map}(\tilde{\Gamma}, \mathbb{C})^{\tilde{\Gamma}, q-1}$ when $j=t(\Gamma)$ or $j<t(\Gamma)$ and $j \neq j(1)$.

Proposition 8.12. The $\tilde{\Gamma}$-modules $\mathscr{D}_{k}(\lambda)$ and $\mathscr{D}_{k}^{\text {hol }}$ are maximally perturbable for all $k \in 2 \mathbb{Z}$ and $\lambda \in \mathbb{C}$.

Proof. It suffices to construct, for a given $f \in \mathscr{D}_{k}(\lambda)^{\tilde{\Gamma}}$, a given $q \in \mathbb{N}$, and a given $\tilde{\Gamma}$ - $q$-tuple $\boldsymbol{i}$, an element $\eta_{\boldsymbol{i}} \in \mathscr{D}_{k}(\lambda)$ such that $\eta_{\boldsymbol{i}} \mid\left(\alpha_{\boldsymbol{i}^{\prime}(1)}-1\right) \ldots\left(\alpha_{\boldsymbol{i}^{\prime}(q)}-1\right)=\delta_{\boldsymbol{i}, \boldsymbol{i}^{\prime}} f$ for all $\tilde{\Gamma}$ - $q$-tuples $\boldsymbol{j}$.

We will write $f=f_{\mathrm{cpt}}+\sum_{\kappa} f_{\kappa}$, with $\kappa$ running over a set $C$ of representatives of the $\tilde{\Gamma}$-orbits of cusps, where $f_{\mathrm{cpt}} \in\left(\mathscr{C}_{k}\right)^{\tilde{\Gamma}}$ and $f_{\kappa} \in \mathscr{D}_{k}(\lambda)^{\tilde{\Gamma}}$. We will produce perturbations for each of these components.

We choose a strict fundamental domain $\mathfrak{F}_{\tilde{\Gamma}}$ for $\tilde{\Gamma} \backslash \tilde{G}$ such that

$$
\mathfrak{F}_{\tilde{\Gamma}} \cap D_{\infty}(b)=\{(x+i y, \vartheta): 0 \leq x<1, y \geq b, 0 \leq \vartheta<\pi\}
$$

Definition 8.8 provides $b \geq A_{\Gamma}$ and $r \in \mathbb{N}$ such that $v_{\kappa}(z, \vartheta)=f\left(\tilde{g}_{\kappa}(z, \vartheta)\right)$ is in $\mathscr{E}_{k}(b, \lambda)^{\tilde{\Delta}, r}$ for each cusp $\kappa$. Furthermore, $b$ can be chosen large enough for the sets $\mathfrak{F}_{\tilde{\Gamma}} \cap D_{\kappa}(b)(\kappa \in C)$ to be pairwise disjoint. Since $f$ is $\tilde{\Gamma}$-invariant, we even have $v_{\kappa} \in \mathscr{E}_{k}(b, \lambda)^{\tilde{\Delta}}$. We choose a function $\chi \in C^{\infty}(0, \infty)$ that is equal to 0 on $\left(0, b+\frac{1}{2}\right]$ and equal to 1 on $[b+1, \infty)$, and define for $\kappa \in C$

$$
f_{\kappa}(z, \vartheta)= \begin{cases}0 & \text { if }(z, \vartheta) \in \mathfrak{F}_{\tilde{\Gamma}}-D_{\kappa}(b), \\ \chi\left(\operatorname{Im}\left(z_{1}\right)\right) v_{\kappa}\left(z_{1}, \vartheta_{1}\right) & \text { if }(z, \vartheta)=\tilde{g}_{\kappa}\left(z_{1}, \vartheta_{1}\right) \in \mathfrak{F}_{\tilde{\Gamma}} \cap D_{\kappa}(b) .\end{cases}
$$

Extend to $\tilde{G}$ by $\tilde{\Gamma}$-linearity. So $f_{\kappa}$ is equal to 0 outside $\tilde{\Gamma} D_{\kappa}(b)$, and equal to $f$ on $\tilde{\Gamma} D_{\kappa}(b+1)$. We check in Definition 8.8 that $f_{\kappa} \in \mathscr{D}_{\kappa}(\lambda)$. The function

$$
f_{\mathrm{cpt}}=f-\sum_{\kappa \in C} f_{\kappa}
$$

is $\tilde{\Gamma}$-invariant and vanishes on $D_{\kappa}(b+1)$ for all cusps $\kappa$; hence $f_{\text {cpt }} \in \mathscr{C}_{k}^{\tilde{\Gamma}}$.

Proposition 8.5 implies that there is $h_{i} \in \mathscr{C}_{k} \subset \mathscr{D}_{k}(\lambda)$ satisfying the conditions $h_{i} \mid\left(\alpha_{i(1)}-1\right) \ldots\left(\alpha_{i(q)}-1\right)=f_{\mathrm{cpt}}$ and $h_{\boldsymbol{i}^{\prime}} \mid\left(\alpha_{\boldsymbol{i}^{\prime}(1)}-1\right) \ldots\left(\alpha_{\boldsymbol{i}^{\prime}(q)}-1\right)=0$ for $\tilde{\Gamma}$ - $q$-tuples $\boldsymbol{i}^{\prime} \neq \boldsymbol{i}$. So we can restrict our attention to the $f_{\kappa}$.

Since the supports of the $f_{\kappa}$ with $\kappa \in C$ are disjoint, we can consider each of the $f_{\kappa}$ separately. Without loss of generality, we can assume that $\infty$ is a cusp of $\tilde{\Gamma}$ with $\tilde{g}_{\kappa}=1$, and take $\infty \in C$. Conjugation by the original $\tilde{g}_{\kappa}$ then gives the same result for a general $\kappa \in C$.

The function $v_{\infty}$ used in (8-28) is an element of $\mathscr{E}_{k}(b, \lambda)^{\tilde{\Delta}}$. The proof of Proposition 8.7 shows that for each $\boldsymbol{m} \in \mathbb{N}_{0}^{2}$, there is a perturbation

$$
v_{\infty}^{\boldsymbol{m}} \in \mathscr{E}_{k}(b, \lambda)^{\tilde{\Delta}, m_{1}+m_{2}+1}
$$


of $(z, \vartheta) \mapsto f_{\infty}(z, \vartheta)$ of type $\boldsymbol{m}$. We define $\eta_{i}$ by $\eta_{i}=0$ on $\tilde{G}_{b}$ and on all $\tilde{\Gamma} D_{\kappa}(b)$ for all $\kappa \in C \backslash\{\infty\}$, and

$$
\eta_{i}(\rho(x+i y, \vartheta))=\sum_{\substack{l, m \geq 0 \\ l+m \leq q}} \chi(y) a_{l, m}^{i}(\rho) v_{\infty}^{(l, m)}(x+i y, \vartheta)
$$

for $y \geq b$ and $\rho$ in a system of representatives $R$ of $\tilde{\Gamma} / \tilde{\Delta}$. The functions $a_{l, m}^{i}$ are as in (8-24). Since the sets $\rho D_{\infty}(b)$ are disjoint, this defines a smooth function, which can be checked to be an element of $\mathscr{D}_{k}(\lambda)$.

For each fixed $g=(x+i y, \vartheta)$ with $y \geq b$, the function $\delta \mapsto v_{\infty}^{(l, m)}(\delta g)$ on $\tilde{\Delta}$ satisfies the same relations as $\delta \mapsto \varphi^{(l, m)}(\delta) v_{\infty}(g)$ in (8-23). So their difference, as a function of $\delta$, satisfies (8-25).

Ignoring smoothness for a moment, we have $f_{\infty} \in \operatorname{Map}(\tilde{G}, \mathbb{C})^{\tilde{\Gamma}}$. Equation (8-9) gives a function $h_{i}$ on $\tilde{G}$ such that $h_{i} \mid\left(\alpha_{i^{\prime}(1)}-1\right) \ldots\left(\alpha_{i^{\prime}(q)}-1\right)=\delta_{i, i^{\prime}} f_{\infty}$ for all $\tilde{\Gamma}$ - $q$-tuples $\boldsymbol{i}^{\prime}$. With our choice of fundamental domain, and using (8-24), we find for $\rho \in R, \delta \in \tilde{\Delta}$, and $g=(x+i y, \vartheta)$ with $y \geq b$ :

$$
h_{i}(\rho \delta g)=\sum_{\substack{l, m \geq 0 \\ l+m \leq q}} a_{l, m}^{i}(\rho) \varphi^{(l, m)}(\delta) \chi(y) v_{\infty}(g) .
$$

Outside $\tilde{\Gamma} D_{\infty}(b)$, the functions $\stackrel{l+m \leq q}{f_{\infty}}, h_{i}$ are zero. With Lemma 8.11, we conclude that the function induced by

$$
\left(\eta_{i}-h_{i}\right)(\rho \delta g)=\sum_{\substack{l, m \geq 0 \\ l+m \leq q}} a_{l, m}^{i}(\rho) \chi(y)\left(v_{\infty}^{(l, m)}(\delta g)-\varphi^{(l, m)}(\delta) v_{\infty}(g)\right)
$$

is in $\operatorname{Map}(\tilde{G}, \mathbb{C})^{\tilde{\Gamma}, q}$. This implies that

$$
\eta_{\boldsymbol{i}} \in\left(h_{\boldsymbol{i}}+\operatorname{Map}(\tilde{G}, \mathbb{C})^{\tilde{\Gamma}, q}\right) \cap \mathscr{D}_{k}(\lambda)=\mathscr{D}_{k}(\lambda)^{\tilde{\Gamma}, q+1},
$$

and behaves in the desired way under $\left(\alpha_{i^{\prime}(1)}-1\right) \ldots\left(\alpha_{i^{\prime}(q)}-1\right)$ for all $\tilde{\Gamma}$ - $q$-tuples $\boldsymbol{i}^{\prime}$. Thus, we have proved that $\mathscr{D}_{k}(\lambda)$ is maximally perturbable.

Everywhere in this proof, we can replace $\mathscr{E}_{k}(b, \lambda)$ by $\mathscr{E}_{k}^{\text {hol }}(b)$, and $\mathscr{D}_{k}(\lambda)$ by $\mathscr{D}_{k}^{\text {hol }}$. In that way, we also obtain the maximal perturbability of $\mathscr{D}_{k}^{\text {hol }}$, thus completing the proof of Proposition 8.12.

8D3. Relations between the spaces $\mathscr{C}_{k}$ and $\mathscr{D}_{k}(\lambda)$. By Remark 8.10 , for each $f \in \mathscr{D}_{k}(\lambda)$, the support of $(\omega-\lambda) f$ is contained in some set $\tilde{G}_{b}$, and hence $(\omega-\lambda) f \in \mathscr{C}_{k}$. So the differential operator $\omega-\lambda$ maps $\mathscr{D}_{k}(\lambda)$ to $\mathscr{C}_{k}$. Since the operator $\omega$ commutes with the action of $\tilde{\Gamma}$, we have $(\omega-\lambda) \mathscr{D}_{k}(\lambda)^{\tilde{\Gamma}, q} \subset \mathscr{C}_{k}^{\tilde{\Gamma}, q}$ for all $q \geq 1$. Similarly, $\boldsymbol{E}^{-}\left(\mathscr{D}_{k}^{\text {hol }}\right)^{\tilde{\Gamma}, q} \subset \mathscr{C}_{k-2}^{\tilde{\Gamma}, q}$ for all $q \geq 1$.

Proposition 8.13. Let $\lambda \in \mathbb{C}$ and $k \in 2 \mathbb{Z}$. The following maps are surjective:

i) $\omega-\lambda: \mathscr{D}_{k}(\lambda)^{\tilde{\Gamma}} \rightarrow \mathscr{C}_{k}^{\tilde{\Gamma}}$, 

ii) $\boldsymbol{E}^{-}:\left(\mathscr{D}_{k}^{\mathrm{hol}}\right)^{\tilde{\Gamma}} \rightarrow \mathscr{C}_{k-2}^{\tilde{\Gamma}}$.

Before presenting the proof we give a corollary:

Corollary 8.14. For each $q \geq 1$, the maps $\omega-\lambda: \mathscr{D}_{k}\left(\lambda_{s}\right)^{\tilde{\Gamma}, q} \rightarrow \mathscr{C}_{k}^{\tilde{\Gamma}, q}$ and $\boldsymbol{E}^{-}$: $\left(\mathscr{D}_{k}^{\text {hol }}\right)^{\tilde{\Gamma}, q} \rightarrow \mathscr{C}_{k}^{\tilde{\Gamma}, q}$ are surjective.

Proof. Proposition 8.13 gives the case $q=1$. The rows in the following commutative diagram are exact by Propositions 8.5 and 8.12. See (5-11) for $\mathrm{m}_{q}$.

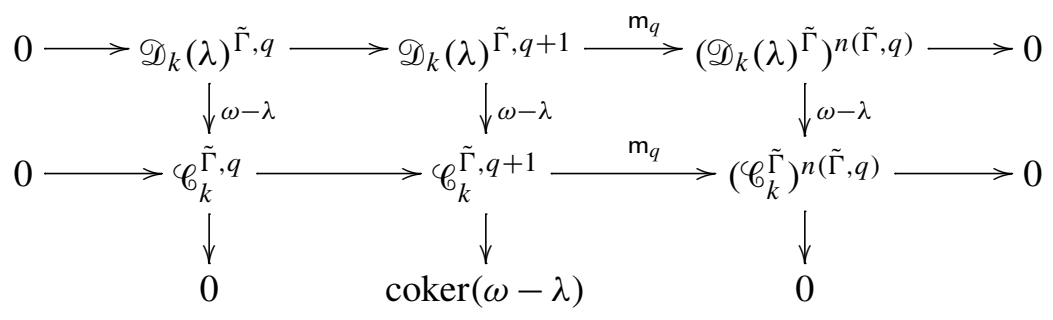

The third column is exact by Proposition 8.13. With the exactness of the first column as induction hypothesis, we obtain the vanishing of $\operatorname{coker}(\omega-\lambda)$, and thus the surjectivity of $\omega-\lambda: \mathscr{D}_{k}(\lambda)^{\tilde{\Gamma}, q+1} \rightarrow \mathscr{C}_{k}^{\tilde{\Gamma}, q+1}$, by the snake lemma.

The case of $\boldsymbol{E}^{-}:\left(\mathscr{D}_{k}^{\text {hol }}\right)^{\tilde{\Gamma}, q} \rightarrow \mathscr{C}_{k}^{\tilde{\Gamma}, q}$ is similar.

8D4. Proof of Proposition 8.13. We first note that the spaces $\mathscr{D}_{k}(\lambda)^{\tilde{\Gamma}}$ and $\mathscr{C}_{k}^{\tilde{\Gamma}}$ are invariant under $\tilde{Z}$. Hence, the weight $k$ is strict and we are dealing with functions on $G=\mathrm{PSL}_{2}(\mathbb{R})$. (See the first statement in Theorem 6.4.) We use the spectral theory of automorphic forms to prove Proposition 8.13.

We work with the space of square integrable functions on $\tilde{\Gamma} \backslash \tilde{G}=\Gamma \backslash G$ of strict weight $k \in 2 \mathbb{Z}$, where $G=\mathrm{PSL}_{2}(\mathbb{R})$. We can view the elements of the Hilbert space $H_{k}=L^{2}(\tilde{\Gamma} \backslash \tilde{G})_{k}=L^{2}(\Gamma \backslash G)_{k}$ as functions $z \mapsto f(z, 0)$ on $\mathfrak{H}$, transforming according to weight $k$ as indicated in (4-7). The inner product in $H_{k}$ is given by

$$
\left(f, f_{1}\right)=\int_{\mathfrak{F}} f(z, 0) \overline{f_{1}(z, 0)} \frac{d x d y}{y^{2}} .
$$

Here $\mathfrak{F}$ can be any fundamental domain for $\Gamma \backslash \mathfrak{H}$. We take it so that for each $b>A_{\Gamma}$, it has a decomposition

$$
\mathfrak{F}=\mathfrak{F}_{b} \sqcup \bigsqcup_{\kappa \in C} V_{\kappa}, \quad V_{\kappa}=\left\{g_{\kappa}(x+i y): x_{\kappa} \leq x \leq x_{\kappa}+1, y \geq b\right\},
$$

with $C$ a system of representatives of the $\Gamma$-orbits of cusps, and $x_{\kappa} \in \mathbb{R}$ depending on $\mathfrak{F}$ and on the earlier choice of the $g_{\kappa}$. The set $\mathfrak{F}_{b}$ has compact closure in $\mathfrak{H}$.

The differential operator $\omega_{k}=-y^{2} \partial_{y}^{2}-y^{2} \partial_{x}^{2}+i k y \partial_{x}$ in (4-8) determines a densely defined self-adjoint operator $A_{k}$ in $H_{k}$. The spectral theory of automorphic forms gives the decomposition of this operator $A_{k}$ in terms of Maass forms. One 
may consult Chapters 4 and 7 in [Iwaniec 1995] for weight 0. For other weights, the proofs are almost completely similar. (See [Roelcke 1966; 1967].)

The spectral decomposition gives the Parseval formula

$$
\left(f, f_{1}\right)=\sum_{\ell} a_{k}^{\ell}(f) \overline{a_{k}^{\ell}\left(f_{1}\right)}+\sum_{\kappa} \frac{1}{2 \pi} \int_{0}^{\infty} e_{k}^{\kappa}(f ; i t) \overline{e_{k}^{\kappa}\left(f_{1} ; i t\right)} d t,
$$

with $\kappa$ running through a set of representatives of the cuspidal orbit and $\ell$ indexing a maximal orthonormal system of eigenfunctions $\psi_{k}^{\ell}$ of $A_{k}$, with eigenvalue $\lambda_{\ell} \in \mathbb{R}$. (These eigenvalues are discrete in $\mathbb{R}$, with finite multiplicities.) For each $f \in H_{k}$, we have $a_{k}^{\ell}(f)=\left(f, \psi_{k}^{\ell}\right)$. If $f$ is sufficiently regular, then the functions $e_{k}^{\kappa}(f ; \cdot)$ are obtained by integration against the Eisenstein series $E_{k}^{\kappa}(i t)$ at the cusp $\kappa$. The Parseval formula (8-34) shows that the Hilbert space $H_{k}$ is isomorphic to the direct sum of the subspace spanned by the $\psi_{k}$ and a number of copies of $L^{2}((0, \infty), d t / 2 \pi)$. The operator $A_{k}$ corresponds to a multiplication operator. For $f$ in its domain, we have $a_{k}^{\ell}\left(A_{k} f\right)=\lambda_{k} a_{k}(f)$ and

$$
e_{k}^{\kappa}\left(A_{k} f ; i t\right)=\left(\frac{1}{4}+t^{2}\right) e_{k}^{\kappa}(t ; i t)
$$

For the smooth, modulo $\Gamma$ compactly supported elements $f \in \mathscr{C}_{k}^{\tilde{\Gamma}}$, we have $a_{k}^{\ell}(f)=\mathrm{O}\left(\left(\left|\lambda_{k}\right|+1\right)^{-a}\right)$ and $e_{k}^{\kappa} \kappa(f ; i t)=\mathrm{O}\left((1+t)^{-1}\right)$ for each $a \in \mathbb{R}$. Moreover, $e_{k}(f ; \cdot)$ extends as a holomorphic function on some neighborhood of $i \mathbb{R}$ in $\mathbb{C}$.

Solving $\left(A_{k}-\lambda\right) f_{1}=f$ with unknown $f_{1} \in H_{k}$ for a given $f \in \mathscr{C}_{k}^{\Gamma}$ can be done by dividing by the factor $\lambda_{k}-\lambda$, respectively $\frac{1}{2}+i t^{2}-\lambda_{k}$ in the spectral decomposition, if these divisions are possible. The requirements are:

i) $a_{k}^{\ell}(f)=0$ if $\lambda_{\ell}=\lambda$. This condition occurs for at most finitely many indices $\ell$.

ii) $e_{k}^{\kappa}\left(t ; i t_{\lambda}\right)=0$ for all $\kappa$ if $\lambda=\frac{1}{4}+t_{\lambda}^{2}$ with $t_{\lambda} \in(0, \infty)$.

iii) $s \mapsto e_{k}^{\kappa}(f ; s)$ has a double zero at $s=0$ for all $\kappa$ if $\lambda=\frac{1}{4}$.

These requirements impose finitely many linear conditions on $f$. So there is a subspace $\mathscr{C}_{k}(\Gamma, \lambda)$ of $\mathscr{C}_{k} \Gamma$ such that for $f \in \mathscr{C}_{k}(\Gamma, \lambda)$, the equation $\left(A_{k}-\lambda\right) f_{1}=f$ can be solved with $f_{1} \in H_{k}$. This means that $(\omega-\lambda) f_{1}=f$ holds in the sense of distributions, and hence $f_{1}$ is in $C^{\infty}(\mathfrak{H})$ with the transformation behavior (4-7). It need not be in $\mathscr{C}_{k} \Gamma$. However, we have $\omega_{k} f_{1}=\lambda k_{1}$ on the sets $\left\{g_{\kappa} z \in \mathfrak{H}: \operatorname{Im} z>b\right\}$ for some $b$ depending on the support of $f$. The square integrability of $f_{1}$ ensures that it has less than exponential growth at the cusps, and hence $f_{1} \in \mathscr{D}_{k}(\lambda)^{\Gamma}$.

So we are done with the proof of part i) for the subspace $\mathscr{C}_{k}(\Gamma, \lambda)$ of $\mathscr{C}_{k}^{\tilde{\Gamma}}$ of finite codimension.

Lemma 8.15. Let $\kappa$ be the cusp that we keep fixed. Suppose that $\lambda$ is in the spectrum of $A_{k}$. Then there is a finite set $X \subset \mathbb{Z}$ such that, for each $n \in X$, there exist $h_{n} \in \mathscr{C}_{k}^{\tilde{\Gamma}}$ 
of the form

$$
h_{n}\left(\gamma \tilde{g}_{\kappa}(z, \vartheta)\right)= \begin{cases}e^{2 \pi i n x} \chi_{n}(y) e^{i k \vartheta} & \text { on } \tilde{\Gamma} D_{\kappa}\left(A_{\Gamma}\right) \\ 0 & \text { elsewhere }\end{cases}
$$

for some $\chi_{n} \in C_{c}^{\infty}\left(A_{\Gamma}, \infty\right)$, such that $\left\{h_{n}+C_{k}(\Gamma, \lambda)\right\}_{n} \operatorname{spans} \mathscr{C}_{k}^{\tilde{\Gamma}} / \mathscr{C}_{k}(\Gamma, \lambda)$.

Proof. We shall examine each of the three cases for the eigenvalues of $A_{k}$ on $H_{k}$ separately:

- $\lambda=\frac{1}{4}-s^{2} \notin\left[\frac{1}{4}, \infty\right)$. Assume $\operatorname{Re} s>0$. There are finitely many indices $\ell_{1}, \ldots, \ell_{m}$ such that $\lambda_{\ell_{j}}=\lambda$. The $\psi_{k}^{\ell_{j}}$ form a basis of $\operatorname{ker}\left(A_{k}-\lambda\right)$. Each of these $m$ linearly independent square integrable automorphic forms is given by its Fourier expansion at the fixed cusp $\kappa$. By Proposition 7.1, the Fourier terms of nonzero order are multiples of $\omega_{k}(n, s)$. The Fourier term of order zero is a multiple of $y^{1 / 2-s} e^{i k \vartheta}$. We choose a set $X$ of $m$ elements in $\mathbb{Z}$ such that the $m \times m$-matrix whose columns are the $n$-th Fourier coefficients of $\psi_{k}^{\ell_{j}}(1 \leq j \leq m)$, with $n \in X$, is invertible. We choose the $\chi_{n} \in C_{c}^{\infty}, n \in X$, in the statement of the lemma, in such a way that $\int_{A_{\Gamma}}^{\infty} \chi_{n}(y) \overline{\omega_{k}(n, s)(i y, 0)} d y / y^{2} \neq 0$ or $\int_{A_{\Gamma}}^{\infty} \chi_{n}(y) \overline{y^{1 / 2-s}} d y / y^{2} \neq 0$, as the case may be. Consider the linear form on the space $A_{k}^{2}(\lambda)$ of square integrable automorphic forms with eigenvalue $\lambda$ given by

$$
\begin{aligned}
\psi \mapsto\left(h_{n}, \psi\right)= & \int_{\mathfrak{F}} h_{n}(z, 0) \overline{\psi(z, 0)} \frac{d x d y}{y^{2}} \\
= & \int_{A_{\Gamma}}^{\infty} \int_{-1 / 2}^{1 / 2} \chi_{n}(y) e^{2 \pi i n x} \bar{a}_{0} y^{1 / 2-\bar{s}} \frac{d x d y}{y^{2}} \\
& +\sum_{m \neq 0} \bar{a}_{m} \int_{A_{\Gamma}}^{\infty} \int_{-1 / 2}^{1 / 2} \chi_{n}(y) e^{2 \pi i n x} \overline{\omega_{k}(m, s)(i y, 0)} \frac{d x d y}{y^{2}} .
\end{aligned}
$$

This depends only on the Fourier coefficient of $\psi$ of order $n$ in the expansion at $\kappa$. Therefore, the $m \times m$-matrix with the scalar product $\left(h_{n}, \psi_{k}^{\ell_{j}}\right)$ at position $(j, n)$ is invertible. (Here $j$ runs from 1 to $m$, and $n$ runs through $X$.) Hence, there are complex numbers $b_{j, p}($ with $1 \leq j \leq m, p \in X)$ such that $\sum_{n \in X} b_{j, n}\left(h_{n}, \psi_{k}^{\ell_{j^{\prime}}}\right)=\delta_{j, j^{\prime}}$. Setting

$$
c_{n}(f)=\sum_{j^{\prime}=1}^{m}\left(f, \psi_{k}^{\ell_{j^{\prime}}}\right) b_{j^{\prime}, n}
$$

for $f \in \mathscr{C}_{k}^{\tilde{\Gamma}}$, we obtain for $1 \leq j \leq m$ :

$$
\sum_{n \in X} c_{n}(f)\left(h_{n}, \psi^{\ell_{j}}\right)=\left(f, \psi_{k}^{\ell_{j}}\right) .
$$

So $f-\sum_{n} c_{n}(f) h_{n}$ is indeed in $\mathscr{C}_{k}(\Gamma, \lambda)$. 
- $\lambda=\frac{1}{4}+t^{2}, t \in \mathbb{R} \backslash\{0\}$. A basis of $\operatorname{ker}\left(A_{k}-\lambda\right)$ in this case consists of Eisenstein series $E_{k}^{v}(i t, \cdot)(v \in C)$ and possibly cusp forms $\psi_{k}^{\ell_{j}}$ with $\lambda_{\ell_{j}}=\lambda$. The proof of the previous case can be applied with the obvious adjustments (for example, replacing scalar products by integrals for the terms corresponding to $E_{k}^{v}$ ) to give the result. The only essential modification is that we have to use the space $A_{k}^{*}(\lambda)$ of automorphic forms with polynomial growth and eigenvalue $\lambda$ in place of $A_{k}^{2}(\lambda)$, because the Eisenstein series are not square integrable. This can be done because (conjugates of) elements of $A_{k}^{*}(\lambda)$ appear only integrated against elements of $\mathscr{C}_{k}^{\tilde{\Gamma}}$, which have compact support modulo $\tilde{\Gamma}$.

- $\lambda=\frac{1}{4}$. Now we have the condition that $e_{k}^{\kappa}\left(f-\sum_{n} h_{n} ; i t\right)$ should have a double zero at $t=0$ or, equivalently, that the first two terms of the Taylor expansion at $s=0$ should vanish. Since the first two Taylor terms of $E_{k}^{\kappa}(-; z)$ are linearly independent from the other functions in $A_{k}^{*}\left(\frac{1}{4}\right)$, a choice of $\chi_{n}$ with the desired properties is again possible.

Now we turn to the task of solving $\left(\omega-\lambda_{s}\right) f_{1}=h_{n}$ with $f_{1} \in \mathscr{D}_{k}(\lambda)^{\tilde{\Gamma}}$ for $h_{n}$ as in Lemma 8.15. We aim at $f_{1}$ with support in $\tilde{\Gamma} D_{\kappa}\left(A_{\Gamma}\right)$. Writing

$$
f_{1}\left(\tilde{g}_{\kappa}(z, \vartheta)\right)=e^{2 \pi i n x} h(y) e^{i k \vartheta},
$$

the differential equation $(\omega-\lambda) f_{1}=h_{n}$ becomes

$$
-y^{2} h^{\prime \prime}(y)+\left(4 \pi^{2} n^{2} y^{2}-2 \pi n k y-\frac{1}{4}+s^{2}\right) h(y)=\chi_{n}(y) .
$$

(Compare (7-3).) This ordinary differential equation is regular on $y \geq A_{\Gamma}$. It has a unique solution for the initial conditions $h\left(A_{\Gamma}\right)=h^{\prime}\left(A_{\Gamma}\right)=0$. It is zero below the support of $\chi_{n}$. Since $\chi_{n}$ has compact support, the function $h$ thus obtained is a solution of the homogeneous Equation (7-3) on $(b, \infty)$ for some $b>A_{\Gamma}$ depending on $\operatorname{Supp}\left(\chi_{n}\right)$. Thus, we see that $(z, \vartheta) \mapsto f_{1}\left(\tilde{g}_{\kappa}(z, \vartheta)\right)$ is an element of $\mathscr{W}_{k}(n, s)$. Hence, it may have exponential growth of order $e^{(2 \pi|n|+\delta) y}$. This is the point where the need to work with exponentially growing functions arises.

We extend $f_{1}$ by $\tilde{\Gamma}$-invariance, and check that it is an element of $\mathscr{D}_{k}\left(\lambda_{s}\right)$. This completes the proof of the first statement in Proposition 8.13.

Let us denote by $\boldsymbol{E}_{k}^{-}$the unbounded operator $H_{k} \rightarrow H_{k-2}$ given by the differential operator $\boldsymbol{E}^{-}$, and similarly $\boldsymbol{E}_{k-2}^{+}: H_{k-2} \rightarrow H_{k}$. For the surjectivity of $\boldsymbol{E}^{-}:\left(\mathscr{D}_{k}^{\mathrm{hol}}\right)^{\tilde{\Gamma}} \rightarrow \mathscr{C}_{k-2}^{\tilde{\Gamma}}$, we first note that, on an eigenfunction of $\omega$ in weight $k-2$ with eigenvalue $\lambda$, the operator $\boldsymbol{E}_{k}^{-} \boldsymbol{E}_{k-2}^{+}$acts as multiplication by $-4\left(\lambda-\frac{k}{2}+\frac{k^{2}}{4}\right)$. See (5-5). We will use $\boldsymbol{E}_{k-2}^{+}$to "invert" $\boldsymbol{E}_{k}^{-}$.

We can arrange the choice of the orthonormal systems of square integrable eigenfunctions in $H_{k-1}$ and $H_{k}$ in such a way that $\boldsymbol{E}^{-} \psi_{k}^{\ell}=-\sqrt{k^{2}-2 k+4 \lambda_{k}} \psi_{k-2}^{\ell}$. We have $k^{2}-2 k+4 \lambda_{k} \geq 0$. This factor can be zero for finitely many $\ell$, corresponding to a system of holomorphic automorphic forms of weight $k$. For these $\ell$, there is no 
corresponding eigenfunction $\psi_{k-2}^{\ell}$. Also there can be finitely many indices $\ell$ such that $\psi_{k-2}^{\ell}$ does not occur as image of some $\psi_{k-2}^{\ell}$, corresponding to antiholomorphic automorphic forms. Anyhow, this leads to $a_{k}^{\ell}\left(\boldsymbol{E}^{+} f\right)=\sqrt{k^{2}-2 k+4 \lambda_{\ell}} a_{k-2}^{\ell}(f)$, with $k^{2}-2 k+4 \lambda_{\ell} \neq 0$ except for finitely many $\ell$. For the Eisenstein series, we have $e_{k}^{\kappa}\left(\boldsymbol{E}^{+} f ; s\right)=(2 s+k-1) e_{k-2}^{\kappa}(f, s)$. Here the factor is nonzero for all $s \in i[0, \infty)$. So for $f$ in a subspace of finite codimension in $\mathscr{C}_{k-2}^{\tilde{\Gamma}}$, we can find by the method used for the first part a smooth element $f_{1} \in H_{k}$ with $\boldsymbol{E}^{-} f_{1}=f$. It is smooth, and near all cusps it is annihilated by $\boldsymbol{E}^{-}$, and hence it is holomorphic near the cusps. So it is in $\left(\mathscr{D}_{k}^{\text {hol }}\right)^{\tilde{\Gamma}}$.

We are left with finitely many $\ell$ for which $\boldsymbol{E}_{k-2}^{+} \psi_{k-2}^{\ell}=0$. We form functions $h_{n}$ as in Lemma 8.15, corresponding to a set $X$ of Fourier term orders such that elements of $H_{k-2}^{a}$ are determined by the Fourier coefficients in $X$. Solving $\boldsymbol{E}_{k}^{-} f_{1}=h_{n}$ leads to the differential equation

$$
\begin{gathered}
\left(-2 i y \partial_{x}+2 y \partial_{y}-k\right) e^{2 \pi i n x} \varphi(y)=\chi(y), \\
\varphi\left(y_{0}\right)=\varphi^{\prime}\left(y_{0}\right)=0,
\end{gathered}
$$

with which we proceed as in the previous case.

This establishes the surjectivity of $\boldsymbol{E}^{-}:\left(\mathscr{D}_{k}^{\text {hol }}\right)^{\tilde{\Gamma}} \rightarrow \mathscr{C}_{k-2}^{\tilde{\Gamma}}$ in Proposition 8.13.

8E. Higher-order invariants and Maass forms. We now will derive the main results of this paper, Theorems 6.5 and 6.8 , from the following result:

Proposition 8.16. The $\tilde{\Gamma}$-modules

$$
\tilde{\mathscr{E}}_{k}^{\prime}(\lambda):=\operatorname{ker}\left(\omega-\lambda: \mathscr{D}_{k}(\lambda) \rightarrow \mathscr{C}_{k}\right)
$$

and

$$
\mathscr{H}_{k}^{\prime}:=\operatorname{ker}\left(\boldsymbol{E}^{-}: \mathscr{D}_{k}^{\text {hol }} \rightarrow \mathscr{C}_{k-2}\right)
$$

are maximally perturbable.

Proof. We have the following extension of the commutative diagram (8-32):

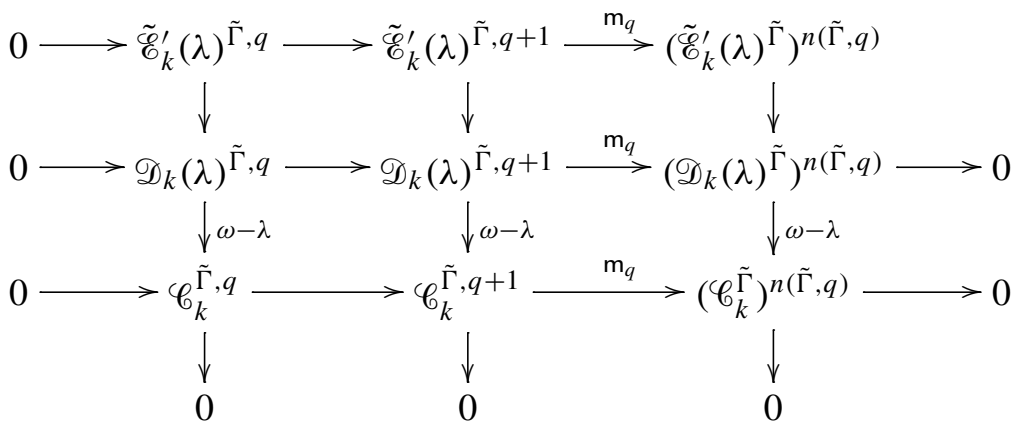


The exactness of the columns follows from the definition of $\tilde{\mathscr{E}}_{k}^{\prime}(\lambda),(3-2)$, the leftexactness of the functor hom $\mathbb{C}[\Gamma]\left(I^{q} \backslash \mathbb{C}[\Gamma],-\right)$, and Corollary 8.14. Propositions 8.3 and 8.12 imply that the second and third row are exact. The snake lemma then implies that the first row is exact and that

$$
\mathrm{m}_{q}: \tilde{\mathscr{E}}_{k}^{\prime}(\lambda)^{\tilde{\Gamma}, q+1} \rightarrow\left(\tilde{\mathscr{E}}_{k}^{\prime}(\lambda)^{\tilde{\Gamma}}\right)^{n(\tilde{\Gamma}, q)}
$$

is surjective.

Replacing in this diagram the space $\tilde{\mathscr{E}}_{l}^{\prime}(\lambda)$ by $\mathcal{H}_{k}^{\prime}$ and the map $\omega-\lambda$ by $\boldsymbol{E}^{-}$, we obtain the maximal perturbability of $\mathscr{H}_{k}^{\prime}$.

Proof of Theorems 6.5 and 6.8. The $\tilde{\Gamma}$-module $\tilde{\mathscr{E}}_{k}^{\prime}(\lambda)$ is contained in $\tilde{\mathscr{E}}_{k}(\tilde{\Gamma}, \lambda)$. See Definition 6.3. It is a smaller space than $\tilde{\mathscr{E}}_{k}(\tilde{\Gamma}, \lambda)$, since elements of $\mathscr{D}_{k}(\lambda)$ have a special structure near the cusps. With $(8-22), \tilde{\mathscr{E}}_{k}(\tilde{\Gamma}, \lambda)^{\tilde{\Gamma}}$ is a subspace of $\tilde{\mathscr{E}}_{k}^{\prime}(\lambda)^{\tilde{\Gamma}}$. Therefore $\tilde{\mathscr{C}}_{k}(\tilde{\Gamma}, \lambda)^{\tilde{\Gamma}}=\tilde{\mathscr{E}}_{k}^{\prime}(\lambda)^{\tilde{\Gamma}}$, and thus

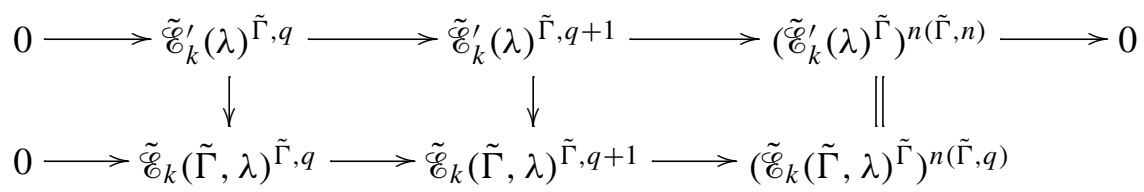

with exact rows. Induction with respect to $q$ and the snake lemma show that $\tilde{\mathscr{E}}_{k}(\tilde{\Gamma}, \lambda)^{\tilde{\Gamma}, q}$ is equal to $\tilde{\mathscr{E}}_{k}^{\prime}(\lambda)^{\tilde{\Gamma}, q}$ for all $q$. Hence, the space $\tilde{\mathscr{E}}_{k}(\tilde{\Gamma}, \lambda)$ is maximally perturbable.

The proof of Theorem 6.8 is completely similar.

\section{Index of commonly used notation}

\begin{tabular}{|c|c|c|c|c|c|}
\hline$(y)$ & Section $5 \mathrm{~A}$ & $E_{k}(\Gamma, \lambda)$ & Definition 4.1 & $\tilde{G}_{a}$ & 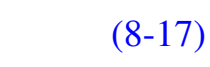 \\
\hline$a_{k}^{\ell}(f)$ & $(8-34)$ & $E_{k}^{\mathrm{hol}}\left(\Gamma, \lambda_{k}\right)$ & Section 4B & $h_{i}$ & $8-9),(8-10)$ \\
\hline & Section 5B & $\boldsymbol{E}^{ \pm}$ & $(5-4)$ & $H_{i}$ & Section 3B \\
\hline${ }_{i}{ }_{i}$ & Section $5 C$ & $e_{k}^{\kappa}(f ; i t)$ & $(8-34)$ & $h_{k}^{\boldsymbol{m}}(n, s)$ & $(7-11)$ \\
\hline$b(\boldsymbol{i})$ & $(3-8),(5-7)$ & \multicolumn{2}{|c|}{$\begin{array}{r}\mathscr{E}_{k}\left(y_{0}, \lambda\right), \mathscr{\mathscr { E }}_{k}^{\mathrm{hol}}\left(y_{0}\right) \\
\text { Definition } 8.6\end{array}$} & \multicolumn{2}{|c|}{$\mathscr{H}_{k}(\tilde{\Gamma}), \mathscr{H}_{k}^{p}(\tilde{\Gamma}), \mathscr{H}_{k}^{c}(\tilde{\Gamma})$} \\
\hline$C_{k}^{\infty}(\tilde{G})$ & $(8-11)$ & $\tilde{\mathscr{E}}_{k}(\tilde{\Gamma}, \lambda) \quad \mathrm{I}$ & Definition 6.3 & $\eta_{i}$ & Section $5 C$ \\
\hline $\mathscr{C}_{k}$ & Definition 8.4 & $\tilde{E}_{r}(\tilde{\Gamma}, \chi, \lambda)$ & Definition 6.3 & $\eta_{r}(n ; z, \vartheta)$ & $(7-16$ \\
\hline$D_{\kappa}(a)$ & $(8-16)$ & $E_{k}(\Gamma, \lambda)$ & Definition 4.1 & $\eta_{k}(n)$ & $(7-17)$ \\
\hline $\mathscr{D}_{k}(\lambda), \mathscr{D}_{k}^{\text {hol }}$ & ${ }^{1}$ Definition 8.8 & $f_{i}$ & $(8-2)$ & $\eta_{k}^{\boldsymbol{m}}(n ; z, \vartheta)$ & $(7-18)$ \\
\hline 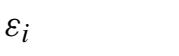 & Section $5 C$ & $g_{\kappa}$ & $(4-1)$ & $k(\vartheta)$ & Section $5 A$ \\
\hline$t_{i}$ & ection 3B & $\tilde{G}$ & Section $5 \mathrm{~A}$ & $\kappa_{i}$ & Section $5 C$ \\
\hline
\end{tabular}




$\begin{array}{lrlrlr}L_{k} & (4-3) & n(x) & \text { Section 5A } & t & \text { Section 5B } \\ L(z, \vartheta) & (6-3) & n(\Gamma, q) & (3-4) & t(\Gamma) & \text { Section 3B } \\ \mathrm{m}_{q} & (3-3) & P_{i} & \text { Section 3B } & \mathscr{V}_{k}(n, s), \mathscr{V}_{k}^{0}(n, s) \\ M_{k}(\Gamma, \lambda) & \text { Definition 4.1 } & \mathrm{pr}, \mathrm{pr}_{2} & \text { Section 5A } & \mathscr{W}_{r}(v, s) & \text { Definition 7.2 } \\ M_{k}(\Gamma, \lambda) & \text { Definition 4.1 } & \pi_{i} & \text { Section 5C } & W & (7-2) \\ M_{k}^{\text {hol }}\left(\Gamma, \lambda_{k}\right) & \text { Section 4B } & Q_{n} & (7-10) & \zeta & \text { Section 5B } \\ \mu_{f} & (3-5) & s & \text { Section 5B } & \omega & (5-5) \\ n_{\mathrm{ell}}, n_{\mathrm{par}} & \text { Section 3B } & S\left(y_{0}\right) & (8-19) & \omega_{r}, \hat{\omega}_{r} & (7-4)\end{array}$

\section{References}

[Bruggeman 1986] R. W. Bruggeman, "Modular forms of varying weight, III", J. Reine Angew. Math. 371 (1986), 144-190. MR 88e:11040 Zbl 0588.10021

[Bruggeman 1994] R. W. Bruggeman, Families of automorphic forms, Monographs in Mathematics 88, Birkhäuser, Boston, MA, 1994. MR 95k:11060 Zbl 0821.11029

[Bruinier et al. 2008] J. H. Bruinier, K. Ono, and R. C. Rhoades, "Differential operators for harmonic weak Maass forms and the vanishing of Hecke eigenvalues", Math. Ann. 342:3 (2008), 673-693. MR 2009f:11046 Zbl 05375997

[Chen 1971] K.-t. Chen, "Algebras of iterated path integrals and fundamental groups", Trans. Amer. Math. Soc. 156 (1971), 359-379. MR 43 \#1069 Zbl 0217.47705

[Chinta et al. 2002] G. Chinta, N. Diamantis, and C. O'Sullivan, "Second order modular forms", Acta Arith. 103:3 (2002), 209-223. MR 2003b:11037 Zbl 1020.11025

[Deitmar 2008] A. Deitmar, "Higher order invariants, cohomology, and automorphic forms", preprint, 2008. arXiv 0811.1088v1

[Deitmar 2009] A. Deitmar, "Higher order group cohomology and the Eichler-Shimura map", $J$. Reine Angew. Math. 629 (2009), 221-235. MR 2011a:11107 Zbl 05551467

[Diamantis 1999] N. Diamantis, "Special values of higher derivatives of $L$-functions", Forum Math. 11:2 (1999), 229-252. MR 2000a:11075 Zbl 0932.11032

[Diamantis and O'Sullivan 2008] N. Diamantis and C. O'Sullivan, "The dimensions of spaces of holomorphic second-order automorphic forms and their cohomology", Trans. Amer. Math. Soc. 360:11 (2008), 5629-5666. MR 2009h:11067 Zbl 1158.11021

[Diamantis and Sim 2008] N. Diamantis and D. Sim, "The classification of higher-order cusp forms", J. Reine Angew. Math. 622 (2008), 121-153. MR 2010a:11067 Zbl 1230.11049

[Diamantis and Sreekantan 2006] N. Diamantis and R. Sreekantan, "Iterated integrals and higher order automorphic forms", Comment. Math. Helv. 81:2 (2006), 481-494. MR 2007b:11050 Zbl 1156.11019

[Goldfeld 1995] D. Goldfeld, "Special values of derivatives of $L$-functions", pp. 159-173 in Number theory (Halifax, 1994), edited by K. Dilcher, CMS Conf. Proc. 15, American Mathematical Society, Providence, RI, 1995. MR 96h:11039 Zbl 0845.11021

[Iwaniec 1995] H. Iwaniec, Introduction to the spectral theory of automorphic forms, Revista Matemática Iberoamericana, Madrid, 1995. MR 96f:11078 Zbl 0847.11028 
[Koecher and Krieg 1998] M. Koecher and A. Krieg, Elliptische Funktionen und Modulformen, Springer, Berlin, 1998. MR 2000e:11047 Zbl 0895.11001

[Kra 1969] I. Kra, “On cohomology of kleinian groups", Ann. of Math. (2) 89 (1969), 533-556. MR 41 \#8656a Zbl 0193.04003

[Lang 1975] S. Lang, $\mathrm{SL}_{2}$ (R), Addison-Wesley, Reading, MA, 1975. MR 55 \#3170 Zbl 0311.22001

[Lehner 1964] J. Lehner, Discontinuous groups and automorphic functions, Mathematical Surveys 8 , American Mathematical Society, Providence, R.I., 1964. MR 29 \#1332 Zbl 0178.42902

[Maass 1983] H. Maass, Lectures on modular functions of one complex variable, 2nd ed., Tata Inst. Fund. Res. Lectures on Math. and Phys. 29, Tata Institute of Fundamental Research, Bombay, 1983. MR 85g:11034 Zbl 0539.10021

[Manin 1972] Y. I. Manin, "Parabolic points and zeta functions of modular curves", Izv. Akad. Nauk SSSR Ser. Mat. 36 (1972), 19-66. In Russian; translated in Math. USSR-Izv. 6 (1972), 19-64. MR 47 \#3396 Zbl 0243.14008

[Petersson 1948] H. Petersson, "Über den Bereich absoluter Konvergenz der Poincaréschen Reihen", Acta Math. 80 (1948), 23-63. MR 10,111d Zbl 0031.12502

[Petridis and Risager 2004] Y. N. Petridis and M. S. Risager, "Modular symbols have a normal distribution”, Geom. Funct. Anal. 14:5 (2004), 1013-1043. MR 2005h:11101 Zbl 1142.11332

[Roelcke 1966] W. Roelcke, "Das Eigenwertproblem der automorphen Formen in der hyperbolischen Ebene, I”, Math. Ann. 167:4 (1966), 292-337. MR 0243062 Zbl 0152.07705

[Roelcke 1967] W. Roelcke, "Das Eigenwertproblem der automorphen Formen in der hyperbolischen Ebene, II”, Math. Ann. 168 (1967), 261-324. MR 0243062 Zbl 0152.07705

[Slater 1960] L. J. Slater, Confluent hypergeometric functions, Cambridge University Press, New York, 1960. MR 21 \#5753 Zbl 0086.27502

[Sreekantan 2009] R. Sreekantan, "Higher order modular forms and mixed Hodge theory", Acta Arith. 139:4 (2009), 321-340. MR 2010j:11068 Zbl 1225.11052

Communicated by Peter Sarnak

Received 2011-04-29 Revised 2011-09-20 Accepted 2011-10-27

r.w.bruggeman@uu.nl Mathematisch Instituut, Universiteit Utrecht, Postbus 80010, 3508 TA Utrecht, Netherlands

nikolaos.diamantis@maths.nottingham.ac.uk

School of Mathematical Science, University of Nottingham,

Nottingham, NG7 2RD, United Kingdom 


\section{Algebra \& Number Theory}

msp.berkeley.edu/ant

\section{EDITORS}

MANAGING EDITOR

Bjorn Poonen

Massachusetts Institute of Technology

Cambridge, USA

\author{
EDITORIAL BOARD CHAIR \\ David Eisenbud \\ University of California \\ Berkeley, USA
}

\section{BOARD OF EDITORS}

Georgia Benkart

Dave Benson

Richard E. Borcherds

John H. Coates

J-L. Colliot-Thélène

Brian D. Conrad

Hélène Esnault

Hubert Flenner

Edward Frenkel

Andrew Granville

Joseph Gubeladze

Ehud Hrushovski

Craig Huneke

Mikhail Kapranov

Yujiro Kawamata

János Kollár

Yuri Manin

Barry Mazur

Philippe Michel
University of Wisconsin, Madison, USA

University of Aberdeen, Scotland

University of California, Berkeley, USA

University of Cambridge, UK

CNRS, Université Paris-Sud, France

University of Michigan, USA

Freie Universität Berlin, Germany

Ruhr-Universität, Germany

University of California, Berkeley, USA

Université de Montréal, Canada

San Francisco State University, USA

Hebrew University, Israel

University of Virginia, USA

Yale University, USA

University of Tokyo, Japan

Princeton University, USA

Northwestern University, USA

Harvard University, USA

École Polytechnique Fédérale de Lausanne
Susan Montgomery

Shigefumi Mori

Raman Parimala

Jonathan Pila

Victor Reiner

Karl Rubin

Peter Sarnak

Joseph H. Silverman

Michael Singer

Vasudevan Srinivas

J. Toby Stafford

Bernd Sturmfels

Richard Taylor

Ravi Vakil

Michel van den Bergh

Marie-France Vignéras

Kei-Ichi Watanabe

Andrei Zelevinsky

Efim Zelmanov
University of Southern California, USA

RIMS, Kyoto University, Japan

Emory University, USA

University of Oxford, UK

University of Minnesota, USA

University of California, Irvine, USA

Princeton University, USA

Brown University, USA

North Carolina State University, USA

Tata Inst. of Fund. Research, India

University of Michigan, USA

University of California, Berkeley, USA

Harvard University, USA

Stanford University, USA

Hasselt University, Belgium

Université Paris VII, France

Nihon University, Japan

Northeastern University, USA

University of California, San Diego, USA

\section{PRODUCTION}

production@msp.org

Silvio Levy, Scientific Editor

See inside back cover or www.jant.org for submission instructions.

The subscription price for 2012 is US \$175/year for the electronic version, and \$275/year ( $\$ 40$ shipping outside the US) for print and electronic. Subscriptions, requests for back issues from the last three years and changes of subscribers address should be sent to Mathematical Sciences Publishers, Department of Mathematics, University of California, Berkeley, CA 94720-3840, USA.

Algebra \& Number Theory (ISSN 1937-0652) at Mathematical Sciences Publishers, Department of Mathematics, University of California, Berkeley, CA 94720-3840 is published continuously online. Periodical rate postage paid at Berkeley, CA 94704, and additional mailing offices.

ANT peer review and production are managed by EditFLOW ${ }^{\circledR}$ from Mathematical Sciences Publishers.

PUBLISHED BY

mathematical sciences publishers

http://msp.org/

A NON-PROFIT CORPORATION

Typeset in IATEX

Copyright ( 2012 by Mathematical Sciences Publishers 


\section{Algebra \& Number Theory}

\section{Volume $6 \quad$ No. $7 \quad 2012$}

On the rank of the fibers of rational elliptic surfaces

Cecília Salgado

Néron's pairing and relative algebraic equivalence

CÉDRIC PÉPIN

Free subalgebras of quotient rings of Ore extensions

JASON P. BELL and DANIEL ROGALSKI

Classes de cycles motiviques étales

1369

BRUNO KAHN

Higher-order Maass forms

1409

ROELOF BRUGGEMAN and NiKOLAOS DIAMANTIS

Log canonical thresholds, $F$-pure thresholds, and nonstandard extensions

Bhargav Bhatt, Daniel J. Hernández, Lance Edward Miller and Mircea MUSTAȚĂ

The semistable reduction problem for the space of morphisms on $\mathbb{P}^{n}$

ALON LEVY

Grothendieck's trace map for arithmetic surfaces via residues and higher adèles

MATTHEW MORROW

Crystalline extensions and the weight part of Serre's conjecture

Toby GeE, Tong LiU and DAVID SAVITT

Annihilating the cohomology of group schemes 